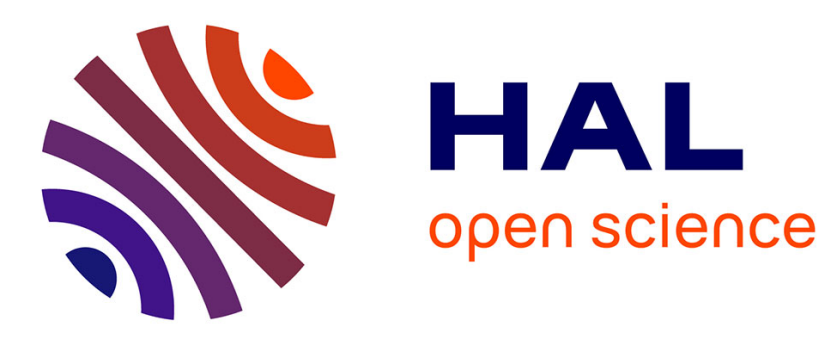

\title{
Miocene lateral extrusion in the inner western Alps revealed by dynamic fault analysis
}

Jean-Daniel Champagnac, Christian Sue, Bastien Delacou, Pierre Tricart, Cécile Allanic, Martin Burkhard

\section{- To cite this version:}

Jean-Daniel Champagnac, Christian Sue, Bastien Delacou, Pierre Tricart, Cécile Allanic, et al.. Miocene lateral extrusion in the inner western Alps revealed by dynamic fault analysis. Tectonics, 2006, 25, pp.TC3014. 10.1029/2004TC001779 . hal-00130576

\section{HAL Id: hal-00130576 https://hal.science/hal-00130576}

Submitted on 4 May 2021

HAL is a multi-disciplinary open access archive for the deposit and dissemination of scientific research documents, whether they are published or not. The documents may come from teaching and research institutions in France or abroad, or from public or private research centers.
L'archive ouverte pluridisciplinaire HAL, est destinée au dépôt et à la diffusion de documents scientifiques de niveau recherche, publiés ou non, émanant des établissements d'enseignement et de recherche français ou étrangers, des laboratoires publics ou privés. 


\title{
Miocene lateral extrusion in the inner western Alps revealed by dynamic fault analysis
}

\author{
J. D. Champagnac, ${ }^{1,2}$ C. Sue, ${ }^{1}$ B. Delacou, ${ }^{1}$ P. Tricart, ${ }^{3}$ C. Allanic, ${ }^{1}$ and M. Burkhard ${ }^{1}$
}

Received 16 December 2004; revised 2 February 2006; accepted 17 February 2006; published 8 June 2006.

[1] From early Miocene to the present-day the core parts of the western European Alps experienced brittle extensional deformations, mostly in a strike-parallel direction. Here we present new data constraining the brittle deformation of the Vanoise area (French Alps) and a synthesis of 312 paleostress tensors in the whole arc of the internal western Alps. The data show a continuous change in the direction of extension, from $\mathrm{N} 065^{\circ}$ (Simplon area), to N-S (Vanoise area) and to NNW-SSE (Briançon area). The abundance of orogenperpendicular $\sigma_{3}$ axes increases from the north to the south. In the Briançonnais area, an extensional reactivation of the Basal Penninic Thrust seems to be the origin of the E-W to NE-SW oriented $\sigma_{3}$. In light of these new data and the regional paleostress synthesis, we propose a predominant orogen-parallel extension in the internal zone as a whole. This orogen-parallel extension is related to the indentation/rotation of the Apulian microplate and to the opening of the Ligurian Sea during the lower-middle Miocene. The locally observed orogen-perpendicular extension is interpreted as an effect of the exhumation of the Internal Crystalline massifs, the uplift of the External Crystalline massifs and/or the present-day geodynamics (postorogenic gravitational collapse). Some transcurrent tectonics, older than the extension in the Valais area and younger than the extension farther south is observed in the entire inner western Alps; strike-slip movements are correlated with the Apulian rotation and local permutation of stress axes. Citation: Champagnac, J. D., C. Sue, B. Delacou, P. Tricart, C. Allanic, and M. Burkhard (2006), Miocene lateral extrusion in the inner western Alps revealed by dynamic fault analysis, Tectonics, 25, TC3014, doi:10.1029/2004TC001779.

\section{Introduction}

[2] The Alpine belt results from the subduction of the Tethyan and Valaisan ocean seafloors and the subsequent collision between the European and Apulian margin since

\footnotetext{
${ }^{1}$ Geological Institute, University of Neuchâtel, Neuchâtel, Switzerland.

${ }^{2}$ Now at Department of Geological Sciences and Cooperative Institute for Research in Environmental Science, University of Colorado, Boulder, Colorado, USA.

${ }^{3}$ Laboratoire de Géodynamique des Chaînes Alpines, Maison des Géosciences, Université de Grenoble, St Martin D’Hères, France.

Copyright 2006 by the American Geophysical Union. 0278-7407/06/2004TC001779
}

the upper Cretaceous [e.g., Tricart, 1984; Lemoine et al., 1986; Schmid and Kissling, 2000]. At the plate scale, Africa currently continues to converge with Europe at a rate of 3 $8 \mathrm{~mm} / \mathrm{yr}$ at the longitude of the western Alps [DeMets et al., 1994; Albarello et al., 1995; Nocquet and Calais, 2004]. Alpine tectonics have been studied in great detail, in terms of nappe emplacement history and geometry [e.g., Tricart, 1984; Escher and Beaumont, 1997; Pfiffner et al., 2000] as well as metamorphism and thermal evolution through time [Steck and Hunziker, 1994; Desmons et al., 1999; Brouwer et al., 2004]. Another important part of Alpine research focused on the arcuate shape of the belt and counterclockwise rotation of the Apulian promontory [e.g., Vialon et al., 1989; Collombet et al., 2002; Lickorish et al., 2002].

[3] In the past 10 years, emphasis has been placed on the study of late Alpine extensional tectonics under ductile to brittle conditions. Extension has been first described in the eastern Alps from the lower Miocene onward, with an E-W direction of extension, parallel to the Alpine trend [Selverstone, 1988; Ratschbacher et al., 1991; Frisch et al., 2000]. Ductile extension has also been locally observed in the central and western Alps [Ballève et al., 1990; Mancktelow, 1992; Steck and Hunziker, 1994; Rolland et al., 2000; Wawrzyniec et al., 2001; Tricart et al., 2004b]. In the western Alps, late Alpine brittle extension has been observed within inner parts of the belt [Aillères et al., 1995; Cannic et al., 1999; Sue and Tricart, 1999; Bistacchi et al., 2000; Champagnac et al., 2003, 2004; Grosjean et al., 2004; Tricart et al., 2004b]. Moreover, extension is currently active, as demonstrated by seismotectonics studies [Eva et al., 1998; Sue et al., 1999; Delacou et al., 2004; Kastrup et al., 2004, Delacou, 2005].

[4] In this paper we present a series of 66 new paleostress tensors from the Vanoise area (French Alps, Figure 1a), computed from fault striations measurements, and presented in section 2.2. This new data set fills an important gap in the large-scale paleostress map of the inner western Alps, which is now based on a total of 312 paleostress tensors (presented and analyzed in the section 3 ). These data provide tight constraints about the evolution of the prevailing principal stress axis directions $\sigma_{1}$ and $\sigma_{3}$ through time, from Miocene to Recent for the inner western Alps. It appears that paleostress axis directions deviate significantly from the current state of stress as revealed by earthquake focal plane mechanisms.

\section{Vanoise Area}

\subsection{Geological and Tectonic Setting}

[5] The study area, the so-called "massif de la Vanoise", is located in the western Alps (Figure 1a), between the 

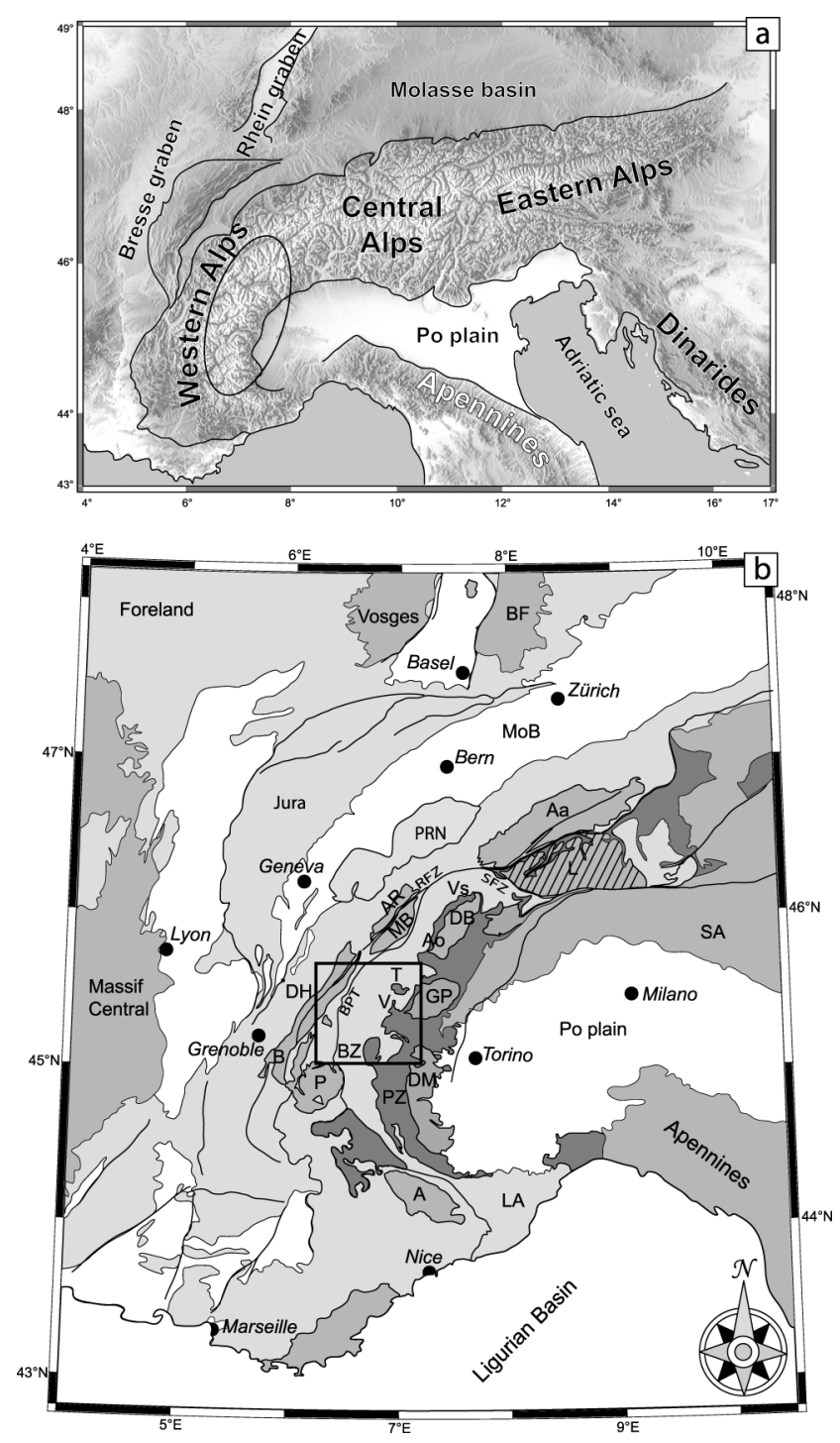

Figure 1. Location of the study area. (a) DEM (GTOPO30 database) of the Alps and surrounding areas. (b) Tectonic sketch map of the western and central Alps. A, Argentera; Aa, Aar; Ao, Aosta Valley; AR, Aiguilles Rouges; B, Belledonne; BPT, Basal Penninic Thrust; BR, Black forest; $\mathrm{BZ}$, Briançonnais zone; DB, Dent Blanche nappe; DH, Dauphiné/Helvetic zone; DM, Dora Maira; GP, Gran Paradiso; L, Lepontine dome; LA, Ligurian Alps; MoB, Molasse Bassin; MB, Mont Blanc; PZ, Piémont zone; P, Pelvoux; PRN, Prealpine Nappes; RFZ, Rhône Fault Zone; SA, southern Alps; SFZ, Simplon Fault Zone; T, Tarentaise valley; V, Vanoise; Vs, Valais.

Tarentaise and Maurienne valley (Figure 1b). The two valleys crosscut most of the geological units of the western Alps, namely, from bottom to top: continental units from the European margin (Belledonne external crystalline massif and corresponding Dauphinois and ultra-Dauphinois cover units), parts of the Briançonnais microcontinent, (including, Subbriançonnais cover units and the Gran Paradiso Internal
Crystalline massif), and, highest in the nappe pile, Piémont oceanic units (Cretaceous "Schistes-Lustrés" calcschists and ophiolites). The tectonometamorphic history of this area is highly complex, and includes polyphase Alpine folding and back folding under moderate to ultrahighpressure metamorphism from the Cretaceous to the Miocene [e.g., Desmons et al., 1999; Rolland et al., 2000].

[6] Extension under greenschist facies to brittle conditions has been described in the Upper Maurienne valley and attributed to the vertical indentation induced by the doming of the Gran Paradiso massif during convergence in front of the Apulian mantle buttress [Rolland et al., 2000]. Because of the large varieties of geological units, fault populations have been measured in numerous lithologies, especially schists, granites, and gneisses of the crystalline basement (Ambin, Gran Paradiso, and Briançonnais basement), quartzites, sandstones, and dolomites of thick Briançonnais Triassic series, and Cretaceous Piémont calcschists.

\subsection{Brittle Deformation}

\subsubsection{Field Observations}

[7] The brittle deformation observed in the field is mostly extensional: normal faults ranging in size from centimeters to hundreds of meters are most frequently observed, and no reverse faults have been identified. Locally, transcurrent faults are observed, as well as horizontal striations on normal fault planes (reactivation). Our field work is based on fault and striation measurements (quantitative analyses, see below) and a qualitative determination of the fault orientation, size and aspect. Figure 2 shows a multiscale analysis of a faulted area near the Iseran pass, between the Tarentaise and Maurienne valleys (site NEIGE, n41 and n42, and Table 1) in the Cretaceous Schistes Lustrés. In the panoramic view shown in Figure 2a, a large-scale normal fault is observed, together with several minor ones; the large fault dips to the SW and defines a reverse slope in the topography. Springs are found along the fault trace. Figure $2 \mathrm{~b}$ shows a large striated fault plane, SW dipping, and Figure 2c is a close-up showing two families of striations associated with this fault system. These observations and the measurement of about 40 couples of plane/striations at this site allow us to calculate two paleostress tensors with a reliable relative chronology (Figure 2d). From crosscutting relationships, the normal component clearly appears to be older than the transcurrent component. Note that the extensional axis $\left(\sigma_{3}\right)$ remains in the NNE-SSW orientation for both tensors (see Figure 2 caption for further details).

\subsubsection{Paleostress Computations}

[8] Field work includes the systematic measurement of fault populations, including fault plane orientation, striation orientation and shear sense, as well as a reliability criterion of the shear sense (certain, probable, uncertain). Resolved shear stress orientation on each fault surface is assumed to be parallel to the measured striation with the correct shear sense [Wallace, 1951; Bott, 1959]. We used a systematic collection of minor faults to determine paleostress axis direction using the "direct inversion method" of Angelier [1990]. About 1500 faults planes and their slickenlines were 

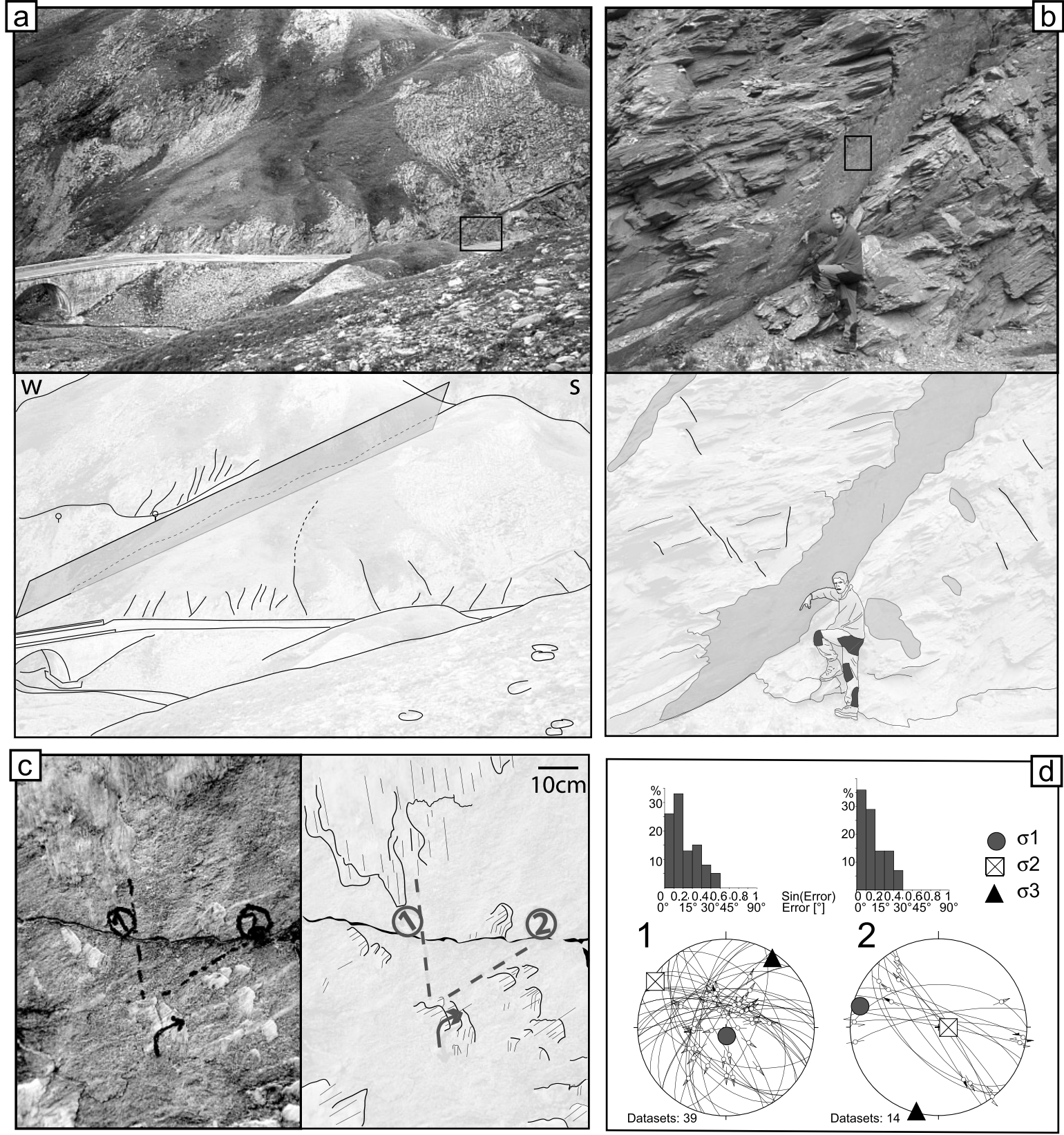

Figure 2. Fault analysis between Maurienne and Tarentaise valleys (site NEIGE N159 $/ \mathrm{N} 160^{\circ}$, close to the Iseran pass) with field views and their interpretations. (a) Morphological expression of a large normal fault viewed from the NE. The large west dipping fault is associated with smaller ones, east or west dipping. The bridge gives the scale. (b) Outcrop-scale view of one the minor fault (box in Figure 2a). The Cretaceous Schistes Lustrés limestones in the vicinity of the fault surface are broken into fault breccia. The geologist shows one of the striations of the fault surface. (c) Close-up of the same fault surface (box in Figure 2b) showing two directions of calcite slickenfibers; both directions have been drawn directly on the fault plane. Chronologically, the first direction is a downdip (normal fault), and the second one is subhorizontal (left lateral). (d) Measurement of several fault planes in this sector allows calculation of two paleostress tensors with a reliable relative chronology. For the computations and graphic representation of our data we used the "Tectonics FP" software [Sperner et al., 1993]. Plot of the calculated main stress axes (circle for $\sigma_{1}$, square for $\sigma_{2}$, and triangle for $\sigma_{3}$ ) are represented with fault planes and striations in lower hemisphere Schmidt stereograms. Also provided is the associated histogram of the misfit angle. The first tensor (extensional) and the second one (transcurrent) have the same $\sigma_{3}$ axis orientation. Furthermore, curved slickenfibers demonstrate a progressive transition between extensive and transcurrent movements. 
measured at 58 sites covering the entire Vanoise massif and the Maurienne valley. Results include the orientation of major, intermediate and minor stress axes $\left(\sigma_{1}, \sigma_{2}\right.$, and $\sigma_{3}$, respectively), the ellipsoid form parameter $\Phi=\left(\sigma_{2}-\sigma_{3}\right) /$ $\left(\sigma_{1}-\sigma_{3}\right)$ and the average misfit angle $\alpha$ (the angle between the computed optimum slickenline and the measured one). We estimated the quality of our paleotress tensors by way of several criteria:

[9] 1. The number of faults used in the calculation, their spatial coverage and the accuracy of the slickenline measurements. Sites with less than 10 faults were not considered. We also discarded some $10 \%$ of faults from our initial database.

[10] 2. The coherence between the inversion and the results from the geometrical solution (right dihedra method after Angelier [1979]).

[11] 3. The average misfit angle $\alpha$, which should be as low as possible, and its histogram repartition. The histogram should show a narrow half Gaussian distribution.

[12] 4. The visualization of the inverse function with Ginko software [Yamaji, 2000] to determine the stability of the inversion.

[13] The quality of stress tensors was classified from 1 (excellent) to 3 (low quality). According to this ranking system, 20 tensors $(30 \%)$ are of low quality, 33 tensors $(50 \%)$ are of medium quality while 13 tensors $(20 \%)$ are excellent. Note that 5 tensors (n24, n31, n35, n51, and n65) have been calculated from fault populations without striations and classified with the lower quality criterion. In these cases, the most likely slip direction was deduced from drag folds and "en echelon" tension gashes, or simply by assuming a dip slip movement in the case of conjugate sets of normal faults. In several sites, two crosscutting sets of slickenline families have been observed, allowing us to determine two paleostress tensor (e.g., Figure 2).

\subsubsection{Data Analysis}

[14] Geometrical results (fault planes and striations, and paleostress tensors) of the 66 new paleostress tensors are shown in Figure 3. Numerical results of the inversions are integrated to the global database (Table 1), numbered N119 (n1 in Figures 3 and 4) to N184 (n66 in Figures 3 and 4). In map form (Figure 4), the coverage of our paleostress tensor data set is concentrated in the internal zones of the western Alps, except for tensors n17, n34, n63, n34, n51, and n57, located between the External Crystalline massif (ECM) of Belledonne and the Basal Penninic Thrust (BPT). The arrows represent the projected $\sigma_{3}$ axes onto the horizontal plane for both transcurrent (white arrows) and extensional (black arrows) paleostress tensors. A first overview reveals a N-S to NNE-SSW general direction of $\sigma_{3}$ axes, as highlighted with the rose diagram on the upper right part of the map. Seventy-one percent (51 out of 66) of our tensors are extensional (i.e., $\sigma_{1}$ steep, $\sigma_{3}$ subhorizontal) and the other $29 \%$ (15 out of 66 ) are transcurrent (i.e., $\sigma_{2}$ steep, $\sigma_{1}$ subhorizontal). On the basis of crosscutting relationships, some local chronologies have been observed: for most of the studied sites, transcurrent movements are younger than extensional ones (e.g., Figure 2). This relative chronology is obvious in the Mont Cenis area (tensors n9 to n16), and consistent with the chronology all along the left side of the Maurienne valley, where conjugate normal faults bear clear evidence for a more recent reactivation with horizontal striations.

[15] The directions of principal stress axes are represented with rose diagrams (circular histograms, Figure 5a), with azimuth (Figure 5a, top) and plunge (Figure 5a, bottom) of axes. The majority of $\sigma_{1}$ axes are steep, with some random scatter around the vertical axis. A minor part of the population of $\sigma_{1}$ axes is subhorizontal, and related to transcurrent paleostress tensors; the $\sigma_{2}$ orientation is either subhorizontal (extensional stress state) or subvertical (transcurrent stress state), without any main direction. The most frequently observed direction of $\sigma_{3}$ axes is N-S to NNE$\mathrm{SSW}$, with some exceptions in an E-W orientation. All $\sigma_{3}$ axes are subhorizontal; this means that we did not calculated any compressional paleostress orientations. The plot of the entire data set of $\sigma_{1}$ and $\sigma_{3}$ axes on a Schmidt stereonet shows the spatial distribution of the stress axes (Figure 5b). The $\sigma_{1}$ axes (small circles) are predominantly in a nearvertical position (except for the transcurrent tensors), in a gently diffuse shape. The $\sigma_{3}$ axes (black triangles) are positioned horizontally. The contouring of $\sigma_{3}$ axes for the extensional and transcurrent states of stresses (Figure 5c) indicates coaxial direction for both transcurrent and extensional paleostress tensors. The global data set has also been used to calculate an "overall average" tensor for the entire area; we used all of the measured faults, even the fault planes discarded during stress inversion (but not the fault planes without lineation). The whole data set is composed of 1543 faults and their lineations. We applied the "right dihedra" (RD) method [Angelier, 1979] as well as "numerical-dynamical analysis" (NDA) [Sperner et al., 1993] to the entire data set. Because of the methods used and the size of the considered area, this calculation could be considered as a mean strain tensor for the entire region. The results are very similar and agree with the mean direction of extension determined by paleostress inversion: the minimum strain axis (the direction of extension) is oriented $021^{\circ} / 00^{\circ}$ (RD) and $198^{\circ} / 01^{\circ}$ (NDA). We also performed the same calculation for normal faults (i.e., with less than $45^{\circ}$ between fault dip and lineation plunge, $86 \%$ of the data set) and for transcurrent faults (14\% of the data set). Extensional axes are oriented with a very good correlation, $027^{\circ} / 00^{\circ}$ (RD) and $200^{\circ} / 01^{\circ}$ (NDA) for transcurrent faults, and $193^{\circ} / 07^{\circ}$ (RD) and $196^{\circ} / 04^{\circ}$ (NDA) for normal faults (Figure 5d). These strain analyses confirm the highly coaxial orientation of extensional axes for extensional stress/strain state and transcurrent stress/strain state. Despite local variations of orientations, the overall strain/stress state appears to be very stable, with a NNE-SSW direction of minimum stress/strain axis, for both transcurrent and extensional tensors.

[16] The analysis of the dip of fault planes and their associated striations (Figure 5e) demonstrates the predominance of dip slip normal faults: $50 \%$ of our data set is characterized by donwdip slip vectors, with less than $10^{\circ}$ difference between the plunge of the striation and the dip of the fault plane. The very low number of faults with a high angle between fault plunge and lineation plunge is taken as 
Table 1. Database of the 312 Paleostress Tensors Synthesized in This Paper ${ }^{\mathrm{a}}$

\begin{tabular}{|c|c|c|c|c|c|c|c|c|c|c|c|c|c|c|c|c|c|}
\hline \multicolumn{3}{|c|}{ Name } & \multicolumn{5}{|c|}{ Location } & \multicolumn{2}{|c|}{$\sigma_{1}$} & \multicolumn{2}{|c|}{$\sigma_{2}$} & \multicolumn{2}{|c|}{$\sigma_{3}$} & \multicolumn{4}{|c|}{ Parameters } \\
\hline $\mathrm{N}$ & $\mathrm{n}$ & Site & Lon & Lat & $\rho$ & $\tau$ & $\gamma$ & $\mathrm{az}$ & Dip & $\mathrm{az}$ & Dip & $\mathrm{az}$ & Dip & Data & $\Phi$ & $\alpha$ & Conf \\
\hline 1 & 1 & anniv (1) & 7.5641 & 46.2645 & 184166 & 56.87 & 62.9 & 271 & 71 & 82 & 17 & 174 & 9 & 12 & 0.52 & 6 & 1 \\
\hline 2 & 2 & anniv (2) & 7.5641 & 46.2645 & 184166 & 56.87 & 58.9 & 283 & 37 & 68 & 47 & 178 & 18 & 10 & 0.49 & 5.7 & 3 \\
\hline 3 & 3 & artsin & 7.4279 & 46.1166 & 177390 & 51.07 & 61.9 & 8 & 61 & 207 & 27 & 113 & 8 & 18 & 0.47 & 11.5 & 1 \\
\hline 4 & 4 & cargn & 7.5589 & 46.2326 & 181472 & 56.13 & 29.1 & 285 & 73 & 118 & 17 & 27 & 4 & 18 & 0.08 & 6.5 & 2 \\
\hline 5 & 5 & chevre & 7.4318 & 46.0181 & 168956 & 48.75 & 78.2 & 214 & 69 & 38 & 21 & 307 & 1 & 11 & 0.2 & 5.5 & 2 \\
\hline 6 & 6 & cleus1 & 7.3219 & 46.1148 & 182626 & 49.06 & 29.9 & 315 & 69 & 172 & 17 & 79 & 12 & 12 & 0.06 & 2.9 & 2 \\
\hline 7 & 7 & cleus2 & 7.327 & 46.1094 & 181913 & 49.02 & 18.0 & 355 & 53 & 148 & 33 & 247 & 13 & 11 & 0.32 & 3.2 & 1 \\
\hline 8 & 8 & couta & 7.4913 & 46.0824 & 171335 & 51.50 & 12.5 & 26 & 87 & 154 & 2 & 244 & 2 & 22 & 0.22 & 9.4 & 1 \\
\hline 9 & 9 & danger & 7.3227 & 46.0212 & 246457 & 31.64 & 5.6 & 320 & 48 & 101 & 35 & 206 & 20 & 16 & 0.95 & 6.7 & 1 \\
\hline 10 & 10 & dix & 7.4073 & 46.0887 & 176077 & 50.02 & 41.0 & 182 & 9 & 69 & 67 & 271 & 21 & 33 & 0.97 & 7.3 & 2 \\
\hline 11 & 11 & emdd (1) & 7.8388 & 46.223 & 169136 & 61.94 & 21.9 & 210 & 71 & 309 & 3 & 40 & 19 & 22 & 0.06 & 4.8 & 2 \\
\hline 12 & 12 & emdd (2) & 7.8388 & 46.223 & 169136 & 61.94 & 80.9 & 250 & 12 & 88 & 78 & 341 & 3 & 13 & 0.24 & 7.8 & 3 \\
\hline 13 & 13 & emmd2 & 7.8609 & 46.2319 & 169192 & 62.61 & 29.6 & 202 & 81 & 303 & 2 & 33 & 8 & 16 & 0.07 & 7.6 & 1 \\
\hline 14 & 14 & ergil & 7.7082 & 46.2959 & 181165 & 60.40 & 18.4 & 250 & 74 & 134 & 7 & 42 & 14 & 11 & 0.4 & 5.3 & 2 \\
\hline 15 & 15 & ergi2 & 7.7062 & 46.2898 & 180655 & 60.25 & 37.8 & 226 & 64 & 2 & 19 & 98 & 17 & 9 & 0.93 & 9.8 & 3 \\
\hline 16 & 16 & evole & 7.5043 & 46.1085 & 172950 & 52.37 & 0.6 & 2 & 53 & 131 & 25 & 233 & 25 & 28 & 0.26 & 4.3 & 1 \\
\hline 17 & 17 & findel & 7.7856 & 46.0126 & 151398 & 56.30 & 19.7 & 179 & 36 & 326 & 49 & 76 & 17 & 21 & 0.77 & 9.7 & 2 \\
\hline 18 & 18 & forcl & 7.4563 & 46.0302 & 168689 & 49.54 & 30.5 & 20 & 64 & 164 & 21 & 260 & 14 & 15 & 0.45 & 7.7 & 1 \\
\hline 19 & 19 & furi (1) & 7.7326 & 45.9992 & 152567 & 54.71 & 56.3 & 178 & 63 & 26 & 25 & 291 & 11 & 21 & 0.08 & 6.3 & 1 \\
\hline 20 & 20 & furi (2) & 7.7326 & 45.9992 & 152567 & 54.71 & 40.3 & 5 & 1 & 107 & 83 & 275 & 7 & 9 & 0.91 & 5 & 2 \\
\hline 21 & 21 & gallen & 7.7891 & 46.0486 & 154558 & 57.22 & 22.2 & 53 & 59 & 309 & 8 & 215 & 30 & 9 & 0.79 & 4.3 & 2 \\
\hline 22 & 22 & gorner & 7.7867 & 45.9847 & 148807 & 55.65 & 79.4 & 130 & 75 & 227 & 2 & 315 & 15 & 16 & 0.04 & 10 & 2 \\
\hline 23 & 23 & grime & 7.5604 & 46.1609 & 174930 & 54.67 & 14.3 & 129 & 82 & 340 & 7 & 249 & 4 & 29 & 0.32 & 8.6 & 1 \\
\hline 24 & 24 & ires & 7.2418 & 46.0733 & 183526 & 46.66 & 75.7 & 261 & 49 & 48 & 36 & 151 & 17 & 9 & 0.24 & 7.6 & 3 \\
\hline 25 & 25 & leuker & 7.6603 & 46.3134 & 184732 & 59.72 & 86.3 & 129 & 58 & 344 & 27 & 146 & 16 & 18 & 0.23 & 7 & 2 \\
\hline 26 & 26 & moiry (1) & 7.5769 & 46.1122 & 169823 & 53.94 & 14.1 & 2 & 32 & 124 & 40 & 248 & 34 & 28 & 0.45 & 10.3 & 1 \\
\hline 27 & 27 & moiry (2) & 7.5769 & 46.1122 & 169823 & 53.94 & 45.9 & 277 & 3 & 151 & 84 & 8 & 4 & 24 & 0.63 & 16.5 & 3 \\
\hline 28 & 28 & monv1 & 7.3596 & 45.9852 & 170160 & 46.53 & 69.5 & 48 & 69 & 204 & 19 & 296 & 8 & 13 & 0.11 & 6.3 & 3 \\
\hline 29 & 29 & monv2 & 7.3415 & 46.005 & 172711 & 46.71 & 17.3 & 334 & 13 & 196 & 72 & 64 & 11 & 25 & 0.49 & 9.6 & 1 \\
\hline 30 & 30 & $\operatorname{nax}$ & 7.4597 & 46.2412 & 186725 & 54.38 & 6.6 & 318 & 82 & 151 & 8 & 61 & 2 & 9 & 0.22 & 6.1 & 3 \\
\hline 31 & 31 & nikla & 7.7879 & 46.1638 & 165410 & 59.65 & 3.4 & 152 & 5 & 34 & 79 & 243 & 10 & 22 & 0.49 & 12.5 & 2 \\
\hline 32 & 32 & randa1 & 7.7751 & 46.0878 & 158787 & 57.76 & 77.8 & 105 & 59 & 241 & 24 & 340 & 19 & 13 & 0.78 & 13.6 & 3 \\
\hline 33 & 33 & randa2 & 7.7615 & 46.0847 & 159077 & 57.38 & 40.4 & 107 & 73 & 287 & 17 & 17 & 0 & 27 & 0.13 & 7.5 & 1 \\
\hline 34 & 34 & rech1 (1) & 7.4992 & 46.2461 & 185361 & 55.24 & 62.8 & 22 & 23 & 326 & 62 & 118 & 14 & 9 & 0.78 & 2 & 2 \\
\hline 35 & 35 & rech1 (2) & 7.4992 & 46.2461 & 185361 & 55.24 & 89.8 & 209 & 68 & 58 & 19 & 325 & 10 & 13 & 0.13 & 5 & 2 \\
\hline 36 & 36 & rech2 & 7.4953 & 46.2416 & 185132 & 55.07 & 48.1 & 173 & 76 & 276 & 3 & 7 & 14 & 19 & 0.03 & 8.9 & 1 \\
\hline 37 & 37 & rotba & 7.8215 & 46.0656 & 154772 & 58.38 & 3.6 & 350 & 46 & 138 & 39 & 242 & 16 & 14 & 0.04 & 2.4 & 2 \\
\hline 38 & 38 & roten & 7.7674 & 45.9866 & 149845 & 55.22 & 68.2 & 258 & 8 & 19 & 75 & 167 & 12 & 12 & 0.75 & 5.5 & 2 \\
\hline 39 & 39 & sarray & 7.2593 & 46.0606 & 181582 & 46.65 & 20.3 & 353 & 51 & 148 & 37 & 247 & 12 & 16 & 0.87 & 4.8 & 1 \\
\hline 40 & 40 & schler & 7.2715 & 46.0697 & 181636 & 47.09 & 2.1 & 239 & 65 & 137 & 5 & 45 & 24 & 8 & 0.16 & 3.8 & 3 \\
\hline 41 & 41 & solay1 & 7.5481 & 46.0653 & 167081 & 52.25 & 14.8 & 329 & 22 & 195 & 60 & 67 & 19 & 8 & 0.06 & 6.2 & 3 \\
\hline 42 & 42 & solay2 & 7.5385 & 46.0743 & 168330 & 52.26 & 24.7 & 150 & 71 & 349 & 18 & 257 & 6 & 16 & 0.17 & 8.6 & 2 \\
\hline 43 & 43 & solay3 & 7.534 & 46.0761 & 168705 & 52.21 & 57.8 & 0 & 50 & 212 & 36 & 110 & 16 & 11 & 0.98 & 3.5 & 3 \\
\hline 44 & 44 & stlu1 & 7.5939 & 46.2312 & 179813 & 56.81 & 8.2 & 178 & 67 & 331 & 21 & 65 & 10 & 13 & 0.23 & 3.3 & 1 \\
\hline 45 & 45 & stlu2 & 7.6029 & 46.2182 & 178222 & 56.73 & 12.3 & 151 & 37 & 353 & 51 & 249 & 11 & 15 & 0.6 & 6.3 & 2 \\
\hline 46 & 46 & tdela & 7.3743 & 46.1571 & 183602 & 50.94 & 56.9 & 238 & 61 & 91 & 25 & 354 & 14 & 16 & 0.08 & 3.9 & 3 \\
\hline 47 & 47 & thyon & 7.3709 & 46.1787 & 185505 & 51.40 & 2.4 & 262 & 70 & 142 & 10 & 49 & 17 & 14 & 0.29 & 6.3 & 2 \\
\hline 48 & 48 & trift & 7.4505 & 46.0245 & 154479 & 55.51 & 37.5 & 123 & 81 & 288 & 9 & 18 & 2 & 35 & 0.33 & 8.7 & 1 \\
\hline 49 & 49 & turgl & 7.7096 & 46.1496 & 167301 & 57.60 & 27.4 & 49 & 89 & 175 & 1 & 265 & 1 & 15 & 0.83 & 10.4 & 2 \\
\hline 50 & 50 & turtm & 7.6913 & 46.1714 & 170108 & 57.65 & 9.3 & 22 & 57 & 147 & 21 & 247 & 25 & 27 & 0.24 & 7.5 & 1 \\
\hline 51 & 51 & verc1 & 7.4558 & 46.2582 & 184385 & 56.39 & 36.4 & 147 & 73 & 287 & 13 & 20 & 11 & 19 & 0.01 & 11.4 & 2 \\
\hline 52 & 52 & verc2 & 7.5433 & 46.2479 & 183553 & 56.13 & 3.1 & 122 & 73 & 325 & 16 & 233 & 6 & 18 & 0.3 & 10.6 & 2 \\
\hline 53 & 53 & veyso & 7.3321 & 46.194 & 188733 & 51.03 & 2.0 & 118 & 65 & 323 & 23 & 229 & 9 & 9 & 0.14 & 3.8 & 2 \\
\hline 54 & 54 & vingt & 7.3956 & 46.0752 & 175547 & 49.48 & 33.5 & 271 & 81 & 173 & 1 & 83 & 9 & 16 & 0.57 & 3.8 & 1 \\
\hline 55 & 55 & visso & 7.5809 & 46.2344 & 180668 & 56.61 & 61.4 & 205 & 8 & 91 & 71 & 298 & 17 & 17 & 0.85 & 5.6 & 3 \\
\hline 56 & 56 & zeneg & 7.871 & 46.2795 & 173517 & 63.66 & 37.7 & 300 & 19 & 82 & 67 & 206 & 13 & 20 & 0.59 & 6.2 & 3 \\
\hline 57 & 57 & AVISE & 7.1526 & 45.7059 & 162847 & 34.75 & 9.3 & 5 & 63 & 128 & 16 & 224 & 22 & 17 & 0.05 & 7.3 & 1 \\
\hline 58 & 58 & BAUCHE & 6.7464 & 45.5394 & 182164 & 24.36 & 63.6 & 9 & 75 & 177 & 15 & 268 & 3 & 18 & 0.62 & 5.9 & 1 \\
\hline 59 & 59 & BISELX & 7.2227 & 46.0058 & 179337 & 44.64 & 62.4 & 218 & 58 & 107 & 13 & 107 & 13 & 16 & 0.99 & 2.8 & 2 \\
\hline 60 & 60 & BUTHIER & 7.2713 & 45.7845 & 160510 & 39.21 & 26.2 & 273 & 49 & 111 & 40 & 13 & 9 & 16 & 0.323 & 5.1 & 1 \\
\hline 61 & 61 & CARLO & 7.003 & 45.7136 & 173143 & 32.86 & 29.1 & 50 & 65 & 150 & 5 & 242 & 24 & 20 & 0.89 & 8.1 & 1 \\
\hline 62 & 62 & CHAP & 6.7318 & 45.6937 & 190460 & 28.99 & 58.0 & 343 & 8 & 249 & 30 & 87 & 59 & 16 & 0.36 & 10.1 & 2 \\
\hline 63 & 63 & CHAT1 & 6.858 & 45.6178 & 177868 & 28.03 & 40.0 & 183 & 72 & 79 & 5 & 348 & 17 & 15 & 0.264 & 7.8 & 3 \\
\hline 64 & 64 & СНАТ2 & 6.8628 & 45.4148 & 177384 & 27.99 & 1.0 & 218 & 61 & 119 & 5 & 27 & 28 & 18 & 0.008 & 1.8 & 1 \\
\hline 65 & 65 & ECHEV & 7.2638 & 45.8026 & 162140 & 39.61 & 76.4 & 31 & 61 & 205 & 29 & 296 & 2 & 28 & 0.518 & 4 & 1 \\
\hline 66 & 66 & FERRE & 7.1212 & 45.911 & 178314 & 40.44 & 19.4 & 273 & 28 & 149 & 47 & 21 & 30 & 12 & 0.06 & \# & 3 \\
\hline 67 & 67 & FRETE (1) & 6.8085 & 45.5709 & 179118 & 25.99 & 55.0 & 268 & 55 & 51 & 29 & 151 & 17 & 17 & 0.068 & 4 & 1 \\
\hline
\end{tabular}


Table 1. (continued)

\begin{tabular}{|c|c|c|c|c|c|c|c|c|c|c|c|c|c|c|c|c|c|}
\hline \multicolumn{3}{|c|}{ Name } & \multicolumn{5}{|c|}{ Location } & \multicolumn{2}{|c|}{$\sigma_{1}$} & \multicolumn{2}{|c|}{$\sigma_{2}$} & \multicolumn{2}{|c|}{$\sigma_{3}$} & \multicolumn{4}{|c|}{ Parameters } \\
\hline $\mathrm{N}$ & $\mathrm{n}$ & Site & Lon & Lat & $\rho$ & $\tau$ & $\gamma$ & $\mathrm{az}$ & Dip & az & Dip & $\mathrm{az}$ & Dip & Data & $\Phi$ & $\alpha$ & Conf \\
\hline 68 & 68 & FRETE (2) & 6.8085 & 45.5709 & 179118 & 25.99 & 4.0 & 291 & 8 & 147 & 80 & 22 & 6 & 8 & 0.565 & 10.2 & 3 \\
\hline 69 & 69 & GSB1 & 7.1877 & 45.8865 & 172592 & 40.82 & 33.8 & 101 & 11 & 215 & 65 & 7 & 22 & 21 & 0.91 & 7.7 & 3 \\
\hline 70 & 70 & GSB2 & 7.1896 & 45.8995 & 173407 & 41.22 & 5.8 & 313 & 24 & 159 & 64 & 47 & 10 & 19 & 0.577 & 16.9 & 2 \\
\hline 71 & 71 & GSBIT & 7.1512 & 45.8598 & 172949 & 39.46 & 21.5 & 347 & 25 & 108 & 48 & 241 & 32 & 15 & 0.37 & 4.6 & 2 \\
\hline 72 & 72 & GURRAZ & 6.9033 & 45.6249 & 175081 & 28.78 & 25.2 & 359 & 75 & 142 & 12 & 234 & 9 & 24 & 0.234 & 6.1 & 1 \\
\hline 73 & 73 & ISERAN & 7.021 & 45.4317 & 157752 & 23.38 & 58.6 & 84 & 84 & 352 & 0 & 262 & 6 & 34 & 0.2 & 9.4 & 1 \\
\hline 74 & 74 & LACPLAG & 6.845 & 45.488 & 172856 & 23.60 & 27.4 & 319 & 65 & 141 & 25 & 51 & 1 & 14 & 0.07 & 3.7 & 2 \\
\hline 75 & 75 & MALAT & 7.1313 & 45.8409 & 172881 & 38.60 & 25.6 & 271 & 32 & 129 & 52 & 13 & 19 & 15 & 0.18 & 4.7 & 2 \\
\hline 76 & 76 & MALAT2 & 7.1113 & 45.8408 & 174114 & 38.29 & 30.3 & 275 & 21 & 117 & 67 & 8 & 8 & 14 & 0.252 & 4.3 & 2 \\
\hline 77 & 77 & MARTI & 7.0814 & 46.0918 & 193850 & 44.48 & 73.5 & 325 & 22 & 231 & 10 & 118 & 65 & 11 & 0.576 & 9.7 & 3 \\
\hline 78 & 78 & MICOEUR & 7.2449 & 46.1183 & 186956 & 47.79 & 14.8 & 136 & 73 & 302 & 16 & 33 & 4 & 10 & 0.55 & 6.8 & 3 \\
\hline 79 & 79 & MONAL (1) & 6.9009 & 45.5687 & 172471 & 26.91 & 48.1 & 48 & 85 & 165 & 2 & 255 & 4 & 20 & 0.25 & 11.8 & 1 \\
\hline 80 & 80 & MONAL (2) & 6.9009 & 45.5687 & 172471 & 26.91 & 2.1 & 119 & 36 & 298 & 54 & 29 & 0 & 11 & 0.539 & 6.2 & 2 \\
\hline 81 & 81 & NIORD & 7.2035 & 45.9589 & 176910 & 43.08 & 51.1 & 261 & 2 & 168 & 59 & 352 & 31 & 16 & 0.57 & 9.2 & 2 \\
\hline 82 & 82 & PEIPOU & 6.818 & 45.5253 & 176399 & 24.59 & 36.4 & 142 & 76 & 142 & 76 & 241 & 2 & 22 & 0.25 & 13 & 1 \\
\hline 83 & 83 & PLAN & 6.9258 & 45.6244 & 173501 & 29.03 & 24.0 & 284 & 16 & 39 & 55 & 185 & 30 & 14 & 0.603 & 9 & 2 \\
\hline 84 & 84 & RECUL & 6.9618 & 45.4669 & 163528 & 24.04 & 87.0 & 171 & 69 & 31 & 17 & 297 & 13 & 19 & 0.16 & 10.1 & 1 \\
\hline 85 & 85 & RIDD & 7.2233 & 46.1551 & 191076 & 48.27 & 50.3 & 119 & 68 & 264 & 19 & 358 & 12 & 22 & 0.815 & 8.8 & 2 \\
\hline 86 & 86 & SAPIN & 7.1486 & 46.1063 & 191226 & 45.89 & 17.1 & 352 & 73 & 151 & 16 & 243 & 6 & 15 & 0.77 & 7.8 & 2 \\
\hline 87 & 87 & SEIGNE & 6.8091 & 45.7528 & 188335 & 31.61 & 23.6 & 308 & 48 & 82 & 32 & 188 & 24 & 17 & 0.779 & 7.1 & 2 \\
\hline 88 & 88 & STFOY (1) & 6.9313 & 45.5787 & 170819 & 27.58 & 59.4 & 91 & 56 & 359 & 2 & 267 & 34 & 16 & 0.701 & 4 & 1 \\
\hline 89 & 89 & STFOY (2) & 6.9313 & 45.5787 & 170819 & 27.58 & 45.4 & 157 & 11 & 48 & 61 & 253 & 27 & 14 & 0.492 & 7.1 & 2 \\
\hline 90 & 90 & THUIL & 6.8991 & 45.7203 & 180467 & 31.73 & 36.7 & 88 & 71 & 265 & 19 & 355 & 1 & 10 & 0.53 & 4 & 3 \\
\hline 91 & 91 & TIGNES1 & 6.9199 & 45.5054 & 168218 & 24.97 & 15.0 & 32 & 73 & 129 & 2 & 220 & 17 & 17 & 0.127 & 5.1 & 1 \\
\hline 92 & 92 & TIGNES2 & 6.9254 & 45.4539 & 167310 & 24.62 & 89.6 & 195 & 25 & 60 & 56 & 295 & 21 & 13 & 0.418 & 7.3 & 2 \\
\hline 93 & 93 & TIGNES3 & 6.9442 & 45.4977 & 166841 & 24.84 & 16.8 & 65 & 75 & 280 & 12 & 188 & 8 & 11 & 0.812 & 4.3 & 2 \\
\hline 94 & 94 & TOULJE & 7.1882 & 45.922 & 174777 & 41.70 & 2.3 & 300 & 47 & 145 & 40 & 44 & 13 & 24 & 0.87 & 6.5 & 2 \\
\hline 95 & 95 & VALDER1 & 7.1055 & 45.54 & 156734 & 28.37 & 81.6 & 316 & 51 & 209 & 13 & 110 & 36 & 12 & 0.07 & 3.6 & 2 \\
\hline 96 & 96 & VALDER2 & 7.1119 & 45.546 & 157102 & 29.03 & 5.0 & 272 & 78 & 115 & 11 & 24 & 5 & 13 & 0.33 & 6.6 & 1 \\
\hline 97 & 97 & VALDER3 & 7.122 & 45.5882 & 158115 & 30.31 & 46.3 & 83 & 50 & 248 & 39 & 344 & 7 & 11 & 0.922 & 6.5 & 3 \\
\hline 98 & 98 & VALDER4 (1) & 7.0259 & 45.6242 & 158809 & 31.85 & 0.2 & 79 & 79 & 303 & 8 & 212 & 8 & 10 & 0.05 & 14.7 & 2 \\
\hline 99 & 99 & ALDER4 (2) & 7.0259 & 45.6242 & 158809 & 31.85 & 56.8 & 247 & 3 & 343 & 63 & 155 & 26 & 12 & 0.65 & 2.9 & 2 \\
\hline 100 & 100 & VALGR1 & 7.1562 & 45.6978 & 162121 & 34.54 & 36.5 & 36 & 73 & 271 & 10 & 178 & 14 & 12 & 0.09 & 4.1 & 2 \\
\hline 101 & 101 & VALGR25 & 7.0482 & 45.6041 & 164006 & 29.88 & 67.1 & 16 & 56 & 183 & 33 & 277 & 6 & 22 & 0.74 & 10.5 & 2 \\
\hline 102 & 102 & VAI GR 3 & 7.0632 & 45.6206 & 163870 & 30.63 & 5.4 & 228 & 87 & 126 & 1 & 36 & 3 & 12 & 05 & 5.1 & 2 \\
\hline 103 & 103 & LGR4 & 7.1008 & 45.679 & 1646 & 33.11 & 55.9 & 319 & 39 & 204 & 28 & 89 & 38 & 20 & 0.95 & 4.5 & 2 \\
\hline 104 & 104 & VALP1 (1) & 7.2974 & 45.8715 & 165025 & 42.25 & 73.7 & 26 & 5 & 168 & 84 & 296 & 4 & 17 & 0.76 & 8.8 & 2 \\
\hline 105 & 105 & VALP1 (2) & 7.2974 & 45.8715 & 165025 & 42.25 & 56.7 & 117 & 78 & 9 & 4 & 279 & 11 & 21 & 0.62 & 9.6 & 3 \\
\hline 106 & 106 & VALP2 (1) & 7.4924 & 45.9007 & 156241 & 46.80 & 2.2 & 161 & 57 & 312 & 30 & 49 & 13 & 12 & 0.345 & 11.4 & 2 \\
\hline 107 & 107 & $2(2)$ & 7.4924 & 45.9007 & 156241 & 46 & 36.8 & 98 & 31 & 188 & 59 & 190 & 4 & 11 & 0.949 & 5.1 & 3 \\
\hline 108 & 108 & LP3 & 7.4628 & 45.8881 & 156868 & 45.85 & 36.8 & 196 & 85 & 99 & 1 & 9 & 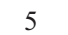 & 13 & 0.707 & 6.1 & 2 \\
\hline 109 & 109 & VALP4 (1) & 7.437 & 45.8782 & 157530 & 45.05 & 41.0 & 94 & 4 & 294 & 86 & 184 & 1 & 14 & 0.256 & 6.8 & 3 \\
\hline 110 & 110 & VALP4 (2) & 7.437 & 45.8782 & 157530 & 45.05 & 21.0 & 359 & 75 & 155 & 13 & 246 & 6 & 17 & 0.07 & 8 & 1 \\
\hline 111 & 111 & VALP5 & 7.3534 & 45.8323 & 161924 & 41.17 & 10.2 & 301 & 38 & 122 & 55 & 31 & 0 & 17 & 0.548 & 7 & 3 \\
\hline 112 & 112 & VALSA1 (1) & 7.2008 & 45.5295 & 149591 & 29.27 & 0. & 88 & 45 & 319 & 32 & 210 & 27 & 11 & 0.13 & 3 & 2 \\
\hline 113 & 113 & VALSA1 (2) & 7.2008 & 45.5295 & 149591 & 29.27 & 2.3 & 101 & 35 & 322 & 47 & 207 & 21 & 10 & 0.511 & 8.6 & 3 \\
\hline 114 & 114 & VALSA2 & 7.2115 & 45.5228 & 150088 & 30.29 & 2.3 & 91 & 36 & 327 & 38 & 208 & 32 & 11 & 0.48 & 4.1 & 3 \\
\hline 115 & 115 & VALSA3 & 7.2032 & 45.6525 & 156315 & 33.73 & 15.3 & 28 & 79 & 139 & 4 & 229 & 11 & 10 & 0.16 & 4.2 & 2 \\
\hline 116 & 116 & VAI SAM & 7.2051 & 45.6742 & 157494 & 34.50 & 3.5 & 93 & 70 & 304 & 17 & 211 & 10 & 16 & 0.773 & 14.4 & 2 \\
\hline 117 & 117 & LAN & 7.2591 & 45.9267 & 171307 & 43.15 & 13.1 & 300 & 1 & 201 & 85 & 30 & 5 & 16 & 0.499 & 8 & 2 \\
\hline 118 & 118 & VENS & 7.1241 & 46.0865 & 191048 & 45.03 & 34.0 & 278 & 8 & 165 & 70 & 11 & 19 & 26 & 0.635 & 19.9 & 3 \\
\hline 119 & 119 & AMBIN & 6.8738 & 45.1858 & 161203 & 12.79 & 16.8 & 67 & 70 & 269 & 19 & 176 & 7 & 75 & 0.12 & 10.5 & 1 \\
\hline 120 & 120 & ARPON1 & 6.6961 & 45.3295 & 178459 & 16.96 & 87.0 & 220 & 73 & 18 & 16 & 110 & 6 & 14 & 0.21 & 7.2 & 2 \\
\hline 121 & 121 & ARPON2 & 6.7123 & 45.3169 & 176865 & 16.63 & 20.4 & 131 & 59 & 306 & 31 & 37 & 2 & 38 & 0.55 & 9.3 & 2 \\
\hline 122 & 122 & $\mathrm{FE}, \mathrm{C}$ & 7.0884 & 45.2918 & 147746 & 18.57 & 64.4 & 335 & 75 & 174 & 14 & 83 & 5 & 20 & 0.49 & 9.3 & 1 \\
\hline 123 & 123 & AVERO2 & 7.0306 & 45.3236 & 153136 & 19.33 & 69.7 & 29 & 85 & 179 & 4 & 269 & 2 & 45 & 0.49 & 12.4 & 1 \\
\hline 124 & 124 & BARDO & 6.7172 & 45.0895 & 171680 & 8.49 & 2.5 & 237 & 75 & 103 & 10 & 11 & 10 & 31 & 0.06 & 13.5 & 1 \\
\hline 125 & 125 & BOZEL & 6.6236 & 45.4446 & 187619 & 20.27 & 25.7 & 135 & 53 & 317 & 37 & 226 & 1 & 31 & 0.55 & 14.9 & 2 \\
\hline 126 & 126 & CELS & 6.9431 & 45.1048 & 154282 & 9.91 & 3.1 & 302 & 33 & 72 & 45 & 193 & 27 & 14 & 0.51 & 10.9 & 3 \\
\hline 127 & 127 & CENIS1 & 6.9271 & 45.2269 & 158077 & 14.72 & 24.3 & 325 & 79 & 129 & 10 & 219 & 3 & 31 & 0.21 & 10.9 & 2 \\
\hline 128 & 128 & CENIS2(1) & 6.9614 & 45.2292 & 155519 & 15.03 & 18.0 & 264 & 68 & 87 & 22 & 357 & 1 & 17 & 0.23 & 11.2 & 1 \\
\hline 129 & 129 & CENIS2(2) & 6.9614 & 45.2292 & 155519 & 15.03 & 4.0 & 107 & 6 & 4 & 66 & 199 & 23 & 18 & 0.63 & 7.4 & 2 \\
\hline 130 & 130 & CENIS3 & 6.9243 & 45.2831 & 159833 & 16.88 & 13.1 & 314 & 65 & 107 & 22 & 210 & 10 & 16 & 0.67 & 8.8 & 2 \\
\hline 131 & 131 & CENIS4 & 6.9822 & 45.1886 & 152919 & 13.53 & 73.5 & 346 & 83 & 178 & 6 & 87 & 1 & 19 & 0.52 & 10.3 & 2 \\
\hline 132 & 132 & CENIS5 & 6.8634 & 45.2473 & 163465 & 15.09 & 55.9 & 333 & 76 & 161 & 14 & 71 & 2 & 17 & 0.37 & 8.2 & 2 \\
\hline 133 & 133 & CENIS6(1) & 6.9695 & 45.2137 & 154500 & 14.47 & 8.5 & 1 & 74 & 111 & 6 & 203 & 15 & 32 & 0.19 & 8.3 & 1 \\
\hline 134 & 134 & CENIS6(2) & 6.9695 & 45.2137 & 154500 & 14.47 & 76.5 & 181 & 11 & 337 & 78 & 91 & 5 & 29 & 0.92 & 6.6 & 2 \\
\hline
\end{tabular}


Table 1. (continued)

\begin{tabular}{|c|c|c|c|c|c|c|c|c|c|c|c|c|c|c|c|c|c|}
\hline \multicolumn{3}{|c|}{ Name } & \multicolumn{5}{|c|}{ Location } & \multicolumn{2}{|c|}{$\sigma_{1}$} & \multicolumn{2}{|c|}{$\sigma_{2}$} & \multicolumn{2}{|c|}{$\sigma_{3}$} & \multicolumn{4}{|c|}{ Parameters } \\
\hline $\mathrm{N}$ & $\mathrm{n}$ & Site & Lon & Lat & $\rho$ & $\tau$ & $\gamma$ & $\mathrm{az}$ & Dip & $\mathrm{az}$ & Dip & az & Dip & Data & $\Phi$ & $\alpha$ & Conf \\
\hline 135 & 135 & CHAMB & 6.2788 & 45.3873 & 211715 & 16.32 & 29.7 & 317 & 24 & 133 & 65 & 226 & 1 & 18 & 0.49 & 11.5 & 2 \\
\hline 136 & 136 & CHAMPA & 6.7087 & 45.4599 & 181892 & 21.44 & 11.4 & 278 & 7 & 171 & 68 & 10 & 21 & 26 & 0.35 & 11.7 & 2 \\
\hline 137 & 137 & CHAMPA2 & 6.7167 & 45.4566 & 181179 & 21.40 & 17.4 & 276 & 8 & 40 & 75 & 184 & 12 & 17 & 0.54 & 12.8 & 2 \\
\hline 138 & 138 & ECOT & 7.0927 & 45.3807 & 150651 & 22.20 & 66.8 & 340 & 66 & 183 & 22 & 89 & 8 & 23 & 0.16 & 4.2 & 2 \\
\hline 139 & 139 & ETACHE & 6.8136 & 45.1613 & 165347 & 11.55 & 2.5 & 9 & 73 & 104 & 1 & 194 & 16 & 35 & 0.05 & 4.7 & 2 \\
\hline 140 & 140 & EXTRA & 6.7986 & 45.2186 & 167718 & 13.62 & 19.4 & 145 & 66 & 299 & 22 & 33 & 10 & 10 & 0.26 & 3.5 & 3 \\
\hline 141 & 141 & FONDS & 6.6524 & 45.3089 & 181183 & 15.97 & 40.0 & 10 & 69 & 142 & 14 & 236 & 15 & 43 & 0.17 & 11.5 & 1 \\
\hline 142 & 142 & FONT & 6.5006 & 45.4514 & 197002 & 19.59 & 5.6 & 296 & 59 & 100 & 30 & 194 & 7 & 24 & \# & \# & 3 \\
\hline 143 & 143 & FOUR & 7.0167 & 45.3945 & 156765 & 21.95 & 24.0 & 101 & 78 & 318 & 10 & 226 & 7 & 28 & 0.29 & 8.5 & 1 \\
\hline 144 & 144 & FREJUS & 6.665 & 45.1386 & 176473 & 10.09 & 23.9 & 254 & 85 & 124 & 3 & 34 & 4 & 26 & 0.21 & 14.9 & 2 \\
\hline 145 & 145 & GALIB & 6.4096 & 45.065 & 195495 & 6.86 & 2.9 & 281 & 12 & 30 & 57 & 184 & 30 & 35 & 0.13 & 14.1 & 2 \\
\hline 146 & 146 & GIAGLI & 7.0171 & 45.143 & 149237 & 11.86 & 10.9 & 7 & 79 & 271 & 1 & 181 & 10 & 17 & 0.56 & 15.5 & 3 \\
\hline 147 & 147 & GENEP & 6.9191 & 45.4192 & 164884 & 21.91 & 64.1 & 225 & 81 & 355 & 6 & 86 & 7 & 24 & 0.47 & 9.1 & 1 \\
\hline 148 & 148 & LANSLEV & 6.9287 & 45.2998 & 160007 & 17.55 & 8.6 & 155 & 82 & 278 & 4 & 9 & 7 & 19 & 0.26 & 11 & 3 \\
\hline 149 & & LLBOURG & 6.8697 & 45.2852 & 164031 & 16.56 & 48.4 & 156 & 54 & 335 & 36 & 65 & 1 & 30 & \# & \# & 3 \\
\hline 150 & 149 & LLBOURG2 & 6.8671 & 45.2829 & 164161 & 16.45 & 86.5 & 301 & 85 & 193 & 2 & 103 & 5 & 22 & 0.38 & 10 & 2 \\
\hline 151 & 150 & LORES & 6.9429 & 45.4013 & 162442 & 21.49 & 74.5 & 335 & 76 & 188 & 12 & 96 & 8 & 17 & 0.43 & 8.5 & 1 \\
\hline 152 & 151 & MAD & 6.368 & 45.4403 & 206547 & 18.38 & 20.6 & 127 & 27 & 320 & 62 & 219 & 5 & 26 & 0.46 & 13.3 & 2 \\
\hline 153 & 152 & MASSE & 6.6984 & 45.2596 & 176388 & 14.54 & 67.5 & 342 & 38 & 187 & 49 & 82 & 12 & 20 & \# & \# & 3 \\
\hline 154 & 153 & MASSE2 & 6.6974 & 45.2598 & 176472 & 14.54 & 5.5 & 127 & 75 & 277 & 13 & 9 & 7 & 19 & 0.56 & 7.9 & 2 \\
\hline 155 & 154 & MENU & 6.5822 & 45.3168 & 186741 & 15.82 & 22.8 & 290 & 82 & 83 & 7 & 173 & 4 & 17 & 0.46 & 5.4 & 2 \\
\hline 156 & 155 & MICHE & 6.4865 & 45.2184 & 191768 & 12.10 & 17.1 & 278 & 29 & 54 & 52 & 175 & 22 & 27 & 0.57 & 17.8 & 3 \\
\hline 157 & 156 & MODAN2 & 6.6658 & 45.198 & 182780 & 12.28 & 21.7 & 36 & 58 & 170 & 23 & 269 & 21 & 17 & 0.98 & 8.4 & 3 \\
\hline 158 & 157 & MODAN & 6.6004 & 45.2091 & 177506 & 12.20 & 76.8 & 155 & 68 & 300 & 18 & 34 & 12 & 24 & 0.53 & 12.3 & 3 \\
\hline 159 & 158 & NEIGE(1) & 7.0328 & 45.4064 & 156054 & 22.57 & 11.4 & 174 & 82 & 303 & 5 & 34 & 6 & 39 & 0.13 & 12.7 & 1 \\
\hline 160 & 159 & NEIGE(2) & 7.0328 & 45.4064 & 156054 & 22.57 & 7.6 & 285 & 9 & 92 & 81 & 195 & 2 & 14 & 0.3 & 14.4 & 2 \\
\hline 161 & 160 & OREL(1) & 6.5565 & 45.2223 & 186443 & 12.53 & 32.5 & 102 & 78 & 316 & 10 & 225 & 6 & 12 & 0.15 & 8.8 & 3 \\
\hline 162 & 161 & OREL(2) & 6.5565 & 45.2223 & 186443 & 12.53 & 57.5 & 165 & 9 & 273 & 63 & 70 & 25 & 9 & 0.06 & 5.9 & 3 \\
\hline 163 & 162 & OREL(3) & 6.5565 & 45.2223 & 186443 & 12.53 & 2.5 & 105 & 5 & 295 & 85 & 195 & 1 & 12 & 0.47 & 18.9 & 3 \\
\hline 164 & 163 & ORGIE & 6.65 & 45.2135 & 179048 & 12.67 & 0.7 & 305 & 72 & 100 & 17 & 192 & 7 & 10 & 0.36 & 7.9 & 3 \\
\hline 165 & 164 & OULX & 6.8577 & 45.0425 & 162728 & 7.02 & 22.0 & 43 & 76 & 300 & 3 & 209 & 14 & 17 & .67 & 13.5 & 3 \\
\hline 166 & 165 & PARTIE(1) & 6.6722 & 45.2584 & 178363 & 14.35 & 7.7 & 108 & 36 & 300 & 54 & 202 & 6 & 19 & 0.46 & 8.9 & 2 \\
\hline 167 & 166 & PART & 6.6722 & 45.2584 & 178363 & 14.35 & 56.3 & 316 & 85 & 48 & 0 & 138 & 3 & 10 & 0.44 & 10.8 & 3 \\
\hline 168 & 167 & PRIOUX & 6.6941 & 45.348 & 179163 & 17.58 & 56.4 & 355 & 61 & 140 & 24 & 236 & 15 & 11 & 0.13 & 5.8 & 3 \\
\hline 169 & 168 & PUIT & 6.5093 & 45.4674 & 196908 & 20.14 & 12.1 & 283 & 68 & 97 & 22 & 188 & 2 & 34 & \# & \# & 3 \\
\hline 170 & 169 & RIBON(2) & 6.9941 & 45.3104 & 155426 & 18.49 & 25. & 56 & 70 & 182 & 12 & 275 & 16 & 31 & 0.64 & 8.9 & 1 \\
\hline 171 & 170 & RIBON(1) & 6.9941 & 45.3104 & 155426 & 18.49 & 76.5 & 256 & 87 & 105 & 3 & 15 & 2 & 21 & 0.49 & 11 & 2 \\
\hline 172 & 171 & RIBON(3) & 6.9941 & 45.3104 & 155426 & 18.49 & 3.5 & 104 & 1 & 12 & 77 & 195 & 13 & 20 & 0.16 & 7.2 & 2 \\
\hline 173 & 172 & ROCHEU & 6.9818 & 45.3804 & 158791 & 21.08 & 78.1 & 79 & 73 & 211 & 12 & 303 & 12 & 15 & 0.48 & 7.6 & 2 \\
\hline 174 & 173 & ROMOL & 6.7638 & 45.1324 & 168689 & 10 & 64.2 & 52 & 71 & 214 & 18 & 306 & 5 & 22 & 0.27 & 14.4 & 3 \\
\hline 175 & 174 & SALIN & 6.528 & 45.4678 & 195531 & 20.29 & 49. & 3 & 64 & 155 & 23 & 250 & 11 & 15 & 0.93 & 8.6 & 3 \\
\hline 176 & 175 & SARRA & 6.6038 & 45.158 & 181559 & 10.54 & 22.5 & 131 & 80 & 303 & 9 & 33 & 1 & 28 & 0.26 & 21.6 & 2 \\
\hline 177 & 176 & TELEG & 6.4442 & 45.1977 & 194645 & 11.26 & 49.7 & 168 & 72 & 329 & 18 & 61 & 6 & 14 & 0.57 & 10.2 & 2 \\
\hline 178 & 177 & TERMI & 68373 & 45.2859 & 166502 & 16.36 & 33.6 & 300 & 67 & 143 & 21 & 50 & 8 & 20 & 0.01 & 9.1 & 2 \\
\hline 179 & 178 & TERMI2 & 6 & 45.3164 & 167144 & 17.50 & 43 & 21 & 70 & 247 & 14 & 154 & 14 & 24 & 0.02 & 4.8 & 2 \\
\hline 180 & 179 & VALLO & 6.4273 & 45.1594 & 195311 & 9.96 & 83.0 & 298 & 86 & 197 & 1 & 107 & 4 & 25 & 0.14 & 11.6 & 1 \\
\hline 181 & 180 & VALMO & 6.4333 & 45.4306 & 201344 & 18.50 & 4.5 & 300 & 51 & 94 & 36 & 194 & 13 & 19 & 0.11 & 2.1 & 2 \\
\hline 182 & 181 & VALTHO1 & 6.5781 & 45.2825 & 186157 & 14.66 & 9.3 & 233 & 80 & 115 & 5 & 24 & 9 & 22 & 0.59 & 18.7 & 3 \\
\hline 183 & 182 & VALTHO2 & 65660 & 45.308 & 187669 & 15.44 & 81.6 & 52 & 82 & 187 & 6 & 277 & 6 & 20 & \# & \# & 3 \\
\hline 184 & 183 & VILLARO & 7.0023 & 45.3313 & 155510 & 19.38 & 49.6 & 146 & 87 & 339 & 3 & 249 & 1 & 21 & 0.05 & 6.9 & 3 \\
\hline 185 & $\mathrm{~S} 1$ & Innri Alpa & 8.107 & 46.2143 & 159306 & 68.31 & 32.3 & 312 & 69 & 125 & 21 & 216 & 2 & 13 & 0.273 & 5.8 & 2 \\
\hline 186 & $\mathrm{~S} 2$ & Schwarzi Balma & 8.1074 & 46.2174 & 159619 & 68.37 & 9.6 & 124 & 72 & 351 & 12 & 258 & 13 & 13 & 0.296 & 10.3 & 2 \\
\hline 187 & $\mathrm{~S} 3$ & Innri Biela & 8.1041 & 46.2105 & 159003 & 68.17 & 30.2 & 300 & 80 & 129 & 10 & 38 & 2 & 10 & 0.591 & 6.9 & 3 \\
\hline 188 & S4 & Sirvultence & 7.9962 & 46.2151 & 162835 & 65.54 & 63.5 & 348 & 74 & 222 & 10 & 129 & 13 & 9 & 0.159 & 5.8 & 3 \\
\hline 189 & S5 & Obers Fulm & 7.9689 & 46.2085 & 163081 & 64.76 & 8. & 357 & 63 & 159 & 26 & 253 & 7 & 10 & 0.589 & 7.9 & 2 \\
\hline 190 & S6 & Alte Kaserne & 8.0967 & 46.1894 & 157060 & 67.66 & 11.7 & 149 & 82 & 326 & 8 & 56 & 1 & 17 & 0.401 & 5.8 & 2 \\
\hline 191 & S7 & Hopschusee & 8.0242 & 46.2543 & 165906 & 66.85 & 14.9 & 110 & 81 & 322 & 7 & 232 & 5 & 10 & 0.629 & 6.4 & 3 \\
\hline 192 & S8 & Hopschusee & 8.0222 & 46.2546 & 165990 & 66.81 & 3.8 & 128 & 70 & 336 & 17 & 243 & 9 & 13 & 0.9 & 6.2 & 2 \\
\hline 193 & S9 & Staldhorn & 8.0213 & 46.2582 & 166385 & 66.84 & 6. & 135 & 63 & 334 & 26 & 240 & 8 & 11 & 0.459 & 12.0 & 3 \\
\hline 194 & $\mathrm{~S} 10$ & Hopsche & 8.0206 & 46.253 & 165882 & 66.75 & 18.7 & 39 & 87 & 138 & 1 & 228 & 3 & 15 & 0.767 & 7.2 & 2 \\
\hline 195 & $\mathrm{~S} 11$ & Engiloch & 8.0203 & 46.2256 & 163110 & 66.30 & 8.3 & 90 & 80 & 329 & 5 & 238 & 9 & 46 & 0.508 & 11.5 & 1 \\
\hline 196 & $\mathrm{~S} 12$ & Rossbodestafel & 8.0249 & 46.1956 & 159939 & 65.93 & 21.9 & 10 & 80 & 134 & 6 & 224 & 8 & 21 & 0.439 & 11.8 & 1 \\
\hline 197 & S13 & Gali Egga & 8.0112 & 46.1919 & 160006 & 65.52 & 22.5 & 240 & 85 & 133 & 1 & 43 & 4 & 18 & 0.29 & 18.2 & 2 \\
\hline 198 & S14 & Egga & 8.0366 & 46.2034 & 160352 & 66.35 & 74.7 & 303 & 73 & 50 & 5 & 141 & 17 & 29 & 0.404 & 9.7 & 1 \\
\hline 199 & S15 & Glatthorn & 8.0714 & 46.212 & 160142 & 67.36 & 11.4 & 174 & 75 & 325 & 13 & 56 & 7 & 21 & 0.625 & 8.5 & 2 \\
\hline 200 & $\mathrm{~S} 16$ & Bodme & 8.052 & 46.1923 & 158738 & 66.56 & 27.6 & 219 & 83 & 309 & 0 & 39 & 7 & 16 & 0.346 & 6.0 & 2 \\
\hline 201 & S17 & Walderuberg & 8.0584 & 46.213 & 160638 & 67.05 & 1.1 & 134 & 82 & 337 & 7 & 246 & 3 & 17 & 0.52 & 8.4 & 2 \\
\hline
\end{tabular}


Table 1. (continued)

\begin{tabular}{|c|c|c|c|c|c|c|c|c|c|c|c|c|c|c|c|c|c|}
\hline \multicolumn{3}{|c|}{ Name } & \multicolumn{5}{|c|}{ Location } & \multicolumn{2}{|c|}{$\sigma_{1}$} & \multicolumn{2}{|c|}{$\sigma_{2}$} & \multicolumn{2}{|c|}{$\sigma_{3}$} & \multicolumn{4}{|c|}{ Parameters } \\
\hline $\mathrm{N}$ & $\mathrm{n}$ & Site & Lon & Lat & $\rho$ & $\tau$ & $\gamma$ & $\mathrm{az}$ & Dip & az & Dip & $\mathrm{az}$ & Dip & Data & $\Phi$ & $\alpha$ & Conf \\
\hline 202 & $\mathrm{~S} 18$ & Homatta & 8.0526 & 46.2171 & 161232 & 66.97 & 10.0 & 128 & 67 & 352 & 17 & 257 & 15 & 13 & 0.192 & 5.7 & 2 \\
\hline 203 & S19 & Hübschhorn W & 8.0374 & 46.2437 & 164414 & 67.01 & 15.0 & 101 & 74 & 325 & 12 & 232 & 11 & 17 & 0.686 & 9.7 & 2 \\
\hline 204 & S20 & Biel & 8.0703 & 46.1724 & 156146 & 66.70 & 35.7 & 0 & 69 & 117 & 10 & 211 & 18 & 42 & 0.16 & 10.8 & 1 \\
\hline 205 & S21 & Furggu & 8.0904 & 46.1766 & 155946 & 67.29 & 11.3 & 161 & 75 & 324 & 15 & 56 & 4 & 9 & 0.655 & 13.6 & 3 \\
\hline 206 & S22 & Guggilihorn & 8.0871 & 46.1696 & 155339 & 67.09 & 58.9 & 356 & 56 & 222 & 18 & 126 & 17 & 14 & 0.048 & 8.8 & 2 \\
\hline 207 & S23 & Bodmerhorn & 8.0413 & 46.1908 & 158924 & 66.26 & 37.3 & 70 & 72 & 302 & 12 & 209 & 14 & 17 & 0.323 & 6.9 & 2 \\
\hline 208 & S24 & Ochseläger & 7.9523 & 46.2302 & 165808 & 64.73 & 50.7 & 66 & 77 & 285 & 10 & 194 & 8 & 31 & 0.244 & 10.0 & 1 \\
\hline 209 & S25 & Lawigrabe & 8.0485 & 46.1951 & 159125 & 66.51 & 32.5 & 54 & 73 & 186 & 12 & 279 & 14 & 43 & 0.113 & 10.3 & 1 \\
\hline 210 & S26 & Bodmerhorn & 8.0397 & 46.1881 & 158705 & 66.17 & 9.2 & 103 & 73 & 329 & 12 & 237 & 14 & 31 & 0.218 & 9.0 & 1 \\
\hline 211 & S27 & Bodmer & 8.0312 & 46.1839 & 158551 & 65.89 & 17.9 & 85 & 75 & 320 & 9 & 228 & 14 & 27 & 0.283 & 9.4 & 1 \\
\hline 212 & S28 & Blauseewji & 8.0445 & 46.1847 & 158208 & 66.24 & 15.8 & 16 & 81 & 171 & 8 & 262 & 4 & 12 & 0.187 & 14.5 & 2 \\
\hline 213 & S29 & Alte Kaserne & 8.0902 & 46.1872 & 157031 & 67.45 & 22.5 & 151 & 83 & 315 & 7 & 45 & 2 & 26 & 0.513 & 9.8 & 1 \\
\hline 214 & S30 & Chaltwasser & 8.0559 & 46.249 & 164387 & 67.55 & 5.5 & 81 & 76 & 333 & 5 & 242 & 14 & 47 & 0.343 & 9.4 & 1 \\
\hline 215 & S31 & Simplon - Kulm & 8.0345 & 46.252 & 165350 & 67.07 & 13.1 & 106 & 77 & 325 & 10 & 234 & 8 & 12 & 0.519 & 6.2 & 3 \\
\hline 216 & S32 & Stockji & 7.9694 & 46.2528 & 167506 & 65.51 & 47.5 & 66 & 86 & 288 & 3 & 198 & 3 & 15 & 0.365 & 5.1 & 2 \\
\hline 217 & S33 & Antonius & 8.062 & 46.1824 & 157416 & 66.65 & 10.4 & 93 & 77 & 348 & 4 & 257 & 12 & 30 & 0.337 & 14.0 & 1 \\
\hline 218 & S34 & Färicha & 8.0515 & 46.1721 & 156707 & 66.21 & 31.2 & 308 & 79 & 125 & 11 & 215 & 1 & 27 & 0.437 & 7.9 & 2 \\
\hline 219 & S35 & Laggin Biwak & 8.0452 & 46.1654 & 156228 & 65.93 & 46.1 & 72 & 74 & 200 & 10 & 292 & 12 & 21 & 0.136 & 9.9 & 2 \\
\hline 220 & S36 & Hübschhorn $\mathrm{S}$ & 8.0547 & 46.2256 & 162039 & 67.16 & 5.2 & 67 & 73 & 332 & 2 & 242 & 17 & 34 & 0.192 & 8.6 & 1 \\
\hline 221 & S37 & Breithorn & 8.0781 & 46.2345 & 162233 & 67.88 & 4.9 & 83 & 73 & 335 & 5 & 243 & 16 & 27 & 0.325 & 5.7 & 1 \\
\hline 222 & S38 & Chesselhorn & 8.0818 & 46.221 & 160737 & 67.77 & 22.2 & 150 & 64 & 6 & 21 & 270 & 14 & 15 & 0.388 & 8.9 & 2 \\
\hline 223 & S39 & Casermetta & 8.1149 & 46.1962 & 157221 & 68.24 & 21.2 & 12 & 87 & 137 & 2 & 227 & 3 & 17 & 0.482 & 7.2 & 2 \\
\hline 224 & S40 & Waira & 8.1328 & 46.153 & 152248 & 68.03 & 13.0 & 257 & 71 & 350 & 1 & 81 & 19 & 34 & 0.266 & 12.7 & 2 \\
\hline 225 & S41 & Stalden & 7.8937 & 46.2785 & 172630 & 64.16 & 9.2 & 56 & 76 & 325 & 1 & 235 & 14 & 15 & 0.24 & 12.7 & 2 \\
\hline 226 & S42 & Furgghalte & 8.0214 & 46.2055 & 161052 & 66.00 & 19.0 & 166 & 76 & 316 & 12 & 47 & 7 & 24 & 0.501 & 9.9 & 1 \\
\hline 227 & S43 & Schilthorn & 8.0127 & 46.2105 & 161836 & 65.87 & 0.9 & 242 & 73 & 334 & 1 & 65 & 17 & 15 & 0.138 & 6.8 & 2 \\
\hline 228 & S44 & Wyssbode & 8.0179 & 46.2121 & 161824 & 66.02 & 50.0 & 284 & 70 & 24 & 4 & 116 & 20 & 12 & 0.361 & 9.5 & 3 \\
\hline 229 & S45 & Obre Stossboc & 8.0181 & 46.2022 & 160819 & 65.87 & 5.1 & 238 & 85 & 341 & 1 & 71 & 5 & 9 & 0.28 & 9.6 & 3 \\
\hline 230 & S46 & Holiecht & 8.0535 & 46.2067 & 160153 & 66 & 14.8 & 174 & 85 & 322 & 5 & 52 & 2 & 26 & 0.396 & 8.3 & 1 \\
\hline 231 & S47 & Gärtjini & 8.046 & 46.1487 & 154525 & 65.67 & 3.7 & 148 & 76 & 332 & 14 & 242 & 1 & 27 & 0.311 & 11.2 & 2 \\
\hline 232 & S48 & Weissmies & 8.0408 & 46.1416 & 153976 & 65.40 & 8.6 & 180 & 80 & 344 & 9 & 74 & 2 & 19 & 0. & 7.6 & 1 \\
\hline 233 & S49 & Schafnuwald & 8.1245 & 46.1629 & 153514 & 67.97 & 11.0 & 337 & 74 & 147 & 15 & 237 & 3 & 21 & 0.241 & 9.1 & 1 \\
\hline 234 & S50 & Tannuwald & 8.1082 & 46.154 & 153095 & 67.39 & 47.6 & 190 & 77 & 26 & 12 & 295 & 3 & 10 & 0.589 & 7.2 & 3 \\
\hline 235 & S51 & Sera & 8.1219 & 46.171 & 154425 & 68.02 & 2.0 & 5 & 80 & 160 & 10 & 250 & 4 & 9 & 0.529 & 8.0 & 3 \\
\hline 236 & S52 & Ramserna & 8.1292 & 46.2007 & 157259 & 68.68 & 24.7 & 30 & 76 & 134 & 4 & 224 & 14 & 19 & 0.423 & 7.5 & 2 \\
\hline 237 & S53 & Altes Hospiz & 8.0162 & 46.2337 & 16 & 66 & 16.3 & 121 & 73 & 322 & 16 & 230 & 6 & 27 & 0.166 & 10.0 & 1 \\
\hline 238 & S54 & Nidristi Alp & 7.9548 & 46.2578 & 168490 & 65.24 & 10.2 & 130 & 75 & 325 & 15 & 235 & 4 & 21 & 0.785 & 7.9 & 1 \\
\hline 239 & S55 & Gampisch & 8.0055 & 46.2333 & 164365 & 66.06 & 14.1 & 9 & 75 & 140 & 0 & 232 & 11 & 11 & 0.162 & 6.6 & 3 \\
\hline 240 & S56 & Zwischbergen & 8.0544 & 46.1174 & 151100 & 65.33 & 5.7 & 165 & 56 & 340 & 34 & 71 & 3 & 10 & 0.227 & 12.2 & 3 \\
\hline 241 & S57 & Galaberr & 8.0778 & 46.151 & 153737 & 66.53 & 46.5 & 24 & 72 & 203 & 18 & 293 & 1 & 19 & 0.253 & 7.9 & 2 \\
\hline 242 & S58 & Glis & 7. & 46 & 17 & 6 & 65.1 & 356 & 69 & 91 & 2 & 181 & 21 & 11 & 0 & 8.9 & 3 \\
\hline 243 & S59 & Mäderhitta & 7.9583 & 46.2838 & 170985 & 65.74 & 54.7 & 12 & 75 & 281 & 1 & 191 & 15 & 18 & 0.114 & 11.3 & 2 \\
\hline 244 & S60 & Mäderhitta & 7.9568 & 46.2763 & 170277 & 65.59 & 87.6 & 114 & 86 & 248 & 3 & 338 & 3 & 26 & 0.292 & 13.0 & 2 \\
\hline 245 & S61 & Griewald & 7.959 & 46.2868 & 171268 & 65.81 & 71.2 & 1 & 87 & 227 & 2 & 137 & 2 & 13 & 0.163 & 15.8 & 3 \\
\hline 246 & SD & ldhorn & 8.0227 & 46.2645 & 166983 & 6 & 12.0 & 145 & 4 & 357 & 85 & 235 & 2 & 11 & 0.737 & 9.7 & 3 \\
\hline 247 & 1 & TNO & 6.5208 & 44.7313 & 186998 & -4.29 & 82.3 & 142 & 77 & 349 & 18 & 258 & 6 & 30 & 0.6 & 4.7 & 1 \\
\hline 248 & 2 & TSSU & 6.529 & 44.7147 & 186555 & -4.88 & 89.9 & 108 & 83 & 356 & 3 & 265 & 6 & 25 & 0.4 & 4.8 & 1 \\
\hline 249 & 3 & RUBUR & 6.9732 & 44.3968 & 160292 & -18.99 & 68.0 & 2 & 3 & 260 & 76 & 93 & 13 & 12 & 0.8 & 17.8 & 2 \\
\hline 250 & 4 & BOUCH & 6.9492 & 44.6902 & 153907 & -7.28 & 25.7 & 64 & 69 & 236 & 21 & 327 & 3 & 30 & 0.2 & 7.5 & 1 \\
\hline 251 & 5 & & 6.7505 & 44.8862 & 167829 & 0.91 & 43.1 & 208 & 76 & 313 & 4 & 44 & 14 & 18 & 0.3 & 9.7 & 2 \\
\hline 252 & 6 & $\mathrm{ABE}$ & 6.759 & 44.973 & 167323 & 4.22 & 37.2 & 193 & 83 & 58 & 5 & 327 & 5 & 21 & 0.4 & 9.5 & 1 \\
\hline 253 & 7 & CHAMB & 6.8347 & 44.5413 & 166085 & -12.45 & 43.5 & 32 & 67 & 214 & 23 & 124 & 1 & 23 & 0.3 & 11.4 & 2 \\
\hline 254 & 8 & CHENO & 6.753 & 44.9253 & 167637 & 2.40 & 43.6 & 111 & 76 & 317 & 13 & 226 & 6 & 10 & 0.4 & 14 & 3 \\
\hline 255 & 9 & CHESU & 6.7527 & 44.9123 & 167646 & 1.91 & 3.1 & 261 & 73 & 97 & 17 & 5 & 4 & 10 & 0.3 & 11 & 3 \\
\hline 256 & 10 & MALAM & 6.6433 & 44.7118 & 177599 & -5.32 & 49.7 & 287 & 81 & 3 & 3 & 125 & 8 & 36 & 0.2 & 9.2 & 1 \\
\hline 257 & 11 & COLRI & 5333 & 44.9342 & 184994 & 2. & 6.6 & 234 & 71 & 89 & 16 & 356 & 1 & 19 & 0.3 & 7.2 & 2 \\
\hline 258 & 12 & PEYGU & 6.7403 & 44.8252 & 168851 & -1.38 & 85.6 & 66 & 78 & 182 & 5 & 273 & 11 & 13 & 0.2 & 14.6 & 2 \\
\hline 259 & 13 & LONGE & 6.9487 & 44.65 & 154686 & -8.92 & 30.9 & 81 & 70 & 296 & 17 & 202 & 11 & 16 & 0.2 & 12.9 & 2 \\
\hline 260 & 14 & COLSN & 6.841 & 44.4888 & 167107 & -14.44 & 61.6 & 202 & 3 & 354 & 57 & 104 & 13 & 15 & 0.6 & 8.6 & 2 \\
\hline 261 & 15 & CPEYR & 6.6245 & 44.7763 & 178355 & -2.97 & 20.0 & 84 & 82 & 247 & 7 & 337 & 3 & 30 & 0.2 & 11.7 & 1 \\
\hline 262 & 16 & CROUS & 6.6552 & 44.7238 & 176505 & -4.92 & 23.9 & 62 & 67 & 294 & 15 & 199 & 17 & 8 & 0.4 & 15 & 3 \\
\hline 263 & 17 & CROUS & 6.6552 & 44.7238 & 176505 & -4.92 & 73.1 & 15 & 16 & 157 & 7 & 282 & 11 & 10 & 0.5 & 16.5 & 3 \\
\hline 264 & 18 & CRXSA & 6.5508 & 44.8363 & 183762 & -0.75 & 57.8 & 143 & 82 & 327 & 8 & 237 & 1 & 19 & 0.4 & 10.3 & 2 \\
\hline 265 & 19 & CRXSA & 6.5508 & 44.8363 & 183762 & -0.75 & 80.8 & 169 & 32 & 353 & 58 & 260 & 2 & 20 & 0.5 & 7.3 & 2 \\
\hline 266 & 20 & FOUIL & 6.8003 & 44.5537 & 168416 & -11.77 & 74.2 & 130 & 82 & 4 & 5 & 274 & 6 & 11 & 0.4 & 10.4 & 3 \\
\hline 267 & 21 & GROOU & 6.6755 & 44.6593 & 175878 & -7.30 & 35.7 & 218 & 68 & 48 & 22 & 317 & 4 & 27 & 0.2 & 11 & 1 \\
\hline 268 & 22 & GROOU & 6.6755 & 44.6593 & 175878 & -7.30 & 44.7 & 219 & 39 & 37 & 5 & 128 & 1 & 27 & 0.8 & 11.3 & 3 \\
\hline
\end{tabular}


Table 1. (continued)

\begin{tabular}{|c|c|c|c|c|c|c|c|c|c|c|c|c|c|c|c|c|c|}
\hline \multicolumn{3}{|c|}{ Name } & \multicolumn{5}{|c|}{ Location } & \multicolumn{2}{|c|}{$\sigma_{1}$} & \multicolumn{2}{|c|}{$\sigma_{2}$} & \multicolumn{2}{|c|}{$\sigma_{3}$} & \multicolumn{4}{|c|}{ Parameters } \\
\hline $\mathrm{N}$ & $\mathrm{n}$ & Site & Lon & Lat & $\rho$ & $\tau$ & $\gamma$ & $\mathrm{az}$ & Dip & az & Dip & $\mathrm{az}$ & Dip & Data & $\Phi$ & $\alpha$ & Conf \\
\hline 269 & 23 & JANES & 6.7385 & 44.9338 & 168797 & 2.71 & 39.7 & 236 & 70 & 53 & 19 & 143 & 1 & 11 & 0.5 & 5 & 3 \\
\hline 270 & 24 & JANES & 6.7385 & 44.9338 & 168797 & 2.71 & 79.3 & 172 & 4 & 43 & 83 & 262 & 5 & 8 & 0.7 & 14.5 & 3 \\
\hline 271 & 25 & JANOU & 6.7535 & 44.9382 & 167624 & 2.89 & 22.9 & 343 & 81 & 250 & 0 & 160 & 9 & 13 & 0.4 & 9.8 & 2 \\
\hline 272 & 26 & LCORD & 6.7688 & 44.841 & 166517 & -0.82 & 12.2 & 204 & 84 & 77 & 4 & 347 & 5 & 13 & 0.4 & 10.5 & 2 \\
\hline 273 & 27 & NEUFC & 6.7725 & 44.5713 & 170132 & -10.96 & 68.0 & 281 & 70 & 150 & 13 & 57 & 15 & 20 & 0.3 & 10.6 & 2 \\
\hline 274 & 28 & NEUFC & 6.7725 & 44.5713 & 170132 & -10.96 & 61.0 & 16 & 20 & 208 & 70 & 108 & 4 & 12 & 0.6 & 11.4 & 3 \\
\hline 275 & 29 & LAUSE & 6.6652 & 44.8558 & 174642 & -0.17 & 82.2 & 55 & 84 & 172 & 3 & 262 & 5 & 17 & 0.3 & 8.8 & 2 \\
\hline 276 & 30 & LAVAL & 6.8773 & 44.935 & 157846 & 2.84 & 8.8 & 175 & 75 & 84 & 0 & 354 & 15 & 22 & 0.4 & 11.7 & 2 \\
\hline 277 & 31 & MROYN & 6.7297 & 44.6923 & 171090 & -6.31 & 52.7 & 32 & 18 & 204 & 72 & 301 & 2 & 10 & 0.8 & 9.4 & 3 \\
\hline 278 & 32 & ORECE & 6.5042 & 44.8017 & 187647 & -1.87 & 67.1 & 200 & 2 & 310 & 83 & 111 & 7 & 17 & 0.8 & 8.5 & 3 \\
\hline 279 & 33 & ORCEA & 6.5047 & 44.8017 & 187608 & -1.88 & 85.1 & 195 & 77 & 3 & 15 & 93 & 4 & 23 & 0.3 & 12 & 1 \\
\hline 280 & 34 & ORCEB & 6.5043 & 44.8017 & 187639 & -1.88 & 85.1 & 199 & 75 & 2 & 14 & 93 & 4 & 16 & 0.1 & 11.4 & 1 \\
\hline 281 & 35 & OREES & 6.5112 & 44.7963 & 187134 & -2.07 & 88.9 & 15 & 77 & 178 & 13 & 269 & 4 & 10 & 0.2 & 12.6 & 1 \\
\hline 282 & 36 & OREES & 6.5112 & 44.7963 & 187134 & -2.07 & 62.9 & 203 & 5 & 96 & 73 & 295 & 16 & 10 & 0.2 & 17.8 & 2 \\
\hline 283 & 37 & OREOU & 6.5115 & 44.7967 & 187107 & -2.06 & 74.9 & 192 & 15 & 18 & 75 & 283 & 1 & 26 & 0.5 & 13.5 & 3 \\
\hline 284 & 38 & OROUA & 6.5215 & 44.7952 & 186329 & -2.12 & 74.1 & 122 & 8 & 343 & 7 & 252 & 6 & 31 & 0.3 & 9.5 & 1 \\
\hline 285 & 39 & OROUB & 6.5213 & 44.7952 & 186345 & -2.12 & 89.9 & 144 & 75 & 0 & 12 & 268 & 8 & 32 & 0.2 & 11.2 & 1 \\
\hline 286 & 40 & PARPA & 6.6775 & 44.4968 & 179417 & -13.02 & 82.0 & 176 & 74 & 337 & 15 & 69 & 5 & 13 & 0.2 & 14 & 2 \\
\hline 287 & 41 & PTPUY & 6.6457 & 44.8325 & 176283 & -0.99 & 42.0 & 298 & 85 & 47 & 1 & 137 & 4 & 15 & 0.4 & 9 & 1 \\
\hline 288 & 42 & BEAUD & 6.689 & 44.8187 & 172941 & -1.55 & 48.4 & 142 & 80 & 41 & 2 & 310 & 1 & 26 & 0.3 & 15.5 & 1 \\
\hline 289 & 43 & HOUER & 6.7825 & 44.5877 & 168969 & -10.42 & 45.6 & 207 & 29 & 62 & 56 & 304 & 18 & 16 & 0.7 & 14.8 & 2 \\
\hline 290 & 44 & MLZOU & 6.6318 & 44.8422 & 177335 & -0.63 & 28.4 & 91 & 52 & 232 & 32 & 331 & 14 & 30 & 0.2 & 8.6 & 2 \\
\hline 291 & 45 & MLZSU & 6.6363 & 44.8327 & 177025 & -0.97 & 10.0 & 97 & 62 & 258 & 28 & 349 & 28 & 12 & 0.1 & 9 & 2 \\
\hline 292 & 46 & PSTRL & 6.7918 & 44.5793 & 168443 & -10.78 & 6.2 & 218 & 71 & 76 & 15 & 343 & 11 & 14 & 0.2 & 8.5 & 2 \\
\hline 293 & 47 & PCHAT & 6.7878 & 44.54 & 169738 & -12.20 & 17.8 & 210 & 69 & 64 & 18 & 330 & 11 & 29 & 0.1 & 14.3 & 1 \\
\hline 294 & 48 & PCHAT & 6.7878 & 44.54 & 169738 & -12.20 & 69.8 & 187 & 2 & 13 & 69 & 278 & 2 & 15 & 0.4 & 7.5 & 2 \\
\hline 295 & 49 & PRORE & 6.5642 & 44.9053 & 182531 & 1.64 & 45.4 & 154 & 70 & 315 & 19 & 47 & 6 & 20 & 0.2 & 9 & 2 \\
\hline 296 & 50 & RMOES & 6.6122 & 44.8347 & 178919 & -0.87 & 10.9 & 252 & 72 & 102 & 16 & 10 & 8 & 19 & 0.2 & 7.7 & 1 \\
\hline 297 & 51 & RMOOU & 6.6175 & 44.84 & 178474 & -0.69 & 1.7 & 245 & 75 & 93 & 14 & 1 & 6 & 26 & 0.3 & 8.7 & 1 \\
\hline 298 & 52 & ROBAR & 6.5698 & 44.808 & 182424 & -1.76 & 70.8 & 15 & 86 & 159 & 3 & 249 & 2 & 20 & 0.4 & 10 & 1 \\
\hline 299 & 53 & RBLES & 6.5543 & 44.9083 & 183313 & 1.74 & 21.7 & 101 & 72 & 247 & 15 & 340 & 9 & 45 & 0.5 & 9.3 & 1 \\
\hline 300 & 54 & RBLOU & 6.5423 & 44.9083 & 184260 & 1.75 & 40.7 & 312 & 71 & 50 & 3 & 141 & 18 & 22 & 0.4 & 9.7 & 1 \\
\hline 301 & 55 & TURGE & 6.7807 & 44.8288 & 165640 & -1.30 & 7.7 & 181 & 76 & 82 & 2 & 351 & 14 & 25 & 0.5 & 8.8 & 2 \\
\hline 302 & 56 & ESCPE & 6.677 & 44.6393 & 176119 & -8.02 & 75.0 & 16 & 10 & 118 & 60 & 277 & 27 & 25 & 0.2 & 7.6 & 1 \\
\hline 303 & 57 & ESCRI & 6.6898 & 44.6428 & 175053 & -7.95 & 62.0 & 205 & 38 & 10 & 52 & 110 & 7 & 34 & 0.9 & 6 & 1 \\
\hline 304 & 58 & ESCNO & 6.7003 & 44.635 & 174379 & -8.28 & 63.7 & 19 & 1 & 276 & 83 & 108 & 7 & 30 & 0.4 & 9 & 1 \\
\hline 305 & 59 & ESCSU & 6.7163 & 44.6295 & 173235 & -8.55 & 67.4 & 15 & 2 & 111 & 73 & 284 & 17 & 29 & 0.4 & 7.3 & 1 \\
\hline 306 & 60 & FREIS & 6.5357 & 44.7608 & 185505 & -3.32 & 62.3 & 213 & 83 & 329 & 3 & 59 & 7 & 15 & 0.3 & 15.8 & 2 \\
\hline 307 & 61 & FOURN & 6.5443 & 44.7962 & 184521 & -2.13 & 64.9 & 290 & 80 & 33 & 0 & 113 & 10 & 25 & 0.4 & 11.7 & 2 \\
\hline 308 & 62 & BARAI & 6.8263 & 44.5552 & 166367 & -11.88 & 45.1 & 256 & 83 & 33 & 5 & 123 & 5 & 15 & 0.4 & 14.4 & 2 \\
\hline 309 & 63 & BARAI & 6.8263 & 44.5552 & 166367 & -11.88 & 61.1 & 21 & 33 & 188 & 57 & 287 & 6 & 21 & 0.7 & 15.7 & 2 \\
\hline 310 & 64 & VMARY & 6.8625 & 44.5702 & 163187 & -11.55 & 50.5 & 50 & 73 & 206 & 16 & 298 & 7 & 17 & 0.4 & 10.5 & 2 \\
\hline 311 & 65 & VHOUE & 6.8242 & 44.5788 & 165942 & -10.99 & 56.0 & 24 & 40 & 203 & 50 & 293 & 1 & 17 & 0.8 & 9.8 & 2 \\
\hline 312 & 66 & VLAUG & 6.7508 & 44.5945 & 171279 & -9.99 & 10.0 & 325 & 79 & 89 & 6 & 180 & 9 & 6 & 0.4 & 10.3 & 3 \\
\hline
\end{tabular}

${ }^{\mathrm{a}}$ Number of the tensor in this database $(\mathrm{N})$, the number of the tensor in previous publications (n), and their names (site), the location and altitude (lon, lat in decimal degrees and $\mathrm{z}$ in meters), the polar coordinates $(r, \tau)$, and the angle with respect to the Alpine belt $(\gamma)$, the $\sigma_{1}, \sigma_{2}$, and $\sigma_{3}$ orientation (azimuth and plunge) and the $\Phi$ ratio, with $\Phi=\left(\sigma_{2}-\sigma_{3}\right) /\left(\sigma_{1}-\sigma_{3}\right)$. Also given are the number of faults used in the inversion (data), the average misfit angle $\alpha$, and the quality criterion ("conf").

an indicator for the lack of neoformed transcurrent faults. Figure $5 \mathrm{f}$ shows rose diagrams of azimuth and plunge of normal faults (top) and transcurrent faults (bottom) describing the geometrical relationship between fault orientations and kinematics.

\section{Synthesis of Brittle Deformations in the Inner Western Alps}

\subsection{Presentation of the Data}

[17] This synthesis is based on the new paleostress database presented in this paper (see section 2.2) as well as four other previously published data sets [Champagnac et al., 2003; Sue and Tricart, 2003; Champagnac et al., 2004; Grosjean et al., 2004]. The same tools for inversion were used in these studies: right diedra [Angelier, 1979] and direct inversion [Angelier, 1990], implemented in the tectonics FP [Sperner et al., 1993] and Stress [Villemin and Charlesworth, 1992] software. This provides a wide and homogenous database (312 paleostress tensors) for the entire arc of the inner western Alps, covering a surface greater than $10,000 \mathrm{~km}^{2}$ (average density of tensors: 3 tensors $/ 100 \mathrm{~km}^{2}$ ). The whole data set is presented in Table 1 and split into five zones: paleostress tensors numbered from N1 to N56 come from the south Valais (zone B, [Champagnac et al., 2003]). 

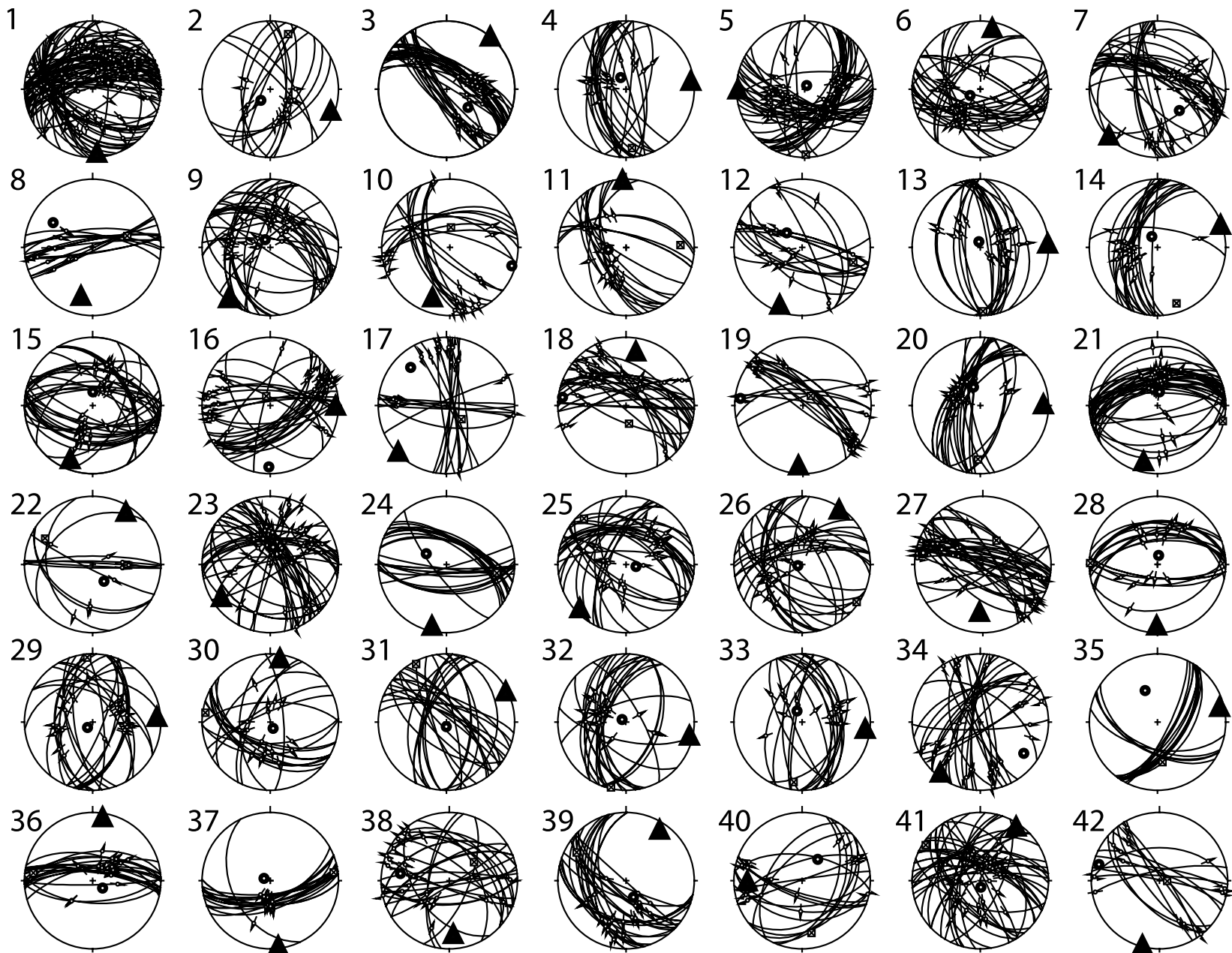

43
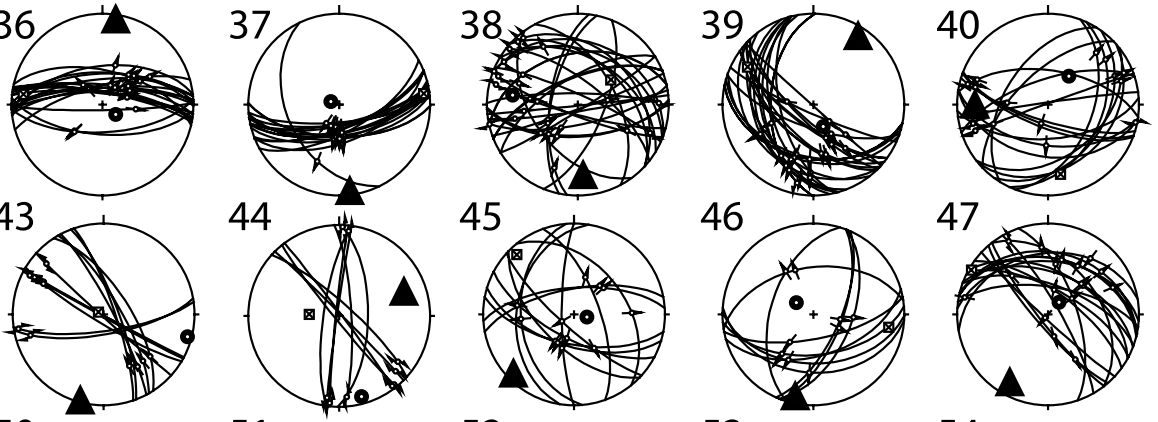

45

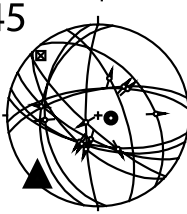

46

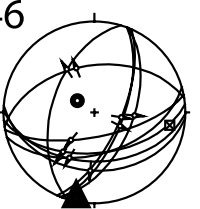

47
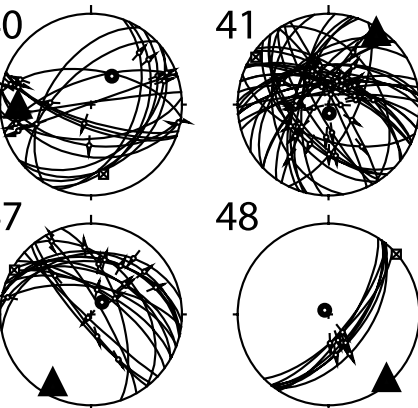

48
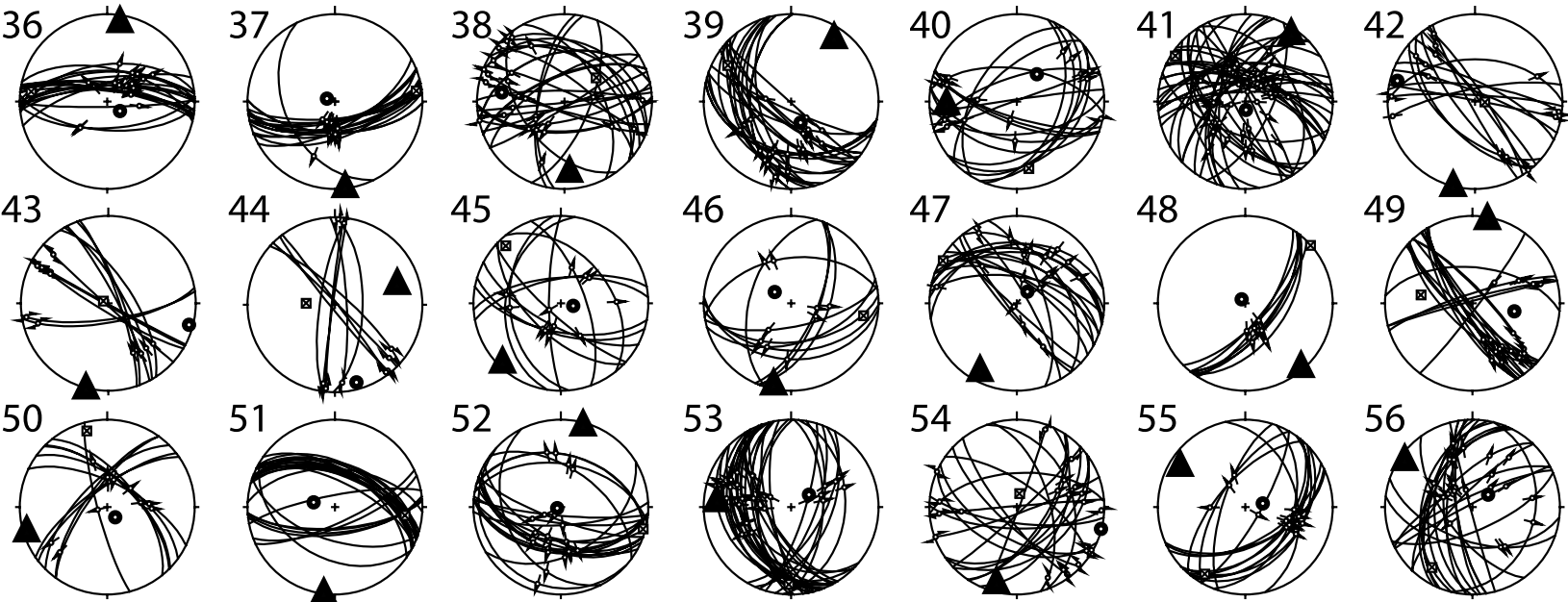

53

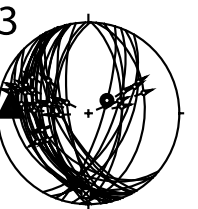

54
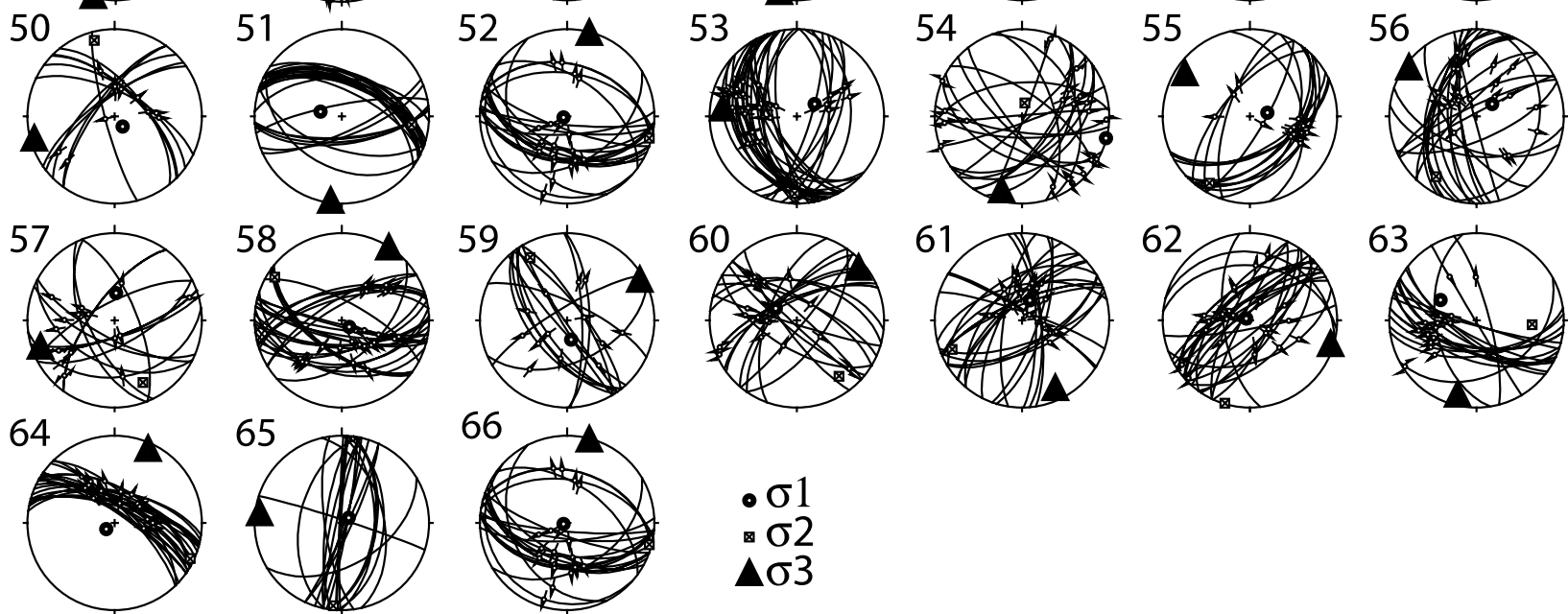

66

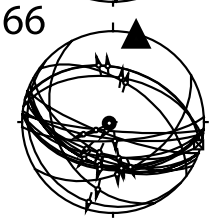

$\bullet \sigma 1$

$\triangle \sigma 2$

$\Delta \sigma 3$

Figure 3. Stereonets of the 66 unpublished paleostress tensors. As for all of the stereonets used in this paper, data are projected in a Schmidt stereogram, lower hemisphere with circles for $\sigma_{1}$ axes, squares for $\sigma_{2}$ axes, and triangles for $\sigma_{3}$ axes. Faults and striations used for the calculation are also plotted. On average, 23 fault planes and their slickenlines were used for the calculation of each tensor. 


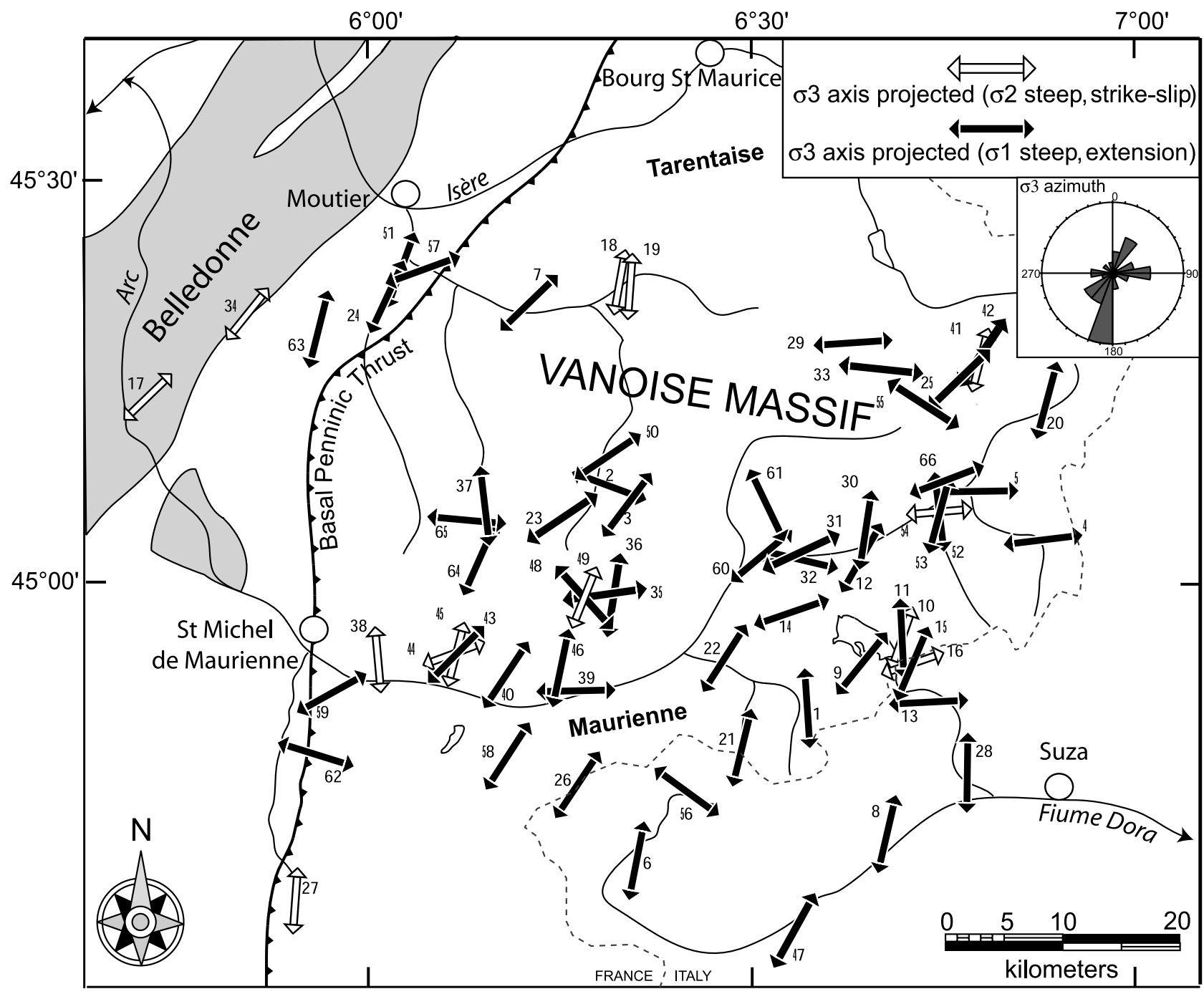

Figure 4. Paleostress direction map, with subhorizontal $\sigma_{3}$ axes projected onto a horizontal plane with white arrows for transcurrent tensors (subhorizontal $\sigma_{1}$ axis) and black arrows for extensional tensors (subvertical $\sigma_{1}$ axis). The dotted line represents the borders between Italy, Switzerland, and France; tensors are numbered from 1 to 66 beside the arrow.

Paleostress tensors numbered N57 to N118 come from the Aosta and Tarentaise valleys (zone C [Champagnac et al., 2004]). Tensors N119 to N184 are unpublished (zone D, section 2.2). Tensors numbered N187 to N246 come from the Simplon area (zone A [Grosjean et al., 2004]), and tensors N247 to N312 come from the Briançonnais area (zone E [Sue and Tricart, 2003]).

[18] In order to demonstrate the homogeneity and the large prevalence of extensional features in the inner parts of the western Alps, five examples of outcrops (one for each zone) and their associated paleostress tensors are presented in Figure 6. As in the Vanoise area, normal faulting is the most striking brittle deformation feature of the whole inner western Alps. The deformation is often penetrative (e.g., Figures $6 \mathrm{a}$ and $6 \mathrm{~d}$ ), with or without the prevalence of one of the conjugated fault families. Normal faults range from kilometer scale with a dacametric offset (e.g., Figure $6 \mathrm{~b}$ and 6c), with a clear geomorphic expression (e.g., in the form of passes in the crest line, Figure 6c), to centimeter scale, with a millimetric offset (smallest faults in Figure 6a). Figure 6e shows metric conjugated normal faulting tilted of about $30^{\circ}$ during extension. The directions of faulting observed from the landscape (or satellite) view are usually in good agreement with the general directions of the measured fault planes [Sue and Tricart, 2002; Champagnac et al., 2003].

\subsection{Synthesis and Interpretation of the Data}

\subsubsection{Directional Analyses of the Extensional Axes}

[19] Five zones are defined, corresponding to each individual study. These studies have been performed within a 

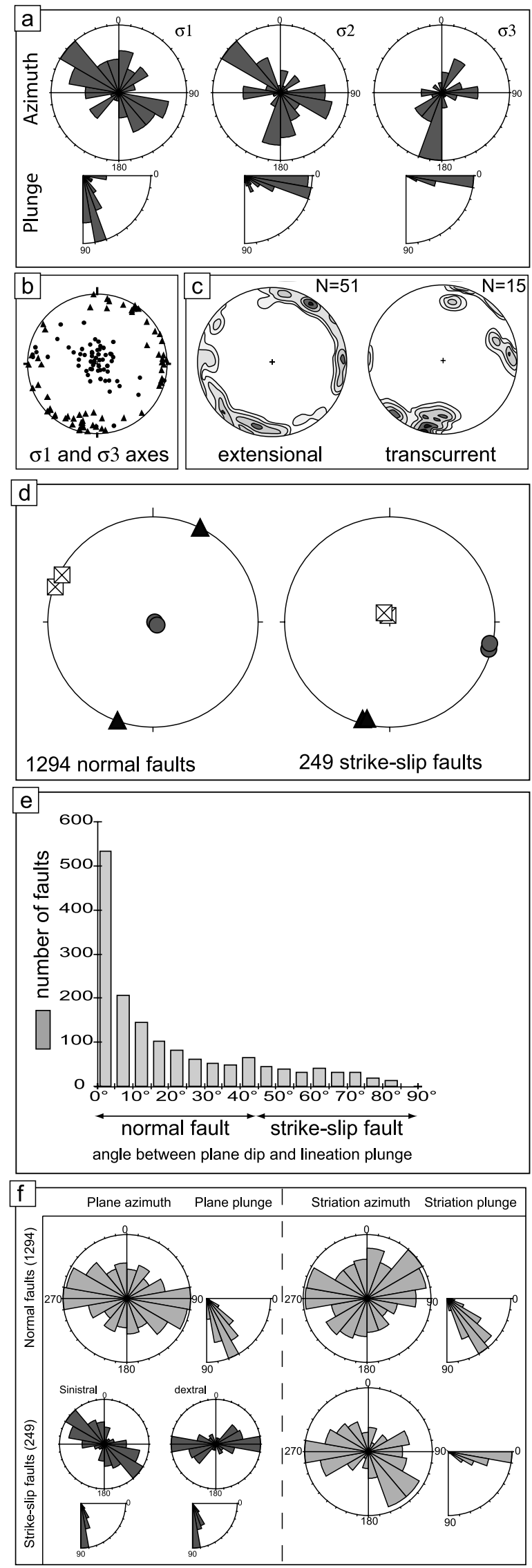

relative small area. Before carrying out a global analysis, the most remarkable features of each zone are pointed out individually (Figure 7).

\subsubsection{Zone A, Simplon Area}

[20] The data set from Grosjean et al. [2004] is located in the vicinity of the Simplon fault zone. This fault zone is a major NW-SW striking low-angle detachment, active during the Miocene [Mancktelow, 1992; Steck and Hunziker, 1994]. The total southwestward displacement of the hanging wall has been estimated to be $36 \mathrm{~km}$, with a vertical offset of about $15 \mathrm{~km}$ [e.g., Steck and Hunziker, 1994]. Brittle deformation along the Simplon fault has been documented to be active during the upper Miocene [Zwingmann and Mancktelow, 2004]. Its footwall is made of a metamorphic pile of nappes (Lepontine dome), whereas its hanging wall is composed of upper Penninic nappes, overridden by the Austroalpine low methamorphic Dent Blanche Klippe. Grosjean et al. [2004] examined the brittle deformation features associated with the Simplon fault and calculated a total of 62 paleostress tensors form some 1000 faults/striation couples. The tensors are distributed in a relatively small area, providing a very high density of data (20 tensors $/ 100 \mathrm{~km}^{2}$ ). All tensors are extensional (except N246/SD), with a stable ENE-WSW direction of extension. This orientation is identical to the pervasive stretching lineation observed within the

Figure 5. Directional analyses of the 66 tensors. (a) The $20^{\circ}$ class size rose diagram and $10^{\circ}$ class size quarter rose representing the $\sigma_{1}, \sigma_{2}$, and $\sigma_{3}$ azimuth and plunge for the 66 tensors. (b) Plot of $\sigma_{1}$ and $\sigma_{3}$ axis in a Schmidt lower hemisphere stereogram. (c) Comparison between $\sigma_{3}$ orientation for (left) extensional tensors and (right) transcurrent tensors using axes contouring mode; the major NNE-SSW directions are almost the same for both extensional and transcurrent paleostress tensors. (d) Global analysis based on the whole faults data set. Direction of extensional axis is very close for both normal faults and transcurrent faults. Calculations performed with "right dihedra" [Angelier, 1979] and dynamical-numerical methods [Sperner et al., 1993] are equivalent. (e) Histogram of the angle between fault plane dip and its striation plunge and the cumulated percentage. The $0^{\circ}$ indicates a pure dip-slip normal fault, whereas $90^{\circ}$ indicates a pure transcurrent fault. The low amount of high-angle faults indicates transcurrent movement along nonvertical fault planes (reactivated normal fault) as well as oblique striations along steep fault planes. (f) Orientation of (left) planes and (right) striations of the fault measured in the Vanoise area; (top) normal faults and (bottom) transcurrent faults are separated to determine the orientations of the faults in this area. Normal fault planes are scattered around the E-W orientation, with $50^{\circ}$ to $80^{\circ}$ dipping. Related striations are slightly extended, without clear direction; plunges vary between $40^{\circ}$ and $70^{\circ}$. Transcurrent faults are sorted by their kinematic criterion: sinistral faults are NW-SE oriented, whereas dextral faults are E-W oriented, both with subvertical dip. Striations are in agreement with plane orientations, with NW-SE and E-W main direction; plunges are subhorizontal. 

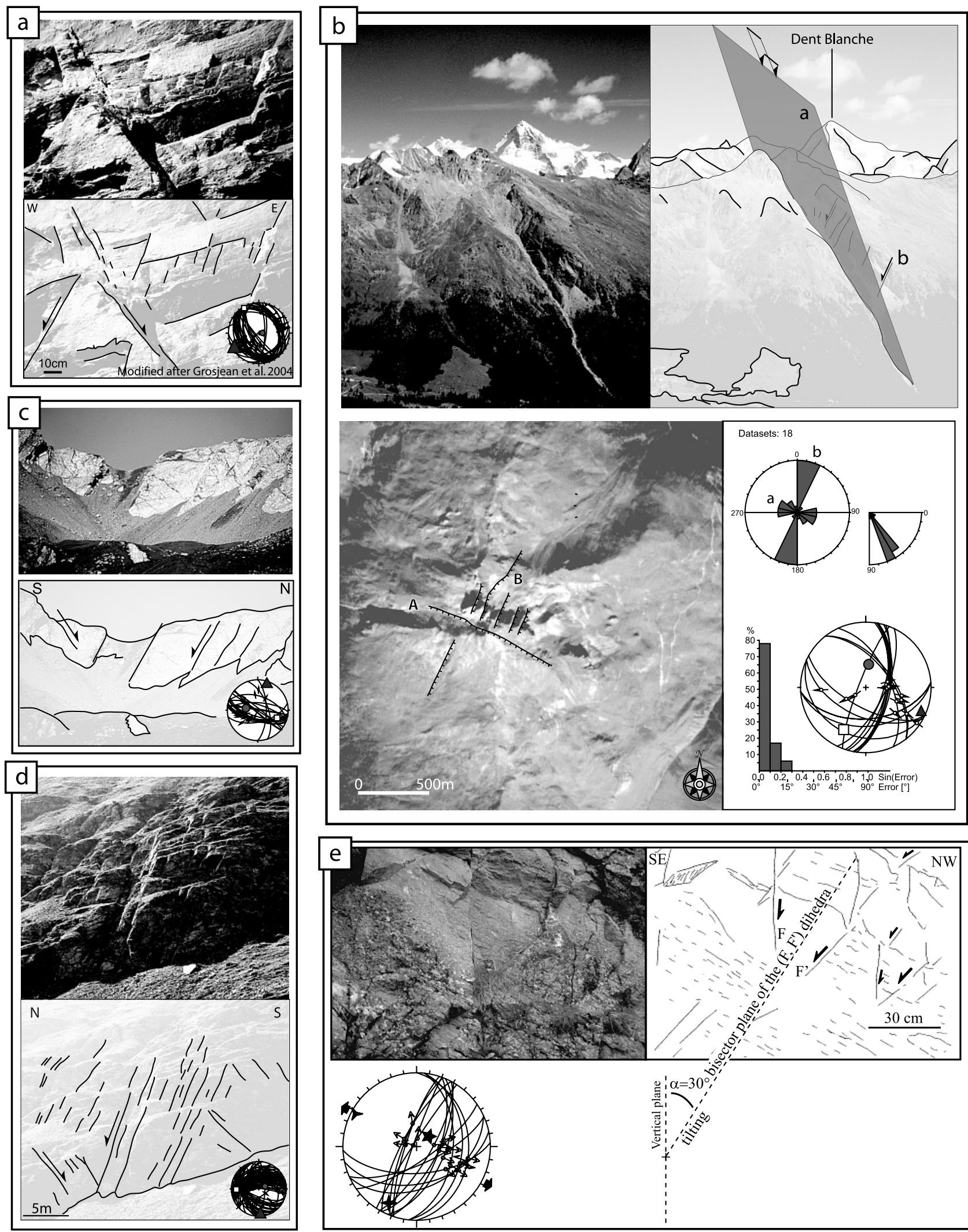

Figure 6 
Simplon mylonites by Mancktelow [1992], indicating a temporal continuum of the kinematics from ductile to brittle conditions.

\subsubsection{Zone B, Southern Valais}

[21] This zone is located immediately to the west of the Simplon area and is limited to the north by the major Rhône fault (which follows closely the present-day Basal Penninic Thrust and the Rhône valley) and to the west by the northern tip of the Mont Blanc massif. The crest line of the Combin, Dent Blanche and Monte Rosa massifs, with many summits over $4000 \mathrm{~m}$ in altitude, constitutes the southern border of this zone. The south Valais area belongs to the internal zones of the Alps and is made up of south dipping middle Penninic nappes, overridden by the main Alpine Piémont suture zone and the lower Austroalpine Dent Blanche nappe. The Briançonnais nappes consist of slices of pre-Carboniferous basement rocks, with their sedimentary cover. The Piémont suture zone is a melange of ophiolitic rocks and associated oceanic "Schistes Lustrés" calcschists. The overriding Dent Blanche nappe is the highest nappe in this part of the western Alps. It is mainly a crystalline basement unit with some isolated remnants of sedimentary cover [Steck and Hunziker, 1994; Escher and Beaumont, 1997; Steck et al., 1997]. The metamorphic history of the Penninic units is complex. Parts of the Piémont suture zone have experienced high- to ultrahigh-pressure metamorphisms, while most of the basement units have undergone only moderate pressure, middle to upper greenschist facies methamorphism [Desmons et al., 1999].

[22] Brittle deformation within this area has been examined recently [Champagnac et al., 2003]. Paleostress tensors are mainly extensional, and the mean $\sigma_{3}$ axis is oriented $\mathrm{N} 065^{\circ}$, in a stable "Simplon-like" orientation. One third of the calculated tensors are transcurrent, with a coaxial orientation of $\sigma_{3}$ axis. Relative chronologies indicate a transcurrent/transtensive stress field followed by a purely extensional stress field.

\subsubsection{Zone C, Aosta}

[23] The geological structure of this area is very similar to that of the southern Valais, with a general dip of structures to the SE. Nevertheless, slices of Valaisan units (Cretaceous flyschs) and subriançonnais units (Mesozoic marls) are squeezed between the penninic pile of nappes and the Mont Blanc External Crystalline massif. The southern part of this zone is made of internal crystalline basement of the Gran Paradiso metamorphic dome. This gneissic dome (derived from the European margin) has been metamorphosed under eclogite facies and retromorphosed under greenshist facies (see Rolland et al. [2000] for details). In the core of this zone, the large north dipping Aosta-Ranzola Oligocene normal fault [Diamond, 1990] extends from the Piccolo San Bernardo pass in the west to the Ranzola pass, more than $100 \mathrm{~km}$ to the east. Its vertical offset ranges from 0.5 to $2 \mathrm{~km}$. This major fault is described as left-lateral during Quaternary [Carraro et al., 1994].

[24] Brittle deformation analyses of this area have been published and discussed by Champagnac et al. [2004]. The stress axes orientations are quite dispersed in space, but display a main NNE-SSW orientation of $\sigma_{3}$ axis. This orientation of extension has been interpreted in terms of orogen-parallel extension. The ratio between transcurrent and extensional paleostress tensors is $1 / 4$, with varying the relative chronology.

\subsubsection{Zone D, Vanoise}

[25] This zone is presented in section 2. The ratio between transcurrent and extensional tensors is about $1 / 4$. The mean directions of $\sigma_{3}$ axes for extensional and transcurrent stress field are coaxial, N-S oriented. A minor part of extensional axes are E-W oriented, and are interpreted as an orogen perpendicular stress field, according to Champagnac et al. [2004]. N-S extension is consistent with the results of Malusa et al. [2005] in the Val di Suza, farther east.

\subsubsection{Zone E, Briançon Area}

[26] The data used in this synthesis come from Sue and Tricart [2003]. Calculated tensors and late normal faulting observations are located in the southwestern Alps, between Pelvoux and Argentera External Crystalline massif. Four major features of large-scale brittle deformation have been observed in this area: the inversion as normal fault of the former Basal Penninic Thrust, the coexistance of transverse and longitudinal normal faults, related to orogen-parallel and orogen-perpendicular extensions, respectively, and a late dextral reactivation of NNW-SSE faults. $75 \%$ of the 66 computed paleostress tensors are extensional, the remaining

Figure 6. Fieldwork example of normal faulting all around the inner Alpine belt. (a) Close-up to penetrative normal fracturation close to the Simplon pass (site N195); the inversion of the fault population measured in the vicinity of the picture site indicates a NNE-SSW direction of $\sigma_{3}$ axis. (b) Multiscale analyses of the Pic d'Artsinol area (Valais, site n3). From the NW, a large south dipping normal fault is suspected; satellite imagery (IRS image, pixel size of $6 \mathrm{~m}$ ) and field observations allow us to recognize two fault directions, a large E-W fault (south dipping, "a") and smaller NNE-SSW faults (east dipping, "b"). The $20^{\circ}$ class size rose diagram of the measured fault plane shows both " $\mathrm{a}$ " and " $\mathrm{b}$ " directions. A good quality paleostress inversion (see misfit angle histogram) demonstrates a WNW-ESE direction of extension associated with this fault pattern. (c) Large-scale E-W normal faults at the Col de Malatra (Val d'Aoste, site N75), view from the east. Normal faults cut across the limestone cliff, and create a pass about 100 meters wide between the Gran San Bernardo valley and the Val Ferret (Italy). The small-scale measured faults are also E-W oriented and the calculated $\sigma_{3}$ axis is N-S oriented. (d) Large and close spaced normal fracturation in the Val d'Ambin (site 119). Both flanks of this small valley are dissected by normal faults. The great number of fault measurements (stereoplot) allows calculation of a wellconstrained paleostress tensor with its extensional axis $\left(\sigma_{3}\right) \mathrm{N}-\mathrm{S}$ oriented. (e) Conjugated normal faults in High Ubaye (site 310). Diedra is tilted of $30^{\circ}$ toward the NW (around the $\sigma_{2}$ axis). The direction of extension $\sigma_{2}$ is NW-SE oriented. 


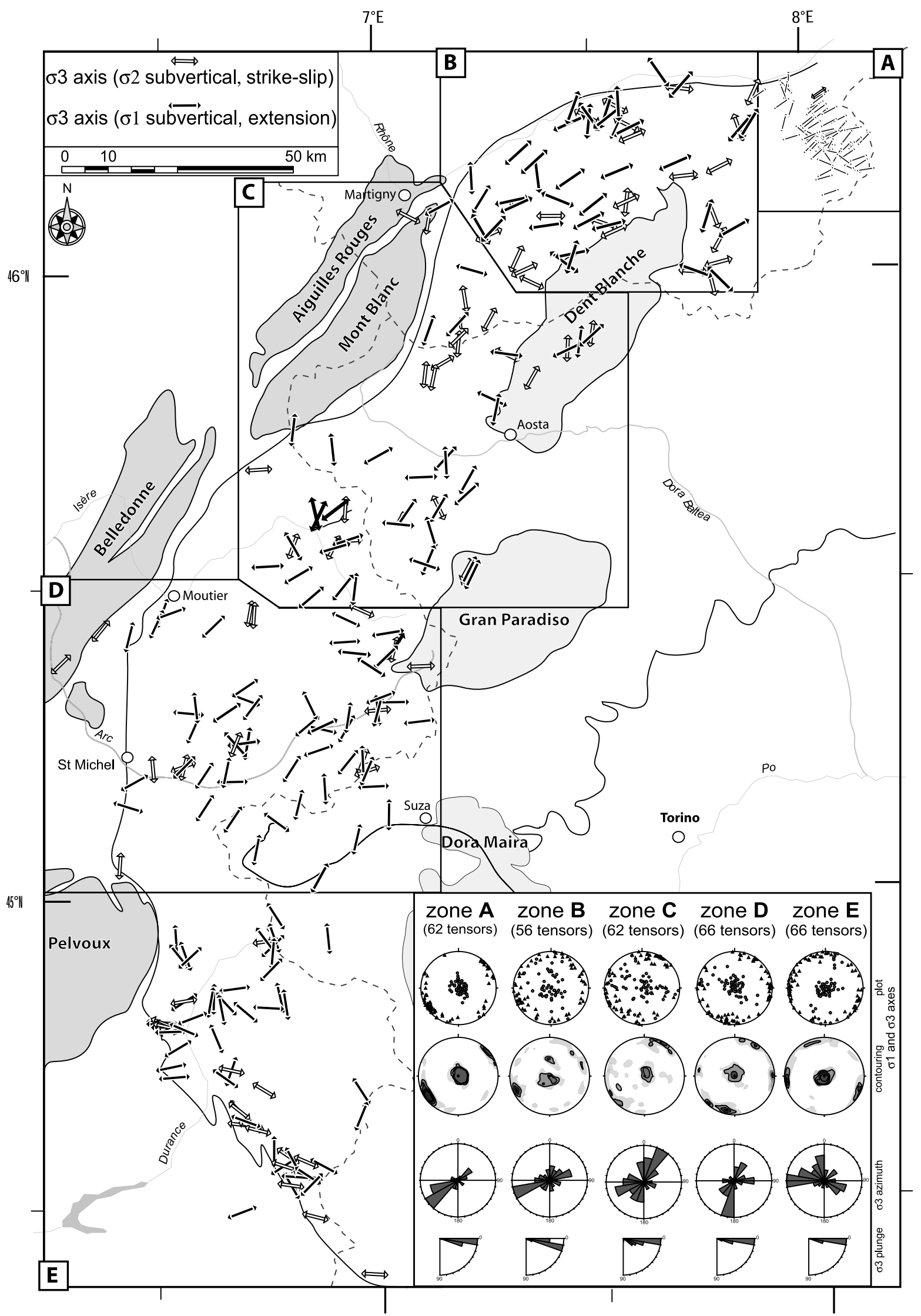

Figure 7 


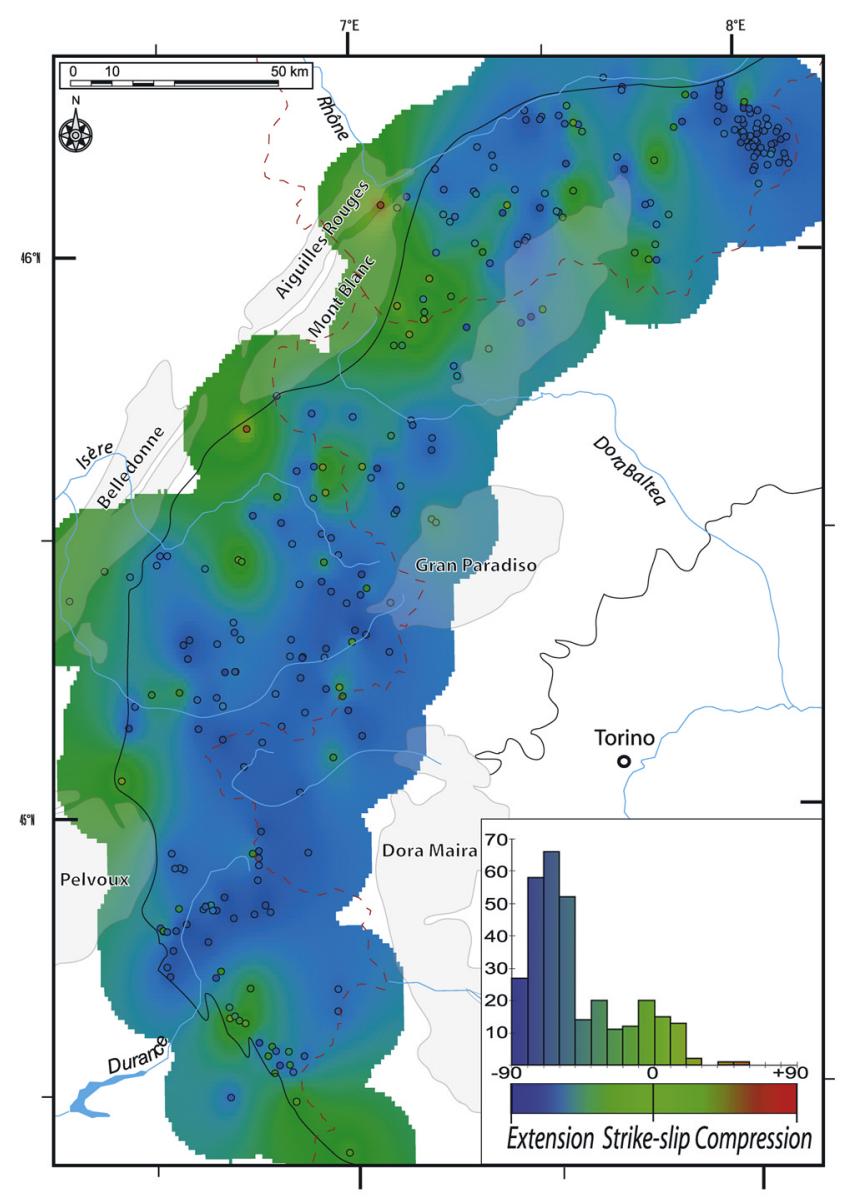

Figure 8. Contouring of the stress tensor orientation in the inner western Alps, based on the plunge of $\sigma_{1}$ and $\sigma_{3}$ axes. Color coding corresponds to the tectonic regime: compression in red, extension in blue, and strike slip in green. Small circles locate the site where paleostress inversion was performed, with their own color. The background colors come from the interpolation between data points. The blue color (extensional tectonics) largely prevails within our area of interest. The colored histogram shows the repartition of deformation regime in the belt. See text for discussion.

$25 \%$ being transcurrent. The transcurrent stress field postdates the extensional one, as demonstrated by clear crosscutting relationships. The direction of extension is E-W (perpendicular to the BPT and the general trend of Alpine structures) and N-S to NNW-SSE (parallel to the BPT and the Alpine structures). Sue and Tricart [2003] interpreted both directions in terms of what they called a "multitrend extension", based on field evidence of simultaneous faulting activity and a low $\Phi$ ratio of the calculated tensors [Sue and Tricart, 1999, 2002, 2003]. One of the more striking tectonic features of this area is the large amount of orogenperpendicular extension.

\subsubsection{Entire Data Set}

[27] Directional and statistical results of our synthesis around the Alpine belt are presented in Figure 7 . We observe a continuous change in $\sigma_{3}$ direction from $\mathrm{N} 065^{\circ}$ in the Simplon area, to N-S in the Vanoise area and to NNW$\mathrm{SSE}$ in the Briançon area. The dispersion of the $\sigma_{3}$ axes is very small in the Simplon area, probably because of the kinematic control of the major Simplon Fault Zone. The dispersion of $\sigma_{3}$ axes is greater in the Valais area, but the direction of extension is still very well constrained. The dispersion of the $\sigma_{3}$ axes increases in the Aosta/Tarentaise valleys and decreases again farther south. Another striking characteristic is the increasing amount of orogen-perpendicular $\sigma_{3}$ axes from north to south; in the Briançonnais area, the inversion of the BPT [Sue and Tricart, 1999, 2002] seems to be the origin of the E-W to NE$\mathrm{SW}$ oriented $\sigma_{3}$. The $\sigma_{1}$ axes are mainly subvertical. The dispersion of $\sigma_{1}$ axes increase from zone $\mathrm{A}$ to zone $\mathrm{C}$ and decrease from zone $\mathrm{C}$ to zone $\mathrm{E}$; the $\sigma_{1}$ axes dispersion in the Briançonnais area is comparable to the dispersion in the Simplon area.

\subsubsection{Transcurrent Versus Extensional Tectonics}

[28] The percentage of transcurrent versus extensional paleostress tensors varies from $2 \%$ (zone A) to $33 \%$ (zone B), with a mean of $25 \%$ for the whole data set. The amount of transcurrent faults is about $15 \%$ of the global data set (about 800 faults out of more than 5000). Accordingly, the transcurrent stress field appears to be a slighter signal recorded by the brittle deformation, superimposed through time onto the first-order extensional regime. In order to represent the relative importance of the different types of tectonic regimes within the internal western Alps, we calculated a variable $r$ that depends on the plunges of the $\sigma_{1}$ and $\sigma_{3}$ axes ( $r$ is plunge of $\sigma_{1}$ minus plunge of $\sigma_{3}$ ). For each tensor, the $r$ parameter is calculated and varies linearly from -90 (red, purely compressive) to 0 (green, pure strike slip) and +90 (blue, purely extensional). Intermediate values are represented by transpressive $(-90<r<0)$, and transtensive $(0<r<90)$ states of stress. We plotted every tensor with its own color code, while areas between observation points are filled with interpolation (GMT continuous curvature spline in tension [Smith and Wessel, 1990]). The map shown in Figure 8 provides an overview of the prevailing faulting styles observed all along the inner zone of the

Figure 7. Paleostress field synthesis of the inner western Alps. The map of the inner parts of the western Alps outlines some geological units (Dent Blanche, Gran Paradiso, and Dora Maira internal massifs, and Mont Blanc, Aiguilles Rouge, Belledonne and Pelvoux External Crystalline massifs). The studied area is subdivided in five parts: zone A, the Simplon pass area (with smaller arrows because of the high density of tensors), zone B, the south Valais, zone C, the Aosta and Tarentaise valleys, zone D, the Vanoise massif and the Maurienne valley and zone E, the Briançon and High Durance area (see text for details about origin of data). Arrows represent the minimum principal stress axes $\left(\sigma_{3}\right)$ projected on horizontal plane (white arrows for transcurrent tensors, black arrows for extensional tensors). The insert gives the directional analyses of paleostress orientations for the five zones separately. From top to bottom the plots of $\sigma_{3}$ (triangle) and axes (circle), the contouring of the $\sigma_{1}$ (vertical) and $\sigma_{3}$ (horizontal) axes, rose diagram and plunge of the $\sigma_{3}$ orientation. 


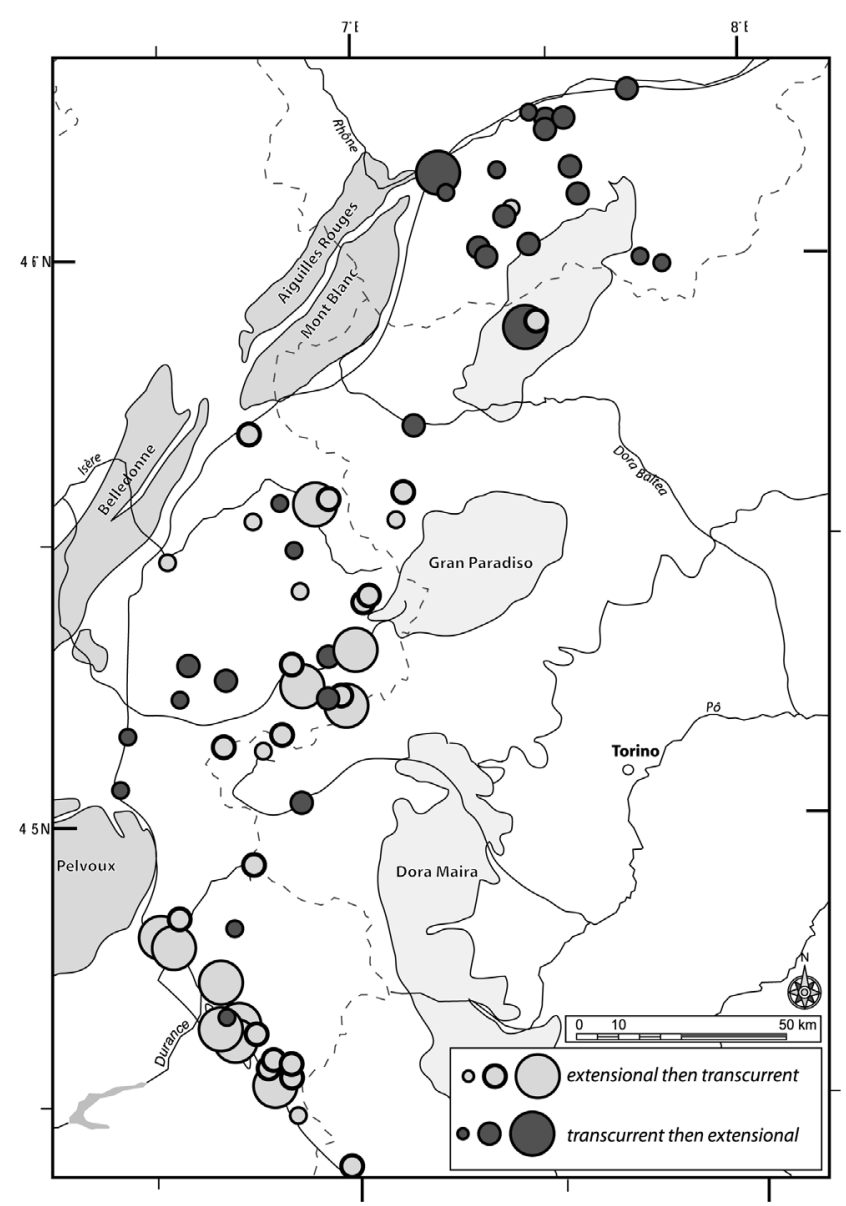

Figure 9. Chronological evidences based on field observations. The size of circles represents the reliability of the chronology (large circles for high-quality chronology evidences, small circles for lower quality). Dark grey circles symbolize locations where transcurrent deformation is older than extensional deformation. Light grey circles symbolize location where transcurrent deformation is younger than extensional deformation. The chronological orders are the opposite in the south Valais than elsewhere southward in the belt. Younger transcurrent motions are well developed in the southern branch of the arc (from the Gran Paradiso southward).

western Alps. The most striking characteristic is the predominance of an extensional state of paleostress, shown in blue. Strike slip (in green) is observed in some places, especially on the eastern side of the Mont Blanc massif, close to the Gran San Bernardo pass. This zone of transcurrent deformation separates the western Alpine belt into two parts, the Simplon and Valais areas to the north, and the Vanoise and Briançon area to the south. Southward of the Briançon area, the amount of transcurrent deformations gently increases again. Other transcurrent tensors are located on the external side of the belt, from the northern tip of the Mont Blanc massif to the central parts of the Belledonne massif. Nevertheless, site coverage in the external zones is poor, and the interpolated green colors should be regarded with some caution here.

[29] However, both transcurrent and extensional tectonic regimes are recorded by brittle deformation. In the Valais area, an older transtensive stress field, with high $\Phi$ ratio (i.e., close relative magnitudes of $\sigma_{1}$ and $\sigma_{2}$ axes, see below) is followed by a multitrend extensional stress field, with low $\Phi$ ratio (i.e., similar $\sigma_{2}$ and $\sigma_{3}$ axes) [Champagnac et al., 2003]. In contrast, the Maurienne and Briançonnais areas are characterized by younger transcurrent faults and horizontal striations superimposed on older normal faults (i.e., Figure 2c) [Sue and Tricart, 2002; Champagnac, 2005]. To better understand the spatial pattern of these chronologies, we plotted every site with the known relative chronology onto a map (Figure 9). Different circle sizes have been used to express the reliability of our observation (big circles indicate a well-established chronology, small circles indicate a low degree of confidence). This map confirms the intuitive observation of opposite chronologies between the Valais and all the surrounding areas. For most of the inner western Alps, the chronology is clear: extension is older than strike slip. The Valais area is however an exception where strike slip movements are followed by extension. The transition between the two regimes is located to the east side of the Mont Blanc massif, an area characterized by a predominance of transcurrent paleostress tensors, as highlighted in Figure 8. Despite these differences along strike, the $\sigma_{3}$ axes orientations for extensional and transcurrent stress fields show no statistical differences; moreover, an orientation analysis performed with the entire data set of normal faults and transcurrent faults within zones B, C and $\mathrm{D}$ show less than $10^{\circ}$ difference between the transcurrent $\sigma_{3}$ axis and the extensional $\sigma_{3}$ axis (e.g., Figures $5 \mathrm{c}$ and $5 \mathrm{~d}$ and Champagnac [2005]). Accordingly, we suggest a swapping between $\sigma_{1}$ and $\sigma_{2}$ axes in a single "deformation phase," with instabilities in space and time modifying the overall stress field [Champagnac et al., 2003; Sue and Tricart, 2003; Champagnac et al., 2004]. A part of such stress axes permutations could also be induced by rock heterogeneity and anisotropy, as demonstrated by Hu and Angelier [2004]; this phenomenon could be important, and needs to be considered. Nevertheless, chronological evidence (Figure 9) indicates opposite chronologies between the Valais and remaining parts of the inner western Alps, and the chronologies probably have a geological significance.

\subsection{Ellipsoid Shape Form Parameter $\Phi$}

[30] Beyond the stress orientation, paleostress inversion allows one to determine the relative magnitude of $\sigma_{1}, \sigma_{2}$ and $\sigma_{3}$ of the stress ellipsoid, the $\Phi$ ratio; $\Phi=\left[\sigma_{2}-\sigma_{3}\right] /\left[\sigma_{1}-\right.$ $\left.\sigma_{3}\right]$ ) and $0<\Phi<1$ [e.g., Angelier, 1979]. A low $\Phi$ ratio (similar $\sigma_{2}$ and $\sigma_{3}$ ) indicates a multitrend (i.e., symmetric around the vertical axis) extension or transpression (for extensional or transcurrent tensor, respectively), and high $\Phi$ ratio (similar $\sigma_{1}$ and $\sigma_{3}$ ) indicates a transtensional stress field. According to our calculations, the $\Phi$ parameter provides a powerful and sensitive tool to investigate subtle differences in paleostress data, but needs to be used with caution, because of its high sensitivity to heterogeneous 

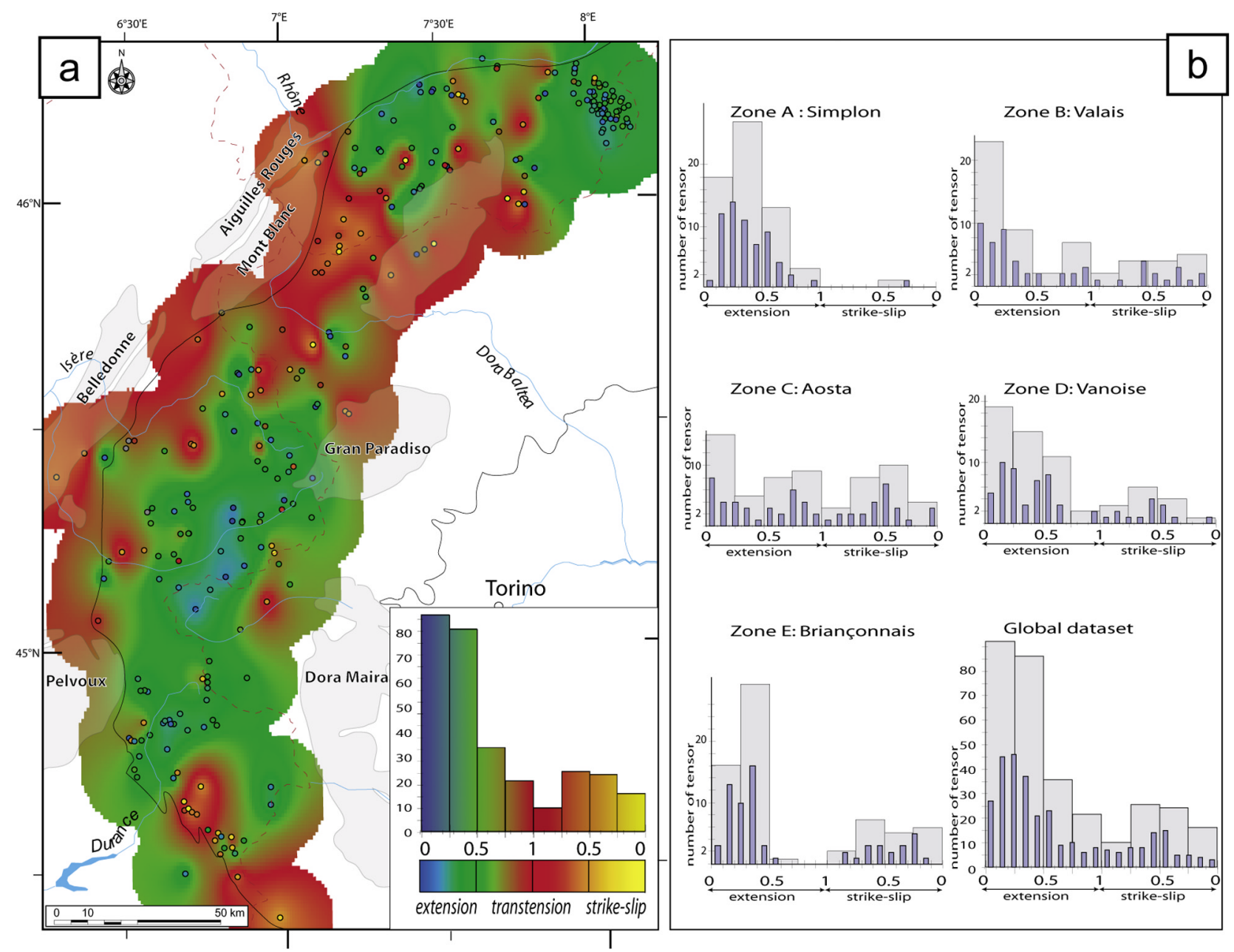

Figure 10. Map of the $\Phi$ ratio in the inner western Alps. (a) Map with interpolated $\Phi$ ratio for the entire data set. Color code is used between $\Phi=0$ (blue) and $\Phi=1$ (red), via $\Phi=0.5$ (green), for extensional tensors and between $\Phi=1$ (red) and $\Phi=0$ (yellow) via $\Phi=0.5$ (orange) for transcurrent tensors. Colored histogram gives the repartition of $\Phi$ ratio of the inner western Alpine belt. (b) Histogram presentation of the $\Phi$ ratio for each of the five zones separately, and for the entire data set, within $10^{\circ}$ and $25^{\circ}$ class size. The meaning of $\Phi$ ratio differs according to the plunge of $\sigma_{1}$, the histograms are split into two parts: from 0 to 1 for extensional tensors and then from 1 to 0 for transcurrent tensors.

data sets. We present this parameter on a map (Figure 10a) and some further statistical analyses we performed (Figures 10b). Given the fact that the significance of a low $\Phi$ ratio is different for extensional or transcurrent tensors, we used a different color coding scheme: a blue-green-red scale is used for extensional tensors $\Phi=0$ (blue) and $\Phi=1$ (red), through $\Phi=0.5$ (green), whereas a red-orange-yellow scale is used for the representation of transcurrent tensors $\Phi=1$ (red) and $\Phi=0$ (yellow) through $\Phi=0.5$ (orange).

[31] Spatial interpolation has been performed between the documented sites (same interpolator as for $r$ parameter, compare Figure 8). The map (Figure 10a) appears with large green areas, revealing pure extension. Some zones appear with blue tones, especially in the western Valais, the Simplon and the Vanoise area. A striking feature is the large reddish area in the Aosta valley, at the eastern side of the
Mont Blanc massif. A large transtensive to transcurrent region has recorded strike slip rather than the extensional tectonics prevailing in surrounding areas. This transcurrent/ transtensive area coincides with the transitions in the relative chronology described above (Figure 9). The position of this area (Val d'Aosta and surrounding) with respect to the Mont Blanc massif and/or the arc of the Alps (transition between central Alps and western Alps) could have reduced the extensional processes and thus increase the transcurrent tectonic. Such geodynamical process(es) remains open to debate. Other red/orange areas are located at the western flank of our studied area, along the External Crystalline massifs, and in the southernmost areas, close to the Basal Penninic Thrust. To better understand the spatial patterns and the relative values of the $\Phi$ ratio, we also plotted them in histograms, one for each zone and one for 

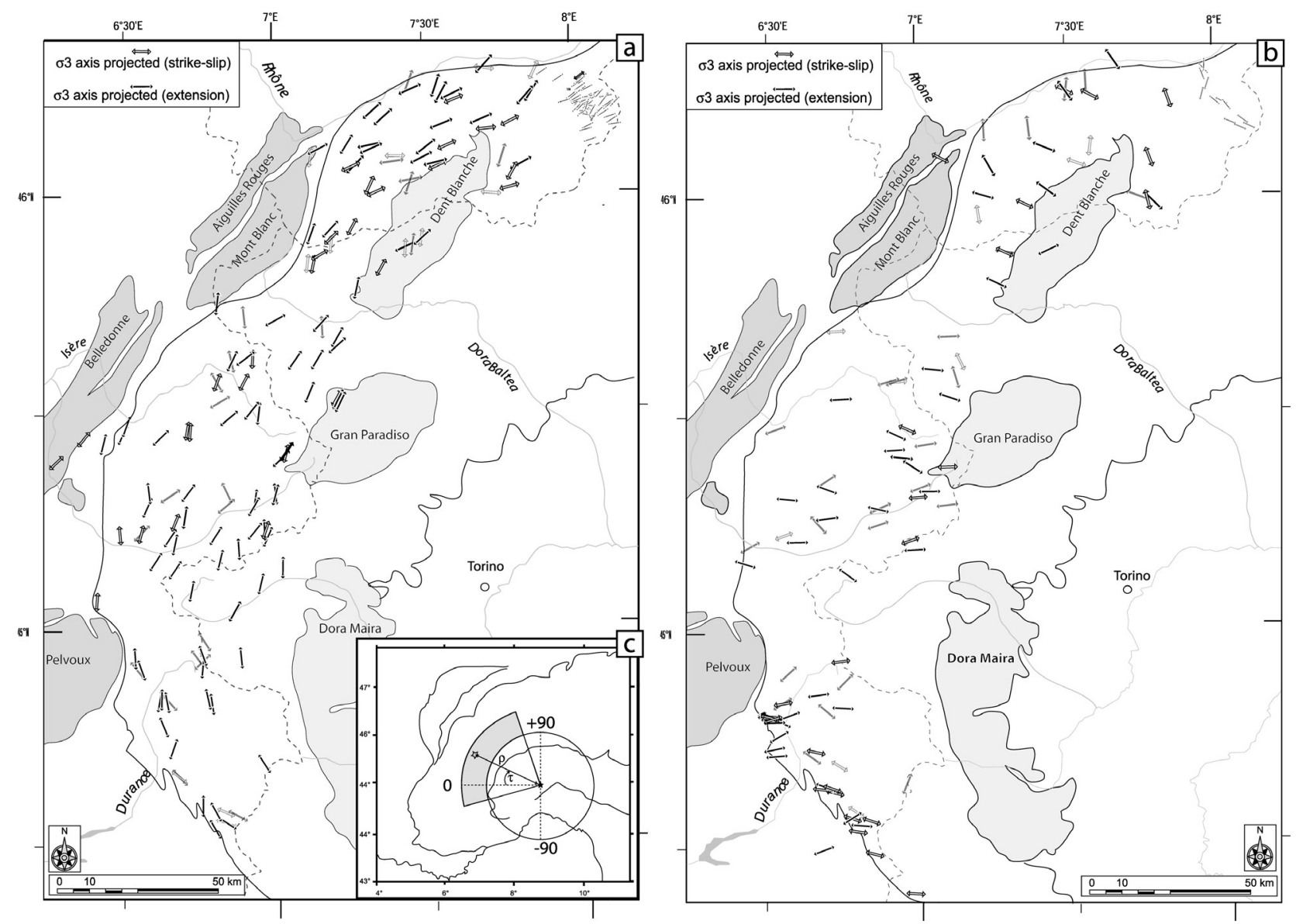

Figure 11. Spatial repartition of the extensional axes with respect to the Alpine belt. (a) Orogen-parallel extensional axes $\left(\beta<30^{\circ}\right.$, see text for details) plotted onto an Alpine map with white arrows for transcurrent and black arrows for extensional paleostress tensors. Also are plotted tensors with $30^{\circ}<\beta<$ $45^{\circ}$, in light grey. (b) Orogen-perpendicular extensional axes $\left(\beta>60^{\circ}\right)$ plotted onto Alpine map, with the same color code as Figure 12a $\left(90^{\circ}>\beta>60^{\circ}\right)$ in light grey. Outline of western Alps and circles used to convert geographical coordinates into polar coordinates are shown. The grey bend represents the area where we performed paleostress calculation $\left(-19^{\circ}<\tau<69^{\circ}\right.$ and $\left.147.8 \mathrm{~km}<r<246.5 \mathrm{~km}\right)$.

the whole data set (Figure 10b). Histograms (number of observations versus $\Phi$ ratio) were again plotted separately for extensional and transcurrent tensors. We further arranged the abscissas of the histograms $(\Phi)$ in order to show a continuum from multitrend extension to transpressive tensor, via pure extension, transtension and pure strike slip. The histogram of the global data set shows a peak for extensional low $\Phi$ ratio $(0<\Phi<0.5$, multitrend extension $)$ and a minimum for high $\Phi$ ratio $(0.75<\Phi<1$, transtension $)$ for both extensional and transcurrent tensors. A small second-order mode appears for transcurrent medium $\Phi$ ratios (close to 0.5). In the zone A (Simplon area), the tensors are exclusively extensional, and the $\Phi$ ratio is close to 0.5 (pure extension). In zone B (Valais area), most of the tensors have a low $\Phi$ ratio (less than 0.3, multitrend extension), with a second-order mode for high $\Phi$ ratios (transtension). Zone C (Aosta area) is characterized by a wide range of $\Phi$ ratios and with three small maxima (multitrend extension, transtension and pure strike lip). The Vanoise area (zone D) presents a relatively wide distribution of extensional tensors $(0<\Phi<0.75)$, with a peak of low values, and another ample maximum for transcurrent medium $\Phi$ value (pure strike lip). The southernmost area (Briançonnais area, zone E) is characterized by a bimodal histogram, with a first peak of medium to low $\Phi$ ratio within extensional tensors $(0.1<\Phi<0.4)$ and a second, more diffuse maximum for transcurrent tensors $(0.7>\Phi>0.2)$.

[32] To sum up, the $\Phi$ ratio analysis shows mostly bimodal distributions, clearly visible for zone D, E and the whole data set. For extensional tensors, $\Phi$ ratios are shifted to minor values, except in the Simplon area. The most impressive shift is observed in the Valais area, with very a low $\Phi$ ratio. This shift could be related to partly 

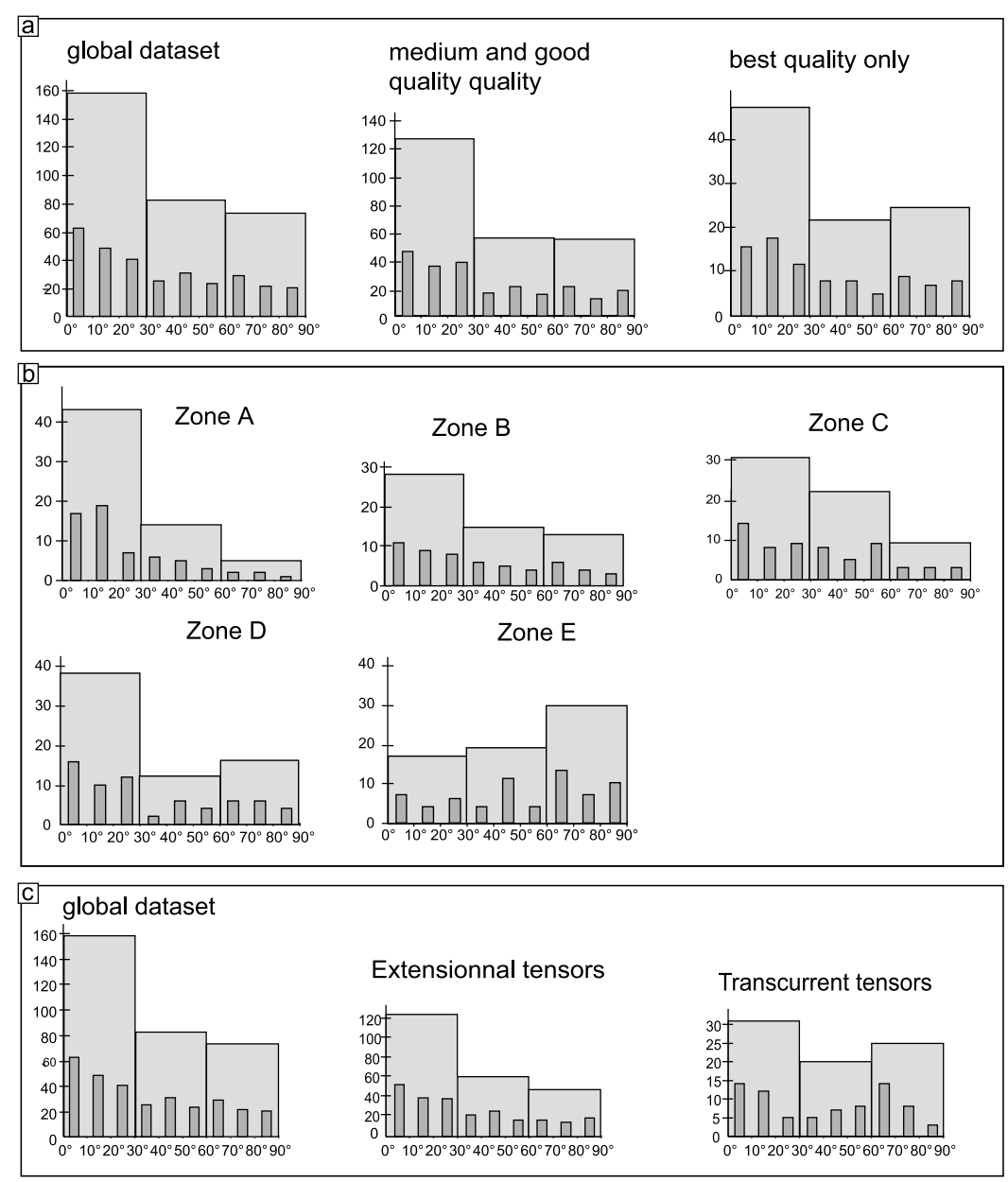

Figure 12. Histograms of the angular relationship between the Alpine trend and $\sigma_{3}$ orientations. (a) Angles are plotted within $10^{\circ}$ and $30^{\circ}$ class size for (left) the whole data set, (middle) medium and good quality criterion tensors, and (right) only best quality tensors. Despite the ever-predominant orogen-parallel tensors, best quality tensors appear to be slightly more "orogen-perpendicular" than lower quality. (b) Histograms of $\beta$ angles for each zone separately, within $10^{\circ}$ and $30^{\circ}$ class size. See text for discussion. (c) Angular relationship between extensional axes $\left(\sigma_{3}\right)$ plotted into histograms with $10^{\circ}$ and $30^{\circ}$ class size histograms for (left) global data set, (middle) extensional tensors, and (right) transcurrent tensors. See text for details.

heterogeneous data set, but probably reflects two directions of extension occurring in the same time (transition between two tectonic regime, see below).

\subsection{Stress Axes in "Polar Alpine" Coordinates}

[33] Given the arcuate shape of the western Alpine belt, the directional analyses of extensional axes, such as shown in Figure 6, as well as the choice of subsets (5 areas) is always biased with respect to the continuously changing strike direction of the bend of the NW Alps. For instance, a $\mathrm{N}-\mathrm{S}$ direction in the Valais area has the same meaning as an $\mathrm{E}-\mathrm{W}$ direction in the Vanoise area, if we consider the stress directions with respect to the strike of the Alps. In order to take this problem into account, we adopted a new coordinate system of polar coordinates. The shape of the northwestern Alpine belt can indeed be approximated with a portion of a circle, matching closely the changes in strike observed both at the internal and external side of the Alps, as well as all first-order structures (External Crystalline massifs and Basal Penninic Thrust) as seen in map view. We have chosen a centre for our circle located in the middle of the Po plain (longitude $45^{\circ} \mathrm{N}$, latitude $8.87^{\circ} \mathrm{E}$, Figure 11a). Polar coordinates have been determined for all measurement sites with $\rho$ the radius in kilometers from the center and $\tau$ the angular position in the Alpine belt, in degrees (measured positively in a clockwise sense from the west, Table 1).

[34] In order to examine the angular relationships between the arc of the NW Alps and paleostress axes orientations, we considered the three following angles: $\beta$ the $\sigma_{3}$ axis azimuth (with $0^{\circ}<\beta<180^{\circ}$ ), $\tau$ the angular position of the tensor in the Alpine belt (with $-19^{\circ}<\tau<69^{\circ}$ here) and $\gamma$ the angular position of the $\sigma_{3}$ axis with respect to the Alpine belt (with $\left(\gamma=\operatorname{abs}(\beta-\tau)\right.$ ) and (if $\gamma>90^{\circ}$, then $\gamma=$ $180-\gamma))$. 

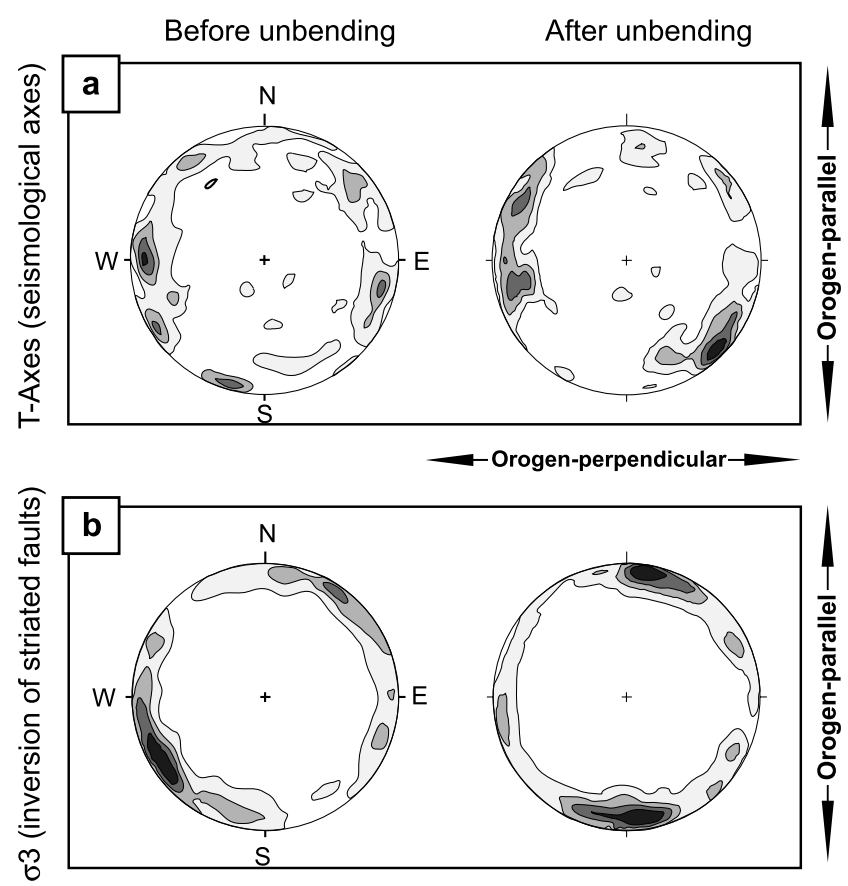

Figure 13. Contouring plot of seismotectonic $\mathrm{T}$ axes and $\sigma_{3}$ orientation calculated from fault measurements (Gauss counting method with $\mathrm{k}=100$ [Robin and Jowett, 1986], contours at 1, 2, 3, and 4). (a) T axes from Delacou et al. [2004] database within our area of interest (grey bend of the Figure 11c). (left) Stereoplot of a classical contouring of T axes, in the geographical coordinates. (right) Stereoplot of the orientation of $\mathrm{T}$ axes with respect to the Alpine structures ("Alpine polar" coordinates). E-W to NW-SE orientations indicate an orogen-perpendicular direction of extensional axes. (b) The $\sigma_{3}$ (this paper) contouring (left) in geographical coordinates and (right) with respect to the Alpine structures ("Alpine polar coordinates"). The main orogen-parallel signal ("N-S") appears clearly, whereas the orogen-perpendicular ("E-W") is less expressed.

[35] The angular results are provided in Table 1 ( $\rho$ and $\tau$ entries), and the pattern of $\sigma_{3}$ axes with respect to the Alpine belt is plotted onto maps (Figure 11). On the basis of these angles, it is now possible to compare the direction of the extensional axis (for each tensor) with respect to its position within the Alpine belt. We adopt the following terminology: "orogen-parallel extension" is characterized by $0^{\circ}<\sigma_{3}<30^{\circ}$; "orogen-perpendicular extension" is characterized by $90^{\circ}>\gamma>60^{\circ}$ and "oblique extension" is characterized by $30^{\circ}<\gamma<60^{\circ}$. As shown in Figure 11a, the NW Alps are dominated by an orogen-parallel extension, prevailing within a wide belt of the inner Alps. Additionally, a large fan of orogen-perpendicular $\sigma_{3}$ axes is apparent from Figure $11 \mathrm{~b}$. This tendency is especially significant in the southern part of the belt (from the Vanoise area southward). Histograms (Figure 12c) give details of the distribution of $\sigma_{3}$ axes orientations with respect to the Alpine structures. The distribution for all the tensors is roughly $50 \%$ for orogen-parallel extensional axes, $27 \%$ and $23 \%$ for oblique and orogen-perpendicular extensional axes, respectively. With histogram classes of $10^{\circ}$, the mode is within the $0^{\circ}-$ $10^{\circ}$ class (orogen-parallel) with more than 60 tensors $(20 \%)$. The distribution for extensional tensors is almost the same, with a mode in the $0^{\circ}-10^{\circ}$ class. In contrast, there is a bimodal distribution for transcurrent tensors; a first mode lies in the $0^{\circ}-10^{\circ}$ class and a second one in the $60^{\circ}-70^{\circ}$ class; the relative importance of orogen-perpendicular extensional axes is about $35 \%$. In order to test the robustness of our tensor directions, we established an additional series of histograms (Figure 12a) with the angular distribution of extensional axes for good tensors (quality criterion of 1 or 2 ) and for the best tensors exclusively (quality criterion of 1 ). There is no noticeable change in the histogram shape, but the relative importance of orogen-perpendicular tensors increases, whereas the number of oblique tensors gently decreases. This could lead us to consider some oblique tensors as orogen-parallel (or -perpendicular) shifted by measurement (or calculation) imprecisions or block rotations. Of course, oblique tensors could also be regarded as true local direction of extension. The angle between the Alpine structures and extensional axes distribution of the stress axes for the five Alpine zones defined above is presented in Figure 12b. Zone A (Simplon area) presents many orogen-parallel $\sigma_{3}$ axes (more than $2 / 3$ ) and very few orogen-perpendicular tensors; zone $\mathrm{B}$ and $\mathrm{C}$ have almost the same distribution as the global data set. As already seen in Figure $6 \mathrm{~b}$, zone $\mathrm{D}$ presents a bimodal distribution with a small maximum for orogen-perpendicular extensional axes. Zone $\mathrm{E}$ is the only area with more orogen-perpendicular than orogen-parallel extensional tensors; this has been interpreted by the normal reactivation of the Basal Penninic Thrust [Sue and Tricart, 1999, 2002]. From a global perspective, the relative amount of orogen-perpendicular paleostress tensors increases from north to south.

[36] In order to compare the paleostress results with the current direction of extension based on the inversion of focal plane mechanisms of earthquakes [Delacou et al., 2004], we used the same polar coordinates to analyze seismotectonic extensional axes. T axes come from Delacou et al. [2004] and have been chosen only within the area of our fault analysis $\left(-19^{\circ}<\tau<69^{\circ}\right.$ and $147.8 \mathrm{~km}<\rho<$ $246.5 \mathrm{~km}$ ). We then plotted the orientation of $\mathrm{T}$ axes (seismotectonics) and $\sigma_{3}$ axes (brittle deformation) in geographic coordinates $(\beta)$ and in Alpine angular coordinates $(\gamma)$, with N-S orientation corresponding to orogen-parallel extension and E-W orientation corresponding to orogenperpendicular (Figure 13). The seismotectonic T axes contouring (Figure 13a) shows no obvious direction of extension, because of the curved shape of the NW Alps. In polar coordinates, however, a systematic pattern appears, oriented almost E-W to WNW-ESE, that is to say, perpendicular to the Alpine structures. In contrast, the contouring of $\sigma_{3}$ axes (Figure 13b) shows a NE-SW general trend of extension, which becomes "N-S" in polar coordinates, that is to say, orogen-parallel, with a minor portion E-W oriented.

[37] To sum up, the polar representation of extensional axes in the western Alps indicates major orogen-parallel (and a minor orogen-perpendicular) extension for Neogene 
brittle deformation, whereas current seismotectonics $\mathrm{T}$ axes are clearly orogen-perpendicular oriented.

\section{Discussion}

\subsection{Overview of Present-Day Deformation}

[38] While convergence between Africa and Europe is well established on the larger scale, with a rate of 3 to $8 \mathrm{~mm} / \mathrm{yr}$ at the longitude of the western Alps [DeMets et al., 1994; Nocquet and Calais, 2004], recent geodetic surveys [Sue et al., 2000; Calais et al., 2002; Oldow et al., 2002; Serpelloni et al., 2005] failed to demonstrate any convergence across the western Alps. Rather, the present-day deformation along the Lyon-Torino baseline is extensional, with a very low rate $(<1 \mathrm{~mm} / \mathrm{yr})$ [Calais et al., 2002]. Relative movements between the northern part of the Apulian microplate and stable Europe appear to be insignificant [Oldow et al., 2002, Serpelloni et al., 2005]. The large-scale Africa-Europe convergence is consumed elsewhere, most likely south of Tyrrhenian Sea [Serpelloni et al., 2005], and within the Magrebides, as well as partly within the Dinarides, Appenines, and eastern Alps [Serpelloni et al., 2005].

[39] Seismological studies have been performed in the Alpine belt for decades [Pavoni, 1980; Béthoux et al., 1988; Deichmann and Rybach, 1989], but the extensional regime has been discovered only recently [Eva et al., 1998; Sue et al., 1999; Baroux et al., 2001; Delacou et al., 2004; Kastrup et al., 2004]. This extensional regime is well developed all along the core of the western Alps. As demonstrated by Delacou et al. [2004], this extensional deformation is associated with overthickened crust and a negative Bouguer anomaly. The directions of seismological $\mathrm{T}$ axes, as well as $\sigma_{3}$ axes (from the inversion of earthquake populations) describe a regular fan-shaped pattern, perpendicular to the Alpine belt (Figure 13). Only few compressional earthquakes have been recorded, and they are mainly located at the transition of the Alps with the foreland/lowland. The orogen-perpendicular extension is directionally consistent with the orogen-perpendicular extension we determined with our fault analysis (Figure 11b).

\subsection{Neogene Brittle Deformation}

[40] In this paper, we examined the brittle extensional tectonics in the inner western Alps. The majority $(\sim 80 \%)$ of the calculated paleostress tensors are extensional with only few transcurrent tensors. More than $85 \%$ of all measured faults are normal, $15 \%$ are transcurrent. Transcurrent tectonics is the last event in the southern parts of the inner western Alps, whereas it is preceding extension in the Valais area (see section 3.2.2). The limit between these contrasting chronologies is located in the Aosta area (Figure 9). The orientation of $\sigma_{3}$ axes, however, remains identical for the transcurrent and extensional regimes and we only observe swapping between $\sigma_{1}$ and $\sigma_{2}$.

[41] Extensional tectonics are well expressed throughout the inner western Alpine Arc, from the Simplon pass to the northern tip of the Argentera massif. The main direction of extensional axes is parallel to the structural grain of the Alps
(Figures 4, 6, and 11), as observed in the eastern Alps. Only a minor part of $\sigma_{3}$ axes are oriented perpendicular to the Alpine structures. From a directional viewpoint, the orogenperpendicular extensional axes agree with the current stress regime inferred from seismotectonics. However, it could also be locally related to older extensional structures induced by the relative uplift of large Alpine units, such as the extensional reactivation of the Basal Penninic Thrust behind the Pelvoux massif [Sue and Tricart, 1999], ductile to brittle E-W extension along the western flank of the Gran Paradiso metamorphic dome [Rolland et al., 2000] or large west dipping normal faults on the western side of the Dora Maira and Viso massifs [Schwartz, 2002; Tricart et al., 2004b] during the Miocene. Given the brittle nature of faults, the extension characterized in this paper is postmetamorphic. Faults crosscut (and thus postdate) folds, schistosities and nappe-related ductile structures, even if some extensional movements occurred earlier, at the ductile-brittle transition [Champagnac, 2005, Malusa et al., 2005]. Furthermore, the large diversity of fault plane mineralizations (e.g., calcite, quartz, hematite, talc, amphibole, chlorite), the variety of movement-related lineations (slickenfibers, striations, wear marks) and the aspect of rocks in the vicinity of the fault plane (cataclasite, unconsolidated cataclasite, fault breccias and fault gouges) suggest a very large variation of faulting conditions (temperature, depth, strain rate, fluid pressure and composition) and therefore a long brittle history. Indeed, the fault population observed in the western Alps for this study is the result of a temporal integration of all brittle deformations which occurred in this area, from ductile-brittle transition to the present-day.

\subsection{Geodynamical Interpretations}

[42] Extensional processes are a common feature in evolved orogens and have been widely described [e.g., Dewey, 1988; Molnar and Lyon-Caen, 1988; England and Houseman, 1989; Le Pichon and Chamot-Rooke, 1991; Rey et al., 2001]. Widespread extension in evolved mountain belts has mostly been interpreted in terms of orogenic collapse and/or lateral extrusion (see Rey et al. [2001] and Sue and Tricart [2002] for a review). The onset of such extensional regimes is due to subtle changes in a complex equilibrium, such as a decreasing rate of convergence, increasing erosion rates, temperature-/time-dependent internal strength variations or isostatic reequilibration due to lower crust and/or lithospheric mantle behavior [Avouac and Burov, 1996; Molnar and Lyon-Caen, 1988; Molnar et al., 1993]. In the western Alps, the location of extensional tectonics, the orientation of extensional axes, as well as the lack of current convergence along the Lyon-Torino baseline have led to the proposal that the western Alps are currently in a state of postorogenic collapse [Delacou et al., 2004; Selverstone, 2005; Delacou et al., 2005; Jiménez-Munt et al., 2005], dominated by buoyancy and gravitational forces. This collapse involves an extension perpendicular to the axis of maximal crustal thickness of the belt.

[43] In contrast, extensional deformations described in the eastern Alps [Selverstone, 1988; Frisch et al., 2000] and the central Alps [Mancel and Merle, 1987; Mancktelow, 
1992; Steck and Hunziker, 1994; Nievergelt et al., 1996; Wawrzyniec et al., 2001] have mostly been interpreted in terms of a syncollisional extension, with a main direction of extension oriented along the strike of the belt, and perpendicularly to the main shortening direction. Thus this orogenparallel extension has been interpreted (at least for the eastern Alps) as lateral extrusion toward the east [e.g., Ratschbacher et al., 1991]. As recently supported by numerical modeling [Seyferth and Henk, 2004], lateral extrusion largely prevails in any evolved orogen; a free boundary and overthickened crust are described as favoring factors, however, the plate convergence remains the principal cause.

[44] In the western Alps, we have suggested that the orogen-parallel extension to be due to lateral extrusion toward the south [Champagnac et al., 2004]. The opening of the Ligurian Sea during the lower and middle Miocene [Carminati et al., 1998; Rollet et al., 2002; Rosenbaum et $a l ., 2002]$ would act as a free boundary for the development of a large-scale lateral extrusion system. However, the transition between the inner extensional zones of the Alps and the Ligurian Basin is not straightforward: the southernmost part of the Alpine belt is bent into a tight arc, with the presence of the Argentera massif and Ligurian Alps. Nevertheless, as suggested by fission tracks studies, exhumation of the southern part of the west Alpine belt started in the late Miocene, with a rapid uplift since the Pliocene [BigotCormier et al., 2000; Foeken et al., 2003]. This uplift started at a time, when the Ligurian Sea stopped opening (upper Miocene). Therefore the lower Miocene Alpine arc must have probably been less pronounced than it appears today [Collombet et al., 2002].

[45] In the eastern Alps, lateral extrusion is accommodated by a set of large conjugate dextral and sinistral faults. In the western Alps, large and long-lived dextral transcurrent faults are observed all along the belt, [e.g., Ménard, 1988], but only very few sinistral faults are known (exceptions are the Ospizio Sottile fault [Bistacchi et al., 2000] and some faults within the Schistes Lustrés of the Queyras Ubaye area [Tricart et al., 2004a]). Many authors consider this curved and predominantly dextral strike slip to be due to the counterclockwise rotation of the northern tip of the Apulian indenter [Anderson and Jackson, 1987; Ménard, 1988; Vialon et al., 1989; Collombet et al., 2002]. The scarcity of sinistral faults nicely fits with this interpretation.

[46] We propose that the inner western Alps suffered a large-scale lateral extrusion toward the opening of the Ligurian Sea, at least during lower to middle Miocene. This lateral extrusion would be the origin of the main part of the brittle west Alpine tectonics. A significant part of the extension, however differs significantly from the expected orogen-parallel orientation: orogen-perpendicular or oblique extensional axes are in fact observed all around the belt. An important part of this extension is located in the hanging wall of large normal faults, and occurs under ductile-brittle transition and brittle conditions. This extension is induced by the up-doming and exhumation of basement nappes. It has been observed close to the Simplon pass [Mancktelow, 1992], in the vicinity of the Gran Paradiso and Ambin
Dôme [Rolland et al., 2000; Ganne et al., 2004], and in the Viso and Dora Maira massifs [Schwartz, 2002; Tricart et al., 2004b]. Extension has also been observed in the hanging wall of the reactivated BPT, in the Briançonnais area [Sue and Tricart, 2003] and on the eastern side of the Mont Blanc massif [Seward and Mancktelow, 1994; Aillères et al., 1995; Cannic et al., 1999]. Because of the dynamics of such relative uplift, this extensional tectonics is intimately linked to large-scale compressional deformation ("deep indenter" [e.g., Schwartz, 2002]). The orogen-parallel extrusion described above is also the consequence of shortening within the limits of the Alpine orogeny. Therefore Miocene extensional tectonics can result of the interaction between a major Alpine-scale orogen-parallel extension and a regional-scale "dome-related" extension. This could explain the local multitrend extension (low $\Phi$ ratio) observed.

[47] In summary, our preferred interpretation of the observed extension patterns is shown in Figure 14 which presents three snapshots of Neogene Alpine history: Figure 14a presents the main stage of orogen-parallel extension, directly related to extrusion toward the Ligurian basin, under brittle conditions. This stage predates the end of the Ligurian Basin opening, at 11 Ma [e.g., Rollet et al., 2002]. The external zones still undergo compressive deformation. Figure $14 \mathrm{~b}$ presents the state of stress and strain after the end of the Ligurian Basin opening. Some orogen-parallel extension could be still active. This upper Miocene -Pliocene stage is characterized by rapid uplift of the External Crystalline massif, thrusting in the Jura and Dignes nappe systems as well as dextral motion all along the belt. Figure $14 \mathrm{c}$ presents the orogen-perpendicular extension related to recent paleostress field [Sue and Tricart, 2003; this study] and current strain and stress fields (seismotectonics inversions [Delacou et al., 2004]). The orientation of extensional axes is perpendicular to the belt, whereas compression is limited to a few localities in the external zone. Rotation of the Apulian microplate continue until the present-day [e.g., Anderson and Jackson, 1987; Serpelloni et al., 2005].

\section{Conclusions}

[48] This work fills a gap on brittle deformation analysis left by previous studies. 66 new paleostress tensors have been calculated using the direct inversion method in the Vanoise/Maurienne area (French Alps), between the Aosta Valley [Champagnac et al., 2004] and the Briançonnais area [Sue and Tricart, 2003]. The observed tectonic regime is largely extensional, with $\frac{1}{4}$ of transcurrent paleostress tensors. The main direction of $\sigma_{3}$ axes, for both transcurrent and extensional paleostress tensors, is N-S oriented, parallel to the Alpine structures. A minor part of $\sigma_{3}$ axes is E-W oriented, perpendicular to the Alpine structures. We also performed a synthesis of brittle deformation analyses for the entire arc of the inner western Alps, based on inversion of fault populations. This synthesis highlights the constant presence of an orogen-parallel direction of extension for the complete internal bend of the western Alps: the $\sigma_{3}$ axes are oriented parallel to the Alpine structures, from $\mathrm{N} 065^{\circ}$ in 

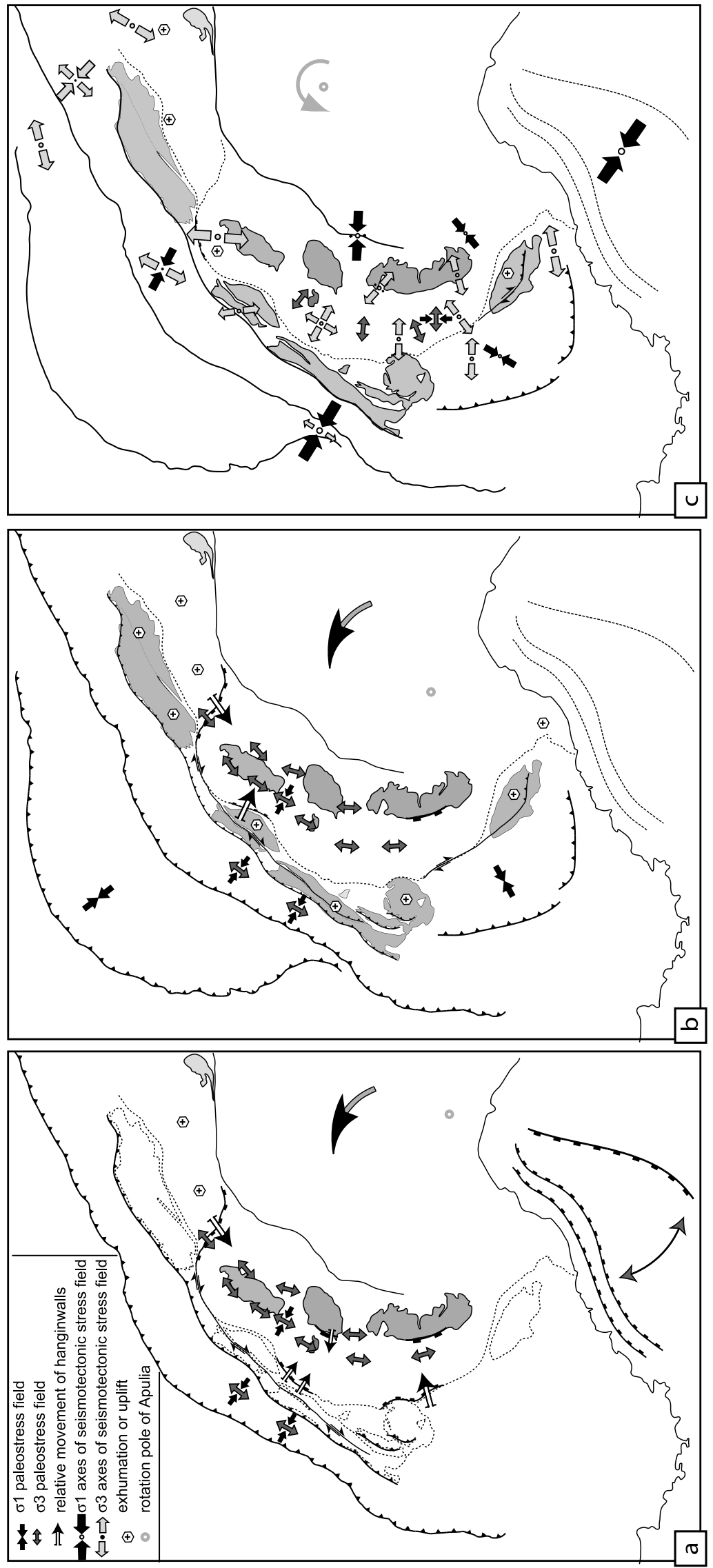

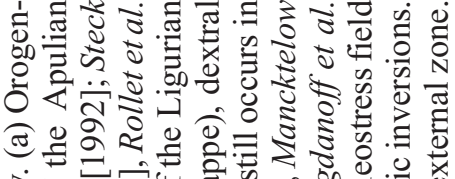

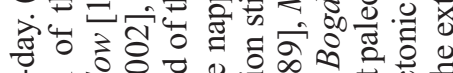

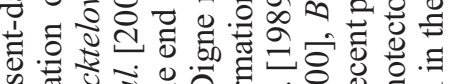

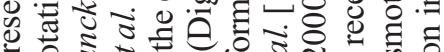

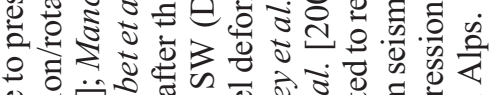

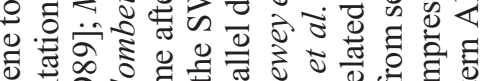

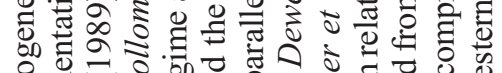
Z.

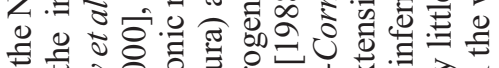

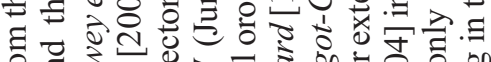

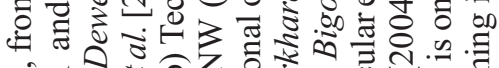

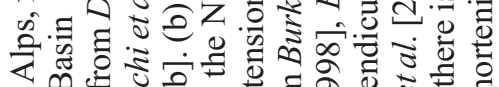

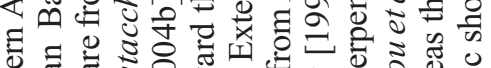

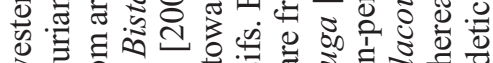
उ.

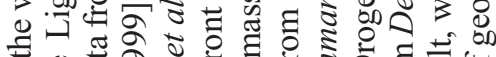

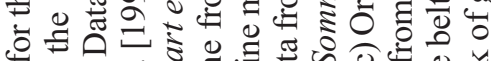

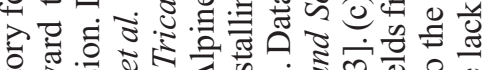

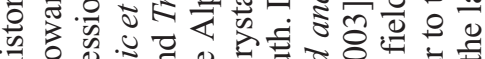

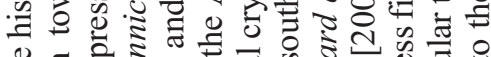

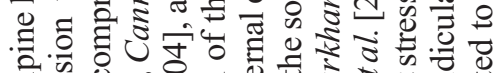

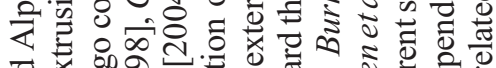
च ख

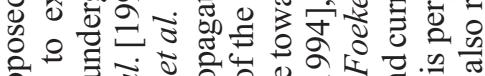

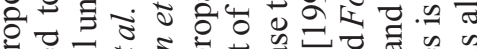

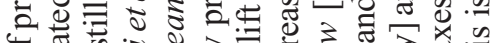

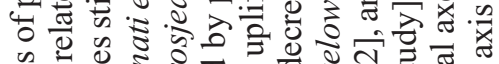

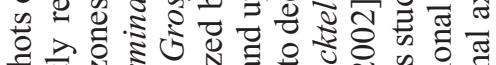
䓎

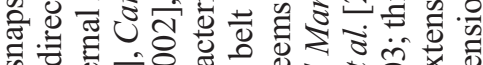

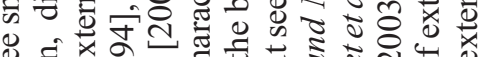

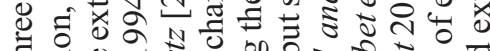
E. 诺 0

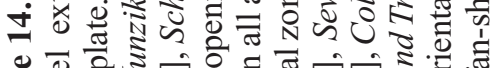

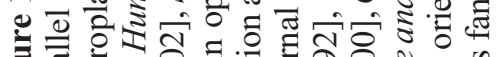

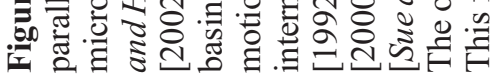


the Simplon and Valais area (Swiss Alps) to N-S in the Vanoise massif, and to NW-SW southward, in the Briançon area. We consider this extension to be related to an extrusion phenomenon toward the south during the Apulian indentation. The opening of the Ligurian Sea during lower to middle Miocene provided a free boundary, which should have promoted this extrusion.

[49] A minor group of $\sigma_{3}$ axes is oriented perpendicularly or obliquely to the Alpine structural trend. This direction of extension becomes more important toward the south, and is prominent in the Briançon area. The origin of this extension seems to be induced by uplift or updoming of External or Internal Crystalline massifs, and/or related to the current postorogenic state of stress [Delacou et al., 2004; Selverstone, 2005; Delacou et al., 2005; Jiménez-Munt et al., 2005].
[50] Last, but not least, a transcurrent regime predates the extension in the Valais area, and postdates it in the other parts of the belt. The $\sigma_{3}$ axes related to the transcurrent paleostress tensors are consistent with $\sigma_{3}$ axes related to extensional paleostress tensors. Stress axes permutations induced by local or regional perturbations and counterclockwise rotational tectonics of the Apulian Microplate explain transcurrent tectonics in the inner western Alps, which is blended with extensional tectonics in the belt.

[51] Acknowledgments. This work was supported by the Neuchâtel and Grenoble (UJF) universities and by the Swiss National Science Fund (grant 21-61684.00, 200020-101625/1, and PBNE2-106764). Authors thank the Associate Editor, Giulio Viola, and an anonymous reviewer for their constructive comments. We are grateful to Tehani Pestalozzi for English corrections of the manuscript.

\section{References}

Aillères, L., J. Bertrand, J. Macaudière, and M. Champenois (1995), New structural data from the "Zone Houillere Brianconnaise" (French Alps), neoalpine tectonics and consequences for the interpretation of the Pennine Front., $C . R$ Acad. Sci., 321, 247-254.

Albarello, D., E. Mantovani, D. Babbucci, and C. Tamburelli (1995), Africa-Eurasia kinematics: Main constraints and uncertainties, Tectonophysics, 243, 25-36.

Anderson, H., and J. Jackson (1987), Active tectonics in the Adriatic region, Geophys. J. R. Astron. Soc., 91, 937-983.

Angelier, J. (1979), Determination of the mean principal direction of stress for a given fault population, Tectonophysics, 56, 17-26.

Angelier, J. (1990), Inversion of field data in fault tectonics to obtain the regional stress: A new rapid direct inversion method by analytical means, Geophys. J. Int., 103, 363-376.

Avouac, J. P., and E. B. Burov (1996), Erosion as a driving mechanism of intracontinental mountain growth, J. Geophys. Res., 101, 17,747-17,769.

Ballèvre, M., Y. Lagabrielle, and O. Merle (1990), Tertiary ductile normal faulting as a consequence of lithospheric stacking in the western Alps, Mem. Soc. Geol. Fr., 156, 27-236.

Baroux, E., N. Béthoux, and O. Bellier (2001), Analyses of the stress field in southeastern France from earthquake focal mechanisms, Geophys. J. Int., 145, 336-348.

Béthoux, N., M. Cattaneo, P. Y. Delpech, C. Eva, and J.-P. Réault (1988), Mécanismes au foyer de séismes en mer Ligure et dans le sud des Alpes occidentales: Résultats et interprétation, $C . R$. Acad. Sci., 307, 71-77.

Bigot-Cormier, F., G. Poupeau, and M. Sosson (2000), Déudations différentielles du massif Cristallin Externe alpin de l'Argentera (Sud-Est de la France) répérés par thermochronologie traces de fission (apatites, zircons): Differential denudations of the Argentera Alpine external crystalline massif (SE France) revealed by fission track thermochronology (zircons, apatites), C. R. Acad. Sci., 330, $363-370$.

Bistacchi, A., E. Eva, M. Massironi, and S. Solarino (2000), Miocene to Present kinematics of the NWAlps: Evidences from remote sensing, structura analysis, seismotectonics and thermochronology, J. Geodyn., 30, 205-228.

Bogdanoff, S., A. Michard, M. Mansour, and G. Poupeau (2000), Apatite fission track analysis in the Argentera massif: Evidence of contrasting denudation rates in the External Crystalline massifs of the western Alps, Terra Nova, 12, 117-125.
Bott, M. H. (1959), The mechanism of oblique slip faulting, Geol. Mag., 96, 109-117.

Brouwer, F. M., D. M. A. van de Zedde, M. J. R. Wortel, and R. L. M. Vissers (2004), Late-orogenic heating during exhumation: Alpine PTt trajectories and thermomechanical models, Earth Planet. Sci. Lett., 220, 185-199.

Burkhard, M. (1988), L'helvétique de la bordure occidentale du massif de l'Aar (évolution tectonique et métamorphique), Eclogae Geol. Helv., 81, $63-$ 114.

Burkhard, M., and A. Sommaruga (1998), Evolution of the western Swiss Molasse basin: Structural relations with the Alps and the Jura belt, in Cenozoic Foreland Basins of Western Europe, edited by A. Mascles et al., Geol. Soc. Spec. Publ., 134, 279-298.

Calais, E., J. M. Nocquet, F. Jouanne, and M. Tardy (2002), Current strain regime in the western Alps from continuous Global Positioning System measurements, 1996-2001, Geology, 30, 651-654.

Cannic, S., J.-L. Mugnier, and J.-M. Lardeaux (1999), Neogene extension in the western Alps, Mem. Sci. Geol. Padova, 51, $33-45$.

Carminati, E., M. J. R. Wortel, P. T. Meijer, and R. Sabadini (1998), The two-stage opening of the western-central Mediterranean basins: A forward modeling test to a new evolutionary model, Earth Planet. Sci. Lett., 160, 667-679.

Carraro, F., G. Guibaudo, M. Giardino, and A. Perotto (1994), Intense deformazioni in depositi fluvioglacustri olocenici nella media valle d'Aosta, Atti Tic. Sci. Terra, 1, 123-136.

Champagnac, J. D. (2005), Tectonique cassante de l'arc interne des Alpes occidentales; implications geodynamiques, Ph.D. thesis, 222 pp., Neuchâtel and J. Fourier Grenoble 1 Univ., Grenoble, France.

Champagnac, J. D., C. Sue, B. Delacou, and M. Burkhard (2003), Brittle orogen-parallel extension in the internal zones of the Swiss Alps (south Valais), Eclogae Geol. Helv., 96, 325-338.

Champagnac, J. D., C. Sue, B. Delacou, and M. Burkhard (2004), Brittle deformation in the inner northwestern Alps: From early orogen-parallel extrusion to late orogen-perpendicular collapse, Terra Nova, 16, 232-242.

Collombet, M., J. C. Thomas, A. Chauvin, P. Tricart, J. P. Bouillin, and J. P. Gratier (2002), Counterclockwise rotation of the western Alps since the Oligocene: New insights from paleomagnetic data, Tectonics, 21(4), 1032, doi:10.1029/ 2001 TC901016.

Deichmann, N., and L. Rybach (1989), Earthquakes and temperatures in the lower crust below the northern Alpine Foreland of Switzerland, in Properties and Processes of Earth's Lower Crust, Geophys. Monogr. Ser., vol. 51, edited by R. F. Mereu, S. Mueller, and D. M. Fountain, pp. 197-213, AGU, Washington, D. C.

Delacou, B. (2005), Tectonique et gédynamique actuelle de l'arc alpin: Approche sismotectonique et modéisation numéique, Ph.D. thesis, $249 \mathrm{pp}$ Neuchâtel and Sophia Antipolis Univ., Nice, France.

Delacou, B., C. Sue, J. D. Champagnac, and M. Burkhard (2004), Present-day geodynamics in the bend of the western and central Alps as constrained by earthquake analysis, Geophys. J. Int., 158, 753-774.

Delacou, B., C. Sue, J. D. Champagnac, and M. Burkhard (2005), Origin of the current stress field in the western/central Alps: Role of gravitational reequilibration constrained by numerical modelling, in Deformation, Rheology and Tectonics: From Minerals to the Lithosphere, edited by D. Gapais et al., Geol. Soc. Spec. Publ., 243, $295-310$.

DeMets, C., R. G. Gordon, D. F. Argus, and S. Stein (1994), Effect of recent revisions to the geomagnetic reversal time scale on estimates of current plate motions, Geophys. Res. Lett., 21, $2191-$ 2194.

Desmons, J., J. Aprahamian, R. Compagnoni, L. Cortesogno, M. Frey, L. Gaggero, G. Dallagiovanna, S. Seno, and L. Radelli (1999), Alpine metamorphism of the western Alps: Middle to high P/T metamorphism. Schweiz. Mineral. Petrogr. Mitt., 79, 89-110.

Dewey, J. F. (1988), Extensional collapse of orogens, Tectonics, 7, 1123-1139.

Dewey, J. F., M. L. Helman, E. Turco, D. W. H. Hutton, and S. D. Knott (1989), Kinematics of the western Mediterranean., in Alpine Tectonics, edited by M. Coward et al., Geol. Soc. Spec. Publ., 45, 265-283.

Diamond, L. W. (1990), Fluid inclusion evidence for PV-T-X evolution of hydrothermal solutions in LateAlpine gold-quartz veins at Brusson, Val d'Ayas, northwest Italian Alps., Am. J. Sci., 290, 912-958.

England, P., and G. Houseman (1989), Extension during active convergence, with application to the $\mathrm{Ti}$ betan Plateau, J. Geophys. Res., 94, 17,56117,579 .

Escher, A., and C. Beaumont (1997), Formation, burial and exhumation of basement nappes at crustal scale: A geometric model based on the western Swiss-Italian Alps, J. Struct. Geol., 19, 955-974.

Eva, E., S. Pastore, and N. Deichmann (1998), Evidence for ongoing extensional deformation in the 
western Swiss Alps and thrust-faulting in the southwestern Alpine foreland, J. Geodyn., 26 , $27-43$.

Foeken, J. P. T., T. J. Dunai, G. Bertotti, and P. A. M. Andriessen (2003), Late Miocene to present exhumation in the Ligurian Alps (southwes Alps) with evidence for accelerated denudation during the Messinian salinity crisis, Geology, $31,797-800$

Frisch, W., I. Dunk1, and J. Kuhlemann (2000), Postcollisional orogen-parallel large-scale extension in the eastern Alps, Tectonophysics, 327, 239-265.

Ganne, J., J.-M. Bertrand, and S. Fudral (2004), Geometry and kinematics of early Alpine nappes in a Brianconnais basement (Ambin massif, western Alps), C. R. Geosci., 336, 1219-1226.

Grosjean, G., C. Sue, and M. Burkhard (2004), Late Neogene brittle extension in the vicinity of the Simplon fault zone, central Alps, Switzerland, Eclogae Geol. Helv., 97, 33-46.

$\mathrm{Hu}$, J. C., and J. Angelier (2004), Stress permutations: Three-dimensional distinct element analysis account for a common phenomenon in brittle tectonics, J. Geophys. Res., 109, B09403, doi:10.1029/2003JB002616.

Jiménez-Munt, I., D. Garcia-Castellanos, A. M Negredo, and J. P. Platt (2005), Gravitational and tectonic forces controlling postcollisional deformation and the present-day stress field of the Alps: Constraints from numerical modeling, Tectonics, 24, TC5009, doi:10.1029/2004TC001754

Kastrup, U., M. L. Zoback, N. Deichmann, K. Evans, and D. Giardini (2004), Stress field variations in the Swiss Alps and the northern Alpine foreland derived from inversion of fault plane solutions, J. Geophys. Res., 109, B09403, doi:10.1029/ 2003JB002616

Lemoine, M., et al. (1986), The continental margin of the Mesozoic Tethys in the western Alps, Mar. Pet Geol., 3, 179-199.

Le Pichon, X., and N. Chamot-Rooke (1991), Extension of continental crust, in Controversies in Modern Geology: Evolution of Geological Theories in Sedimentology, Earth History and Tectonics, edited by D. W. Müller, J. A. McKenzie, and H. J. Weissert, pp. 313-338, Elsevier, New York.

Lickorish, W. H., M. Ford, J. Bürgisser, and P. R. Cobbold (2002), Arcuate thrust systems in sandbox experiments: A comparison to the external arcs of the western Alps, Geol. Soc. Am. Bull., 114, 1089 1107.

Malusa, M. G., R. Polino, M. Zattin, G. Bigazzi, S. Martin, and F. Piana (2005), Miocene to Present differential exhumation in the western Alps: Insights from fission tracks thermochronology, Tectonics, 24 , TC3004, doi:10.1029/2004TC001782.

Mancel, P., and O. Merle (1987), Kinematics of the northern part of the Simplon line (central Alps), Tectonophysics, 135, 265-275.

Mancktelow, N. S. (1992), Neogene lateral extension during convergence in the central Alps: Evidence interrelated faulting and backfolding around the Simplon pass (Switzerland), Tectonophysics, 215, $295-317$.

Ménard, G. (1988), Structure et cinéatique d'une chaîne de collision: Les Alpes occidentales et centrales, thèse de Doctorat d'Etat, 278 pp., Univ. Joseph Fourier, Grenoble, France.

Molnar, P., and H. Lyon-Caen (1988), Some simple physical aspects of the support, structure, and evolution of mountain belts, Geol. Soc. Am. Bull., 218 , 179-207.

Molnar, P., P. England, and J. Martinod (1993), Mantle dynamics, the uplift of the Tibetan Plateau and the Indian monsoon, Rev. Geophys., 31, 123-153.

Nievergelt, P., M. Liniger, N. Froitzheim, and R. F. Maehlmann (1996), Early to mid Tertiary crustal extension in the central Alps: The Turba mylonite zone (eastern Switzerland), Tectonics, 15, 329340

Nocquet, J. M., and E. Calais (2004), Geodetic measurements of crustal deformation in the western
Mediterranean and Europe, Pure Appl. Geophys, $161,661-681$

Oldow, J. S., L. Ferranti, D. S. Lewis, J. K. Campbell, B. D'Argenio, R. Catalano, G. Pappone, L. Carmignani, P. Conti, and C. L. V. Aiken (2002), Active fragmentation of Adria, the north African promontory, central Mediterranean orogen, Geology, 30, 779-782.

Pavoni, N. (1980), Comparison of focal mechanisms of earthquakes and faulting in the Helvetic zone of the central Valais, Swiss Alps, Eclogae Geol. Helv., 73 , $551-558$.

Pfiffner, O. A., S. Ellis, and C. Beaumont (2000), Collision tectonics in the Swiss Alps: Insight from geodynamic modeling, Tectonics, 19, 1065-1094.

Ratschbacher, L., W. Frisch, H.-G. Linzer, and O. Merle (1991), Lateral extrusion in the eastern Alps: 2, Structural analysis, Tectonics, 10, 257-271.

Rey, P., O. Vanderhaeghe, and C. Teyssier (2001), Gravitational collapse of the continental crust: Definition, regimes and modes, Tectonophysics, 342 , $435-449$.

Robin, P.-Y. F., and E. C. Jowett (1986), Computerized density contouring and statistical evaluation of orientation data using counting circles and continuous weighting functions., Tectonophysics, 121, 207-233.

Rolland, Y., J. M. Lardeaux, S. Guillot, and C. Nicollet (2000), Syn-convergence extension, vertical pinching and contrasted metamorphic units on the western edge of the Gran Paradise massif (French-Italian Alps), Geodyn. Acta, 13, 133-148.

Rollet, N., J. Deverchere, M. O. Beslier, P. Guennoc, J. P. Rehault, M. Sosson, and C. Truffert (2002), Back arc extension, tectonic inheritance, and volcanism in the Ligurian Sea, western Mediterranean, Tectonics, 2l(3), 1015, doi:10.1029/2001TC900027.

Rosenbaum, G., G. S. Lister, and C. Duboz (2002), Reconstruction of the tectonic evolution of the western Mediterranean since the Oligocene, J. Virtual Explor., 8, 107-130.

Schmid, S. M., and E. Kissling (2000), The arc of the western Alps in the light of geophysical data on deep crustal structure, Tectonics, 19, 62-85.

Schwartz, S. (2002), La zone piéontaise des Alpes occidentales: Un paléo-complexe de subduction. Arguments métamorphiques, géochronologiques et structuraux, Ph.D. thesis, $341 \mathrm{pp}$, Univ. Claude Bernard, Lyons, France.

Selverstone, J. (2005), Are the Alps collapsing?, Annu Rev. Earth Planet. Sci., 33, 1-20.

Selverstone, S. M. (1988), Evidences for east-wes crustal extension in the eastern Alps: Implication for the unroofing history of the Tauern window, Tectonics, 7, 87-105.

Serpelloni, E., M. Anzidei, P. Baldi, G. Casual, and A. Galvani (2005), Crustal velocity and strain-rate fields in Italy and surrounding regions: New results from the analysis of permanent and non-permanen GPS networks, Geophys. J. Int., 161, 861-880, doi:1111/j.1365-264X.2005.02618.x.

Seward, D., and N. S. Mancktelow (1994), Neogene kinematics of the central and western Alps: Evi dence from fission-track dating, Geology, 22, $803-806$.

Seyferth, M., and A. Henk (2004), Syn-convergent exhumation and lateral extrusion in continental collision zones: Insights from three-dimensional numerical models, Tectonophysics, 382, 1-29.

Smith, W. H. F., and P. Wessel (1990), Gridding with continuous curvature splines in tension, Geophysics, $55,293-305$.

Sperner, B., R. Ott, and L. Ratschbacher (1993), Faultstriae analysis: A turbo Pascal program package for graphical presentation and reduced stress-tensor calculation, Comput. Geosci., 19, $1361-1388$.

Steck, A., and J. Hunziker (1994), The tertiary structure and thermal evolution of the central Alps: Compressional and extensional structures in an orogenic belt, Tectonophysics, 238, 229-254.

Steck, A., J. L. Epard, A. Escher, R. Marchant, and F. Masson (1997), Geological interpretation of the seismic profiles through western Switzerland: Rawil (W1), Val d'Annivier (W2), Matterta (W3), Zmutt-Zermatt-Findelen (W4), and Val de Bagnes (W5), in Results of NRP 20: Deep Structure of the Swiss Alps, edited by O. A. Pfiffner et al., Springer, New York.

Sue, C., and P. Tricart (1999), Late Alpine brittle extension above the Frontal Pennine Thrust neat Briancon, western Alps, Eclogae Geol. Helv., 92, $171-181$.

Sue, C., and P. Tricart (2002), Widespread post-nappe normal faulting in the internal western Alps: A new constrain on arc dynamic, J. Geol. Soc. London, $159,61-70$.

Sue, C., and P. Tricart (2003), Neogene to ongoing normal faulting in the inner western Alps: A major evolution of the late alpine tectonics, Tectonics, 22(5), 1050, doi:10.1029/2002TC001426.

Sue, C., F. Thouvenot, J. Frechet, and P. Tricart (1999), Widespread extension in the core of the western Alps revealed by earthquake analysis, $J$. Geophys Res., 104, 25,611-25,622.

Sue, C., J. Martinod, P. Tricart, F. Thouvenot, J. F. Gamond, J. Frechet, D. Marinier, J. P. Glot, and J. R. Grasso (2000), Active deformation in the inner western Alps inferred from comparison between 1972-classical and 1996-GPS geodetic surveys, Tectonophysics, 320, 17-29.

Tricart, P. (1984), From passive margin to continental collision: A tectonic scenario for the western Alps, Am. J. Sci., 284, $97-120$

Tricart, P., S. Amaudric du Chaffaut, C. Ayoub, M. Ballère, R. Caby, C. Gout, Y. Lagabrielle, D. Leblanc, O. P. P. Le Mer, and P. Saby (2004a), Carte géologique de la France au 1/50000eme, feuille 848 Aiguille-Col Sain Martin, Bur. de Rec. Geol. et Min., Orléans, France.

Tricart, P., S. Schwartz, C. Sue, and J.-M Lardeaux (2004b), Evidence of synextension tilting and doming during final exhumation from analysis of multistage faults (Queyras Schistes lustrés, western Alps), J. Struct. Geol, 26, 1633-1645.

Vialon, P., P. Rochette, and G. Ménard (1989), Indentation and rotation in the Alpine arc., in Alpine Tectonics, edited by M. Coward et al., Geol. Soc. Spec. Publ., 45, 329-338.

Villemin, T., and H. Charlesworth (1992), Stress: A program to analyze data from Striated fault. International Union of Geological Science Commission on Tectonics, Shorts Course on Some Applications of IBM-PC Compatible Micro-computer in Structural Geology, Cent. Am. Sch. of Geol., Univ. de Costa Rica, San Jose.

Wallace, R. E. (1951), Geometry of shearing stress and relation to faulting, J. Geol., 59, 118-130.

Wawrzyniec, T. F., J. Selverstone, and G. J. Axen (2001), Style of footwall uplift along the Simplon and Brenner normal fault system, central and eastern Alps, Tectonics, 20, 748-770.

Yamaji, A. (2000), The multiple inverse method: A new technique to separate stresses from heterogeneous fault-slip data, J. Struct. Geol., 22, $441-$

Zwingmann, H., and N. Mancktelow (2004), Timing of Alpine fault gouges, Earth Planet. Sci. Lett., 223 $415-425$.

C. Allanic, M. Burkhard, B. Delacou, and C. Sue, Geological Institute, University of Neuchâtel, Rue Argand 11, CP2, CH-2000 Neuchâtel, Switzerland.

J. D. Champagnac, Department of Geological Sciences/CIRES, Benson Earth Sciences Building, University of Colorado, Boulder, CO 80309, USA. (champagn@colorado.edu)

P. Tricart, Laboratoire de Géodynamique des Chaînes Alpines (LGCA), Maison des Géosciences, Université de Grenoble, F-38400 St Martin D’Hères, France. 